Portland State University

PDXScholar

Spring 7-21-2015

\title{
A Multivariate Modeling Approach for Generating Ensemble Climatology Forcing for Hydrologic Applications
}

Sepideh Khajehei

Portland State University

Follow this and additional works at: https://pdxscholar.library.pdx.edu/open_access_etds

Part of the Civil and Environmental Engineering Commons, and the Hydrology Commons Let us know how access to this document benefits you.

\section{Recommended Citation}

Khajehei, Sepideh, "A Multivariate Modeling Approach for Generating Ensemble Climatology Forcing for Hydrologic Applications" (2015). Dissertations and Theses. Paper 2403.

https://doi.org/10.15760/etd.2400

This Thesis is brought to you for free and open access. It has been accepted for inclusion in Dissertations and Theses by an authorized administrator of PDXScholar. For more information, please contact pdxscholar@pdx.edu. 
A Multivariate Modeling Approach for Generating Ensemble Climatology Forcing for Hydrologic Applications

\author{
By
}

Sepideh Khajehei

\begin{abstract}
A thesis submitted in partial fulfillment of the requirements for the degree of Master of Science

in

Civil Engineering
\end{abstract}

Thesis Committee:

Hamid Moradkhani, Chair

Heejun Chang

Arun Rana

Portland State University 


\begin{abstract}
Reliability and accuracy of the forcing data plays a vital role in the Hydrological Streamflow Prediction. Reliability of the forcing data leads to accurate predictions and ultimately reduction of uncertainty. Currently, Numerical Weather Prediction (NWP) models are developing ensemble forecasts for various temporal and spatial scales. However, it is proven that the raw products of the NWP models may be biased at the basin scale; unlike model grid scale, depending on the size of the catchment. Due to the large space-time variability of precipitation, bias-correcting the ensemble forecasts has proven to be a challenging task. In recent years, Ensemble Pre-Processing (EPP), a statistical approach, has proven to be helpful in reduction of bias and generation of reliable forecast. The procedure is based on the bivariate probability distribution between observation and single-value precipitation forecasts. In the current work, we have applied and evaluated a Bayesian approach, based on the Copula density functions, to develop an ensemble precipitation forecasts from the conditional distribution of the single-value precipitation. Copula functions are the multivariate joint distribution of univariate marginal distributions and are capable of modeling the joint distribution of two variables with any level of correlation and dependency. The advantage of using Copulas, amongst others, includes its capability of modeling the joint distribution independent of the type of marginal distribution. In the present study, we have evaluated the capability of copula-based functions in EPP and comparison is made against an existing and commonly used procedure for same i.e. meta-Gaussian distribution. Monthly precipitation forecast from Climate Forecast System (CFS) and gridded observation from Parameter-elevation
\end{abstract}


Relationships on Independent Slopes Model (PRISM) have been utilized to create ensemble pre-processed precipitation over three sub-basins in the western USA at 0.5degree spatial resolution. The comparison has been made using both deterministic and probabilistic frameworks of evaluation. Across all the sub-basins and evaluation techniques, copula-based technique shows more reliability and robustness as compared to the meta-Gaussian approach. 
Dedication

To:

My family

for debts I can never repay 


\section{Acknowledgments}

I would like to express the deepest appreciation to my advisor, Dr. Hamid Moradkhani, who has the attitude and the substance of a genius: he continually and convincingly conveyed a spirit of adventure in regards to research and an excitement in regards to teaching. Without his guidance and persistent help, this thesis would not have been possible. I also would like to thank my committee members, Dr. Heejun Chang and Dr. Arun Rana, for their support and for their timely review of my thesis. I greatly appreciate their willingness to serve in my thesis committee. For all the members of my research group, I thank you all for your hard work and positive attitude during all of our collaboration, and providing a great friendly atmosphere in the lab.

I must thank my family for their endless support. Thank you to my parents for their generous support both financially and mentally throughout my academic journey. Special thanks to my Aunt, who has been my guardian all the time and without her financial support, it would not be possible for me to pursue my goals. Lastly, I appreciate my sister for being my best friend, who has provided me with a strong love shield that always protects me from sadness. 
Table of Contents

Abstract

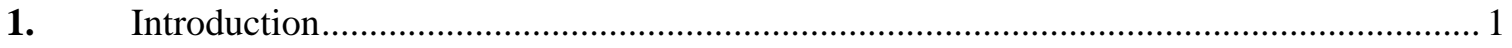

Chapter 2

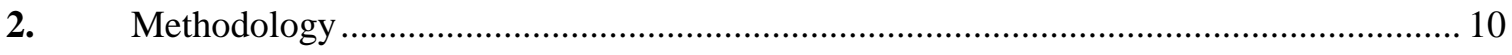

Chapter 3

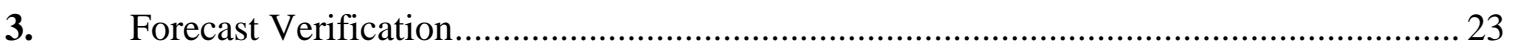

Chapter 4

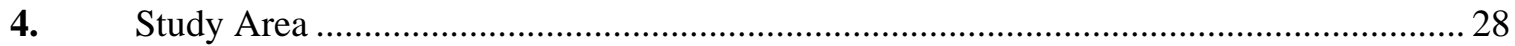

Chapter 5

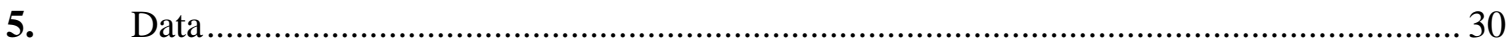

Chapter 6

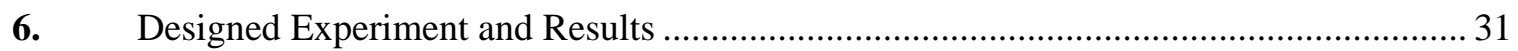

Chapter 7

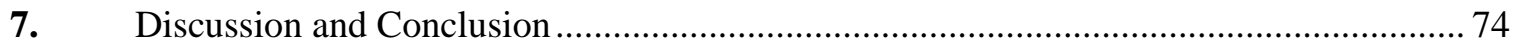

Chapter 8

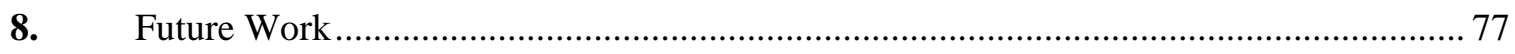

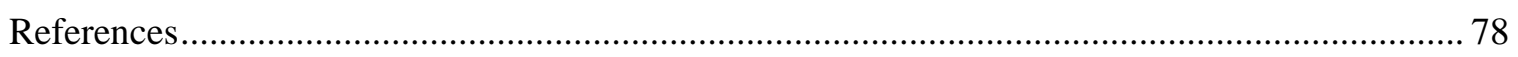




\section{List of Figures}

Figure1 1...

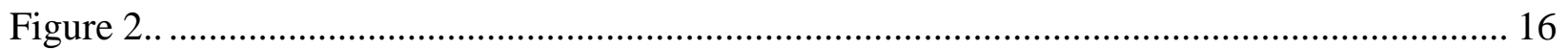

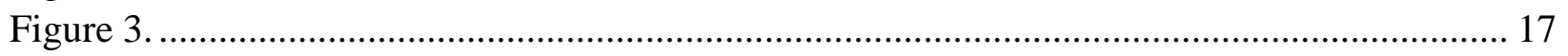

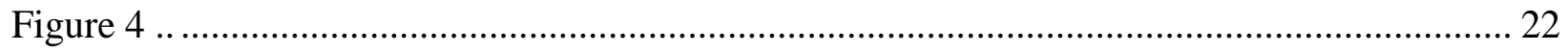

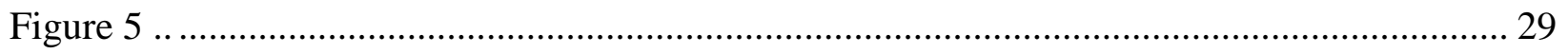

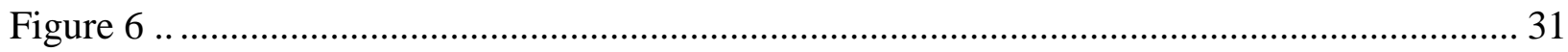

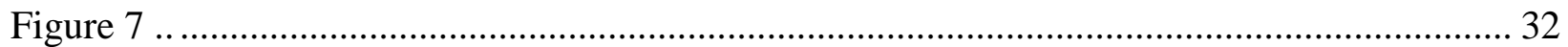

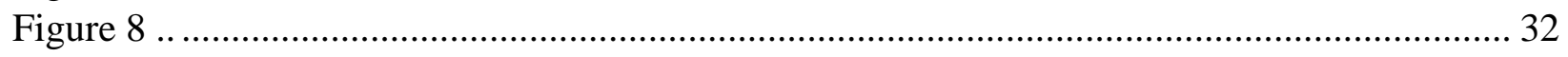

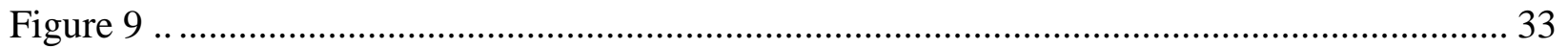

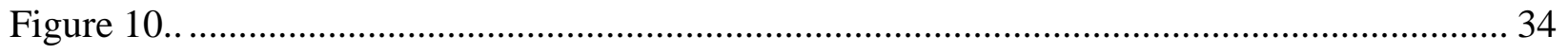

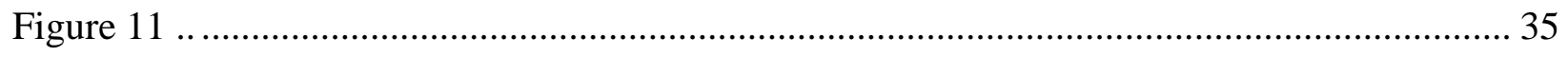

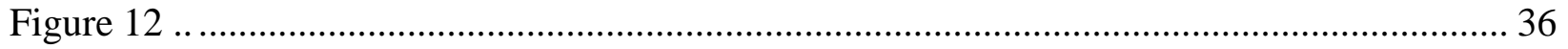

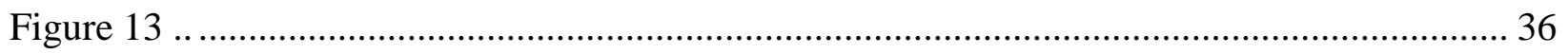

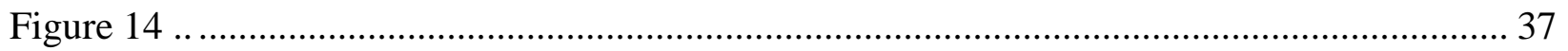

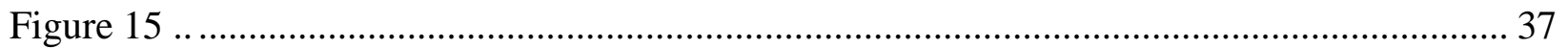

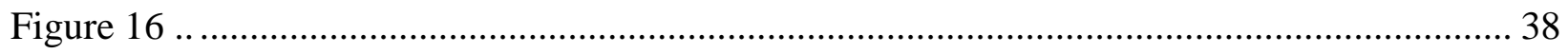

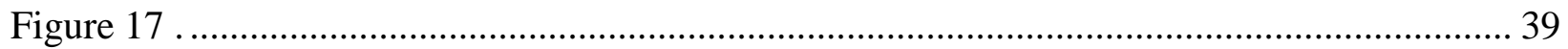

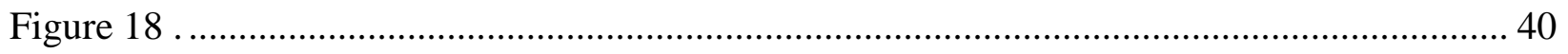

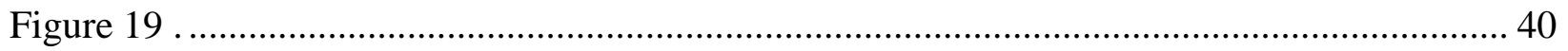

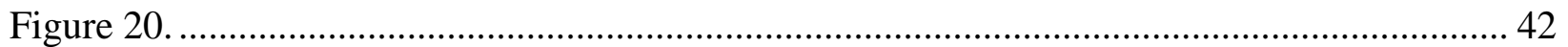

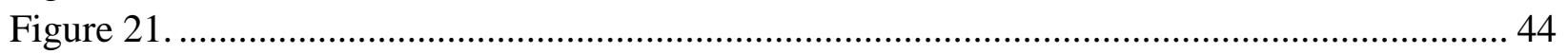

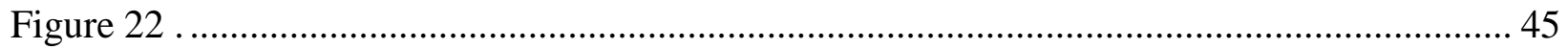

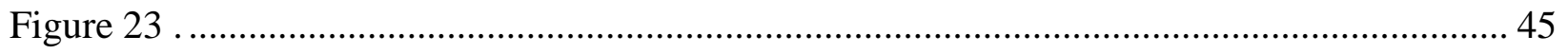

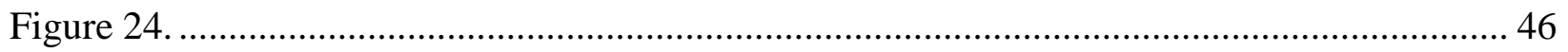

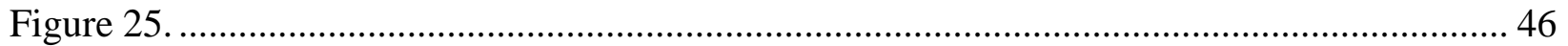

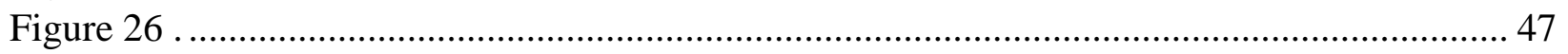

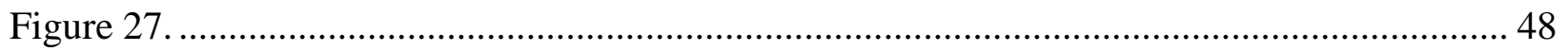

Figure 28 .

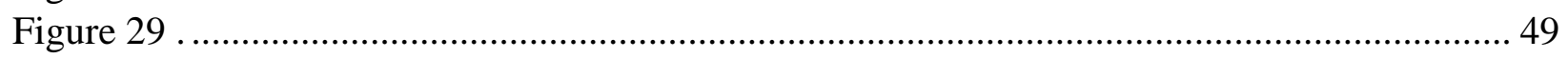

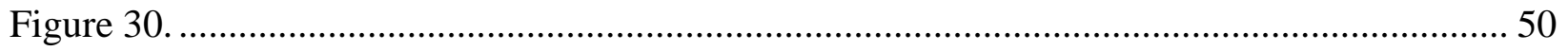

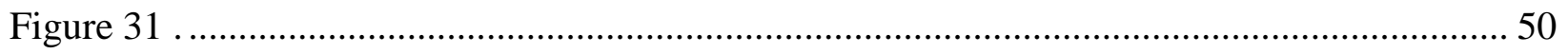

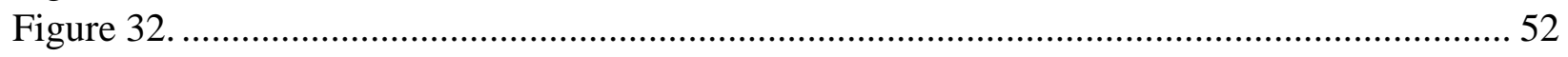

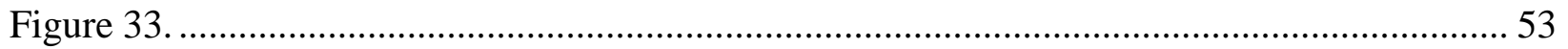

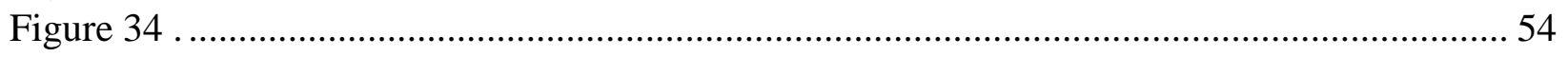

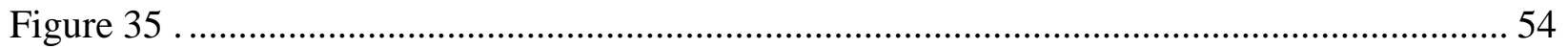

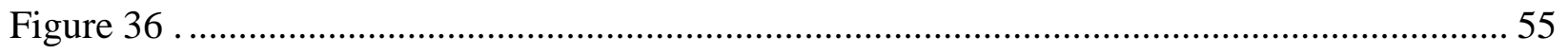

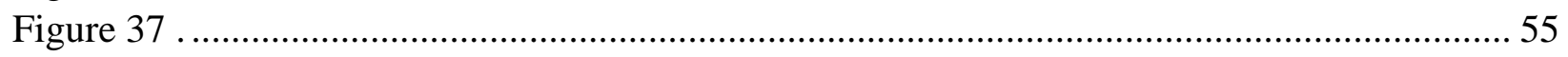

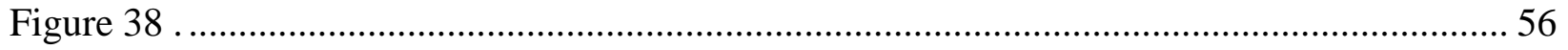

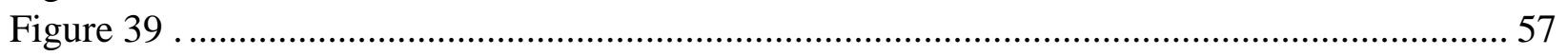




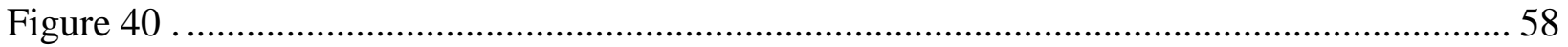

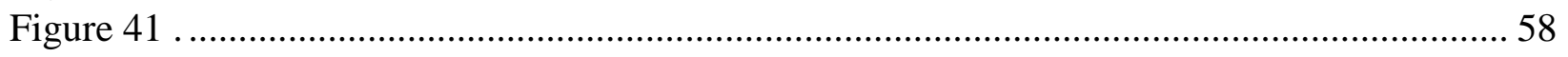

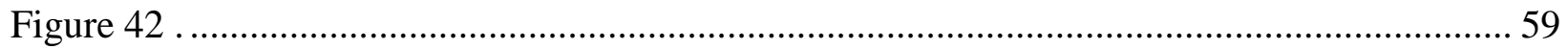

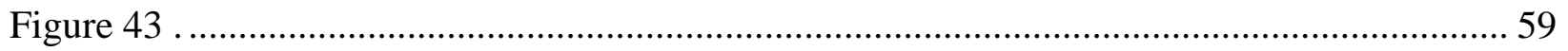

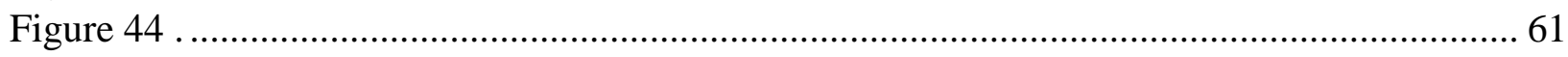

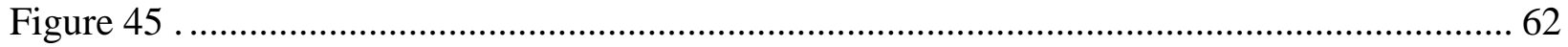

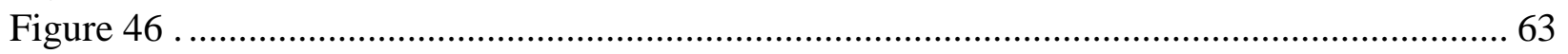

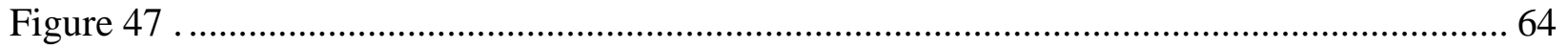

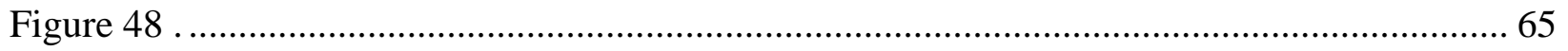

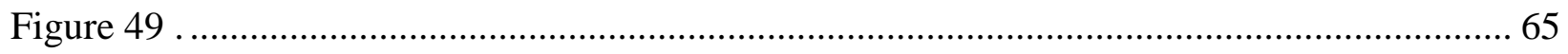

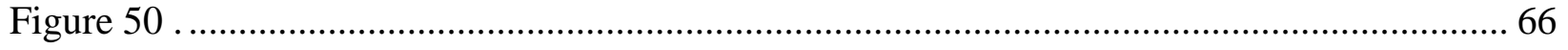

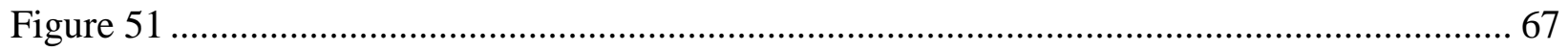

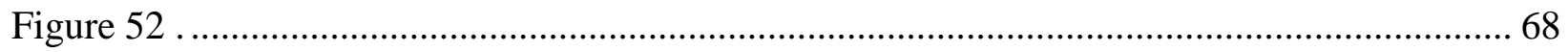

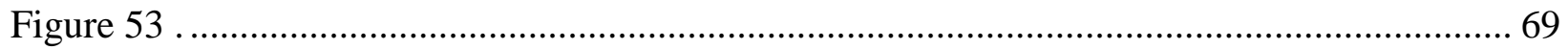

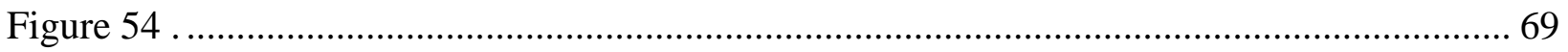

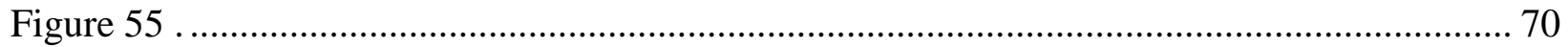

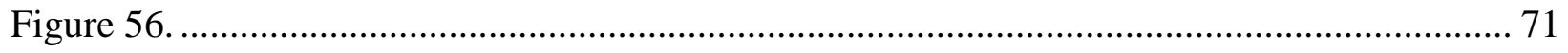

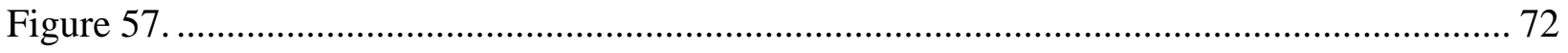

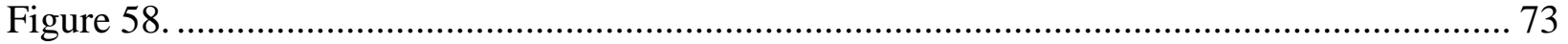


List of Tables

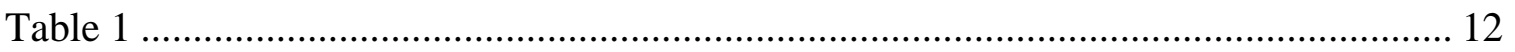

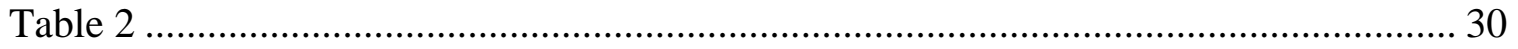




\section{Introduction}

Water related parameters, in various sectors, such as energy demand/supply chain, hydrology, agriculture, fisheries management etc., are essential in decision-making process for the water-management/risk-assessment organizations/agencies. Currently most of the studies related to dam construction, preserving fish species, agricultural management, drought/flood forecasting, amongst others; rely on the products of weather and hydrologic forecast. Weather forecasters are responsible for providing accurate climatological forecasts, which in-turn is affected by uncertainty in the climate models. The hydrologists' responsibility is to deploy information provided by the climate forecasts and generate hydrologic predictions, which are reliable for use in future studies. Hydrologic processes are strongly affected by climatic variables. On the other hand, future climatology itself is not certain owing to various factors, including forecast uncertainty, and usually relies on

the historical behavior of climate data for the forecast of future conditions further introducing uncertainty to hydroclimatology. Therefore, it is desirable/necessary to handle the uncertainty in the climate data itself to reduce the input uncertainty for the hydrologic applications and make them more reliable.

The key factors associated with uncertainty in hydrologic simulations are related to the reliability/uncertainty associated with the forcing data, parameters, initial condition and hydrologic models. Accurate predictions are based on the assumptions of accuracy in each of these components of hydrological forecasting. In the past few years, scientists have introduced various techniques to handle uncertainty in different aspects. Amongst them, 
the statistical procedures have been implemented successfully to reduce the uncertainty associated with each of these elements and ultimately predictions.

Various components of uncertainty have been dealt in literature with different techniques. Firstly, data assimilation is introduced to deal with the uncertainty in the initial and boundary conditions (DeChant and Moradkhani, 2014; Moradkhani et al., 2012; Moradkhani et al., 2005a; Moradkhani and Sorooshian, 2008; Moradkhani et al., 2005b). Further, uncertainty in model structure can be improved by multi-modeling and Ensemble Streamflow Prediction (ESP); which have been developed and improved by utilization of statistical techniques such as Bayesian Model Averaging (DeChant and Moradkhani, 2011; Madadgar and Moradkhani, 2014; Madadgar et al., 2014; Moradkhani et al., 2005b; Najafi and Moradkhani, 2014; Parrish et al., 2012). Whereas, uncertainty in the streamflow predictions is usually dealt with bias correction methods such as quantile mapping, multicriteria regression methods and statistical procedures based on bivariate joint distributions, amongst others (Madadgar et al., 2014; Wood and Schaake, 2008; Zhao et al., 2011). Lastly, uncertainty in climate forcing data can be handled with statistical procedures such as bias correction and downscaling (Li et al., 2010; Sharma et al., 2007; Wood et al., 2004). Recently, techniques for generating ensemble meteorological forecasts are widely practiced in the climate science community (Clark et al., 2004; Robertson et al., 2013; Schaake et al., 2007; Tao et al., 2014; Wu et al., 2011; Zhao et al., 2011). It is proven that using ensemble forecasts helps to address the uncertainty of most likely events (Park et al., 2008; Tao et al., 2014). 
Today, Numerical Weather Prediction (NWP) models serve as main source of data for ensemble forecasts. The models are generating the ensemble forecasts across diverse spatial and temporal ranges. Ensemble forecasts are produced by running the NWP regional and global models with different initial conditions that provides information of the most likely events and uncertainty associated with them. Among the various products, ShortRange Ensemble Forecast (SREF), the Global Ensemble Forecast System (GEFS), and the Climate Forecast System (CFS) are generated by the National Weather Service's (NWS) National Centers for Environmental Prediction (NCEP) models (Park et al., 2008; Tao et al., 2014; Wu et al., 2011).

Despite of recent progress in climate models, forecasts are still subjected to errors in mean and spread (Hamill and Whitaker, 2006; Wu et al., 2011). This could be attributed to initial conditions and model errors, amongst others. These errors/deviations are more significant in climatic variables, such as precipitation, that are affected drastically by change in spatial scale. Even if the variable is free of bias on model scale, it would still be prone to errors on a larger catchment scale (Wu et al., 2011). Thus, it is not advised to utilize the ensemble forecasts directly due to three principal reasons, namely: (1) Lack of accuracy in the ensemble climate forecasts, which effect their applicability in the hydrologic processes, (2) The spread of raw ensemble forecasts might not be trustworthy due to the uncertainty for inconsistency of observations chosen, and (3) The spatial scale is prone to large errors since the global climate models mostly are run over grids that are larger than the basin scale (Rayner et al., 2005; Tao et al., 2014). 
Given above listed problems in ensemble forecasts, there is a demand for generating forecasts that are more reliable on smaller grid or a catchment scale. Different techniques has been applied and tested in literature thus far, such as use of statistical methods in calibration of raw climate forecasts (Tao et al., 2014; Wood and Schaake, 2008). On the other hand, calibrating and bias correcting the raw ensemble forecasts itself has proven to be a challenging task for researchers (Hamill and Whitaker, 2006; Wu et al., 2011). Thus, research have focused on generating ensemble meteorological forecasts from single-value forecasts, on the basin scale (Wu et al., 2011).

Owing to the huge demand of statistical techniques and its simplicity over other methods for generation of reliable ensemble forecasts, various approaches have been endorsed and practiced. Bayesian Model Averaging (Raftery et al., 2005) and analog techniques (Hamill et al., 2006) are among the popular methods. Recently, a new statistical technique for Ensemble Pre-Processing (EPP) was developed and used by U.S. National Weather Service River Forecast System (NWSRES), which utilizes the relation between observation and forecasts captures the correlation between the two datasets. This technique has been implemented, revised and improved over diverse spatial and temporal scales and variables (both precipitation and temperature) forecast over the past few years (Demargne et al., 2010; Liu et al., 2013; Robertson et al., 2013; Schaake et al., 2007; Tao et al., 2014; Wood et al., 2004; Wood and Schaake, 2008; Wu et al., 2011). The procedure is termed as Ensemble Post-Processing if the said technique is applied after (-post) generation of forecast. Based on the application of technique (before or after), it is called as PreProcessing in context of hydrology, since it is procedure for preparing input for hydrologic 
model, whereas in context of climatology it is termed as Post-Processing, since it is the processing the output from climate models.

In EPP, the statistical procedure aims to build bivariate joint distribution between the observation and forecast in training period. In the past few years, various techniques have been tested and implemented to achieve the said objective. (Kelly and Krzysztofowicz, 1997) introduced a bivariate meta-Gaussian distribution function, which was based on the normal quantile transform of the two variables according to Gaussian law in the Bayesian Forecasting System (BFS). The above procedure was further employed by (Krzysztofowicz and Herr, 2001) to assess the uncertainty of precipitation data. In comparison to Gaussian model, the above procedure highlights four major aspects: 1) using any form of the marginal distribution for the variables, 2) allowing modeling with prior densities and likelihood functions, 3) presence of dependence parameters, and finally 4) access to analytical form which is simple to evaluate. (Seo et al., 2000) developed a procedure to generate ensemble precipitation space-time series based on (Krzysztofowicz and Herr, 2001) methodology, and the results were encouraging, for small scale watersheds. (Clark and Hay, 2004) employed statistics to downscale the model variables of the global forecast system (GFS), which was known as the medium range forecast system developed in the NWS cooperative network. To reduce the uncertainty in scaletime variability of the meteorological variables, (Clark et al., 2004) developed the Schaake Shuffle procedure for reconstructing the ensemble members in accordance to historical values. (Schaake et al., 2007) described the full procedure based on mixed distribution of two variables and application of transformation that (Kelly and Krzysztofowicz, 1997) 
applied for building the joint distribution between two non-normal variables. This method that have been used by the National Weather Service River Forecasting System (NWSRFS) for developing the ensemble meteorological forecasts as the input to ESP. The procedure is simple and uses fewer parameters. This procedure is widely used by the NWSRFS and Hydrological Ensemble Prediction Experiment (HEPEX), ultimately known as Ensemble Pre-Processing (EPP).

In recent years, (Wu et al., 2011) developed the mixed type meta-Gaussian joint distribution on lines of (Kelly and Krzysztofowicz, 1997) and thus resulting in a procedure that model the precipitation intermittency decisively. It can be compared to the (Schaake et al., 2007) method, which models each of the marginal distribution as a convex of the continuous distribution. (Robertson et al., 2013) used the Bayesian Joint Probability (BJP) developed by (Wang and Robertson, 2011; Wang et al., 2009) for generating the ensemble precipitation forecast on sub-daily scale in Australia. The methodology adopted by them have been described with few parameters, one the challenges in EPP. (Tao et al., 2014) have applied EPP in combination of multi-modeling to generate more reliable forecast from The Observing System Research and Predictability Experiment (THORPEX) Interactive Grand Global Ensemble (TIGGE) products.

The procedures described above require transformation function for both observation and forecasts that do not follow a normal distribution. For instance, (Robertson et al., 2013) used log-sinh transform and (Schaake et al., 2007; Tao et al., 2014; Wu et al., 2011) employed normal quantile transform. These transformation process might affect the accuracy of the estimated probability distribution between the observation and forecast 
(Brown and Seo, 2013; Madadgar and Moradkhani, 2014; Madadgar et al., 2014). The forecast probability distribution in above techniques assumes a multivariate normal distribution, which adheres observation with forecast variables. Furthermore, usage of normal joint distribution requires transformation of non-Gaussian distributive variable into normal space. The transformation may also affect accuracy of the estimated probability distribution (Brown and Seo, 2013; Madadgar and Moradkhani, 2014). To overcome the said problem, (Brown and Seo, 2013) introduced a new approach based on non-parametric probability distribution. They employed discrete set of thresholds for observed variable in his non-parametric probability distribution. (Madadgar et al., 2014) demonstrated that the non-parametric probability distribution is highly dependent on the number of thresholds of observed variables. Consequently, it would be beneficial to develop a procedure that can capture the uncertainty. In a way that there would be no need for distribution transformation and thus evolve an effective probability distribution.

(Sklar, 1959) introduced the construction of multivariate distributions in the notion of Copulas. They are useful in capturing the drawbacks of most of the multivariate distributions e.g. bivariate Pareto, bivariate gamma etc. Copula has the capability to draw the joint distribution that is self-sufficient of the marginal distribution (Favre et al., 2004; Joe, 1997) .It is widely well-known in the financial studies (Cherubini et al., 2004). Currently, copulas have been used in the hydrological domains as well, (Bárdossy, 2006; Favre et al., 2004; Salvadori and De Michele, 2010; Madadgar and Moradkhani, 2013, and 2014) brought the technique to study hydrological aspects such as drought, groundwater monitoring etc., amongst others. 
Favre et al. (2004) modeled the joint distribution between flows and volumes through copula functions to study the flood frequency estimation. They modelled the flows and volumes considering different marginal distribution for two variables. Bárdossy (2006) implemented copulas to describe spatial variability of groundwater quality parameters. Salvadori and De Michele (2010) introduced multivariate extreme value models, based on the copula functions, and assessed a new definition for the multivariate return period. (Madadgar et al., 2014) applied copula functions for post-processing of streamflow simulations and has detailed capabilities of copula functions to describe joint distribution of observation and simulation. Recently, (Schefzik et al., 2013) implemented copula functions to address the relation between raw ensemble climate forecasts and postprocessed forecasts using Bayesian model averaging. (Madadgar and Moradkhani, 2014) brought conditional probability based on copula functions to Bayesian model averaging territory and introduced Cop-BMA procedure.

Madadgar et al. (2014) proposed that copulas have the potential application in postprocessing hydrological simulation based on observation and modeled streamflow, since copulas are able to construct the joint distribution between two variables with any level of correlation and independency,. The study demonstrated that copula could deliver more accurate and skillful simulation as compared to the common post-processing techniques, such as quantile mapping. It would thus be advantageous to adapt EPP with potential application of Copula functions. In the present study, we have evaluated the capability of Copula functions to describe the joint distribution of observation and climatological forecast. The main assumptions, in pre- processing the forecasts based on the historical 
observation, includes correlation of observation and forecast and thus assumption of same future behavior of the system as historical. Therefore, Copulas are relaxing these two fundamental assumptions and be able to build ensemble forecasts based on historical observations and climatological forecasts.

The following study is organized in 8 chapters, Introduction is followed by methodology, wherein the two techniques namely Meta-Gaussian and Copula are detailed. Third chapter focus on forecast verification methods followed by study area and dataset description in fourth and fifth chapters, respectively. Results of study are presented in chapter 6 with discussion and concluding remarks in chapter 7. Lastly, the ideas for future work are outlined in chapter 8 . 


\section{Methodology}

In the present study, we have developed and evaluated two techniques to assess their strength for EPP i.e. post-processing of climatological parameters. First technique termed as Mixed Bivariate meta-Gaussian distribution (MBG), introduced by (Wu et al., 2011) is a powerful and simple procedure for EPP. We used said procedure as the benchmark, to appraise the robustness and reliability of the new technique we developed based on copula functions. This provides users with flexibility of fitting any marginal

distribution to the observation and forecast. Copula functions are able to build the joint distribution between two variables with any level of dependency. Before setting up the techniques, it would be beneficial to give a brief overview over the mathematical theories behind copula functions whereas readers are suggested to refer to (Wu et al., 2011) for details on MBG.

\subsection{Copulas}

\subsubsection{Mathematical Background}

Multi-dimensional distribution studies require functions to be defined on the unit cube, which is capable of connecting such distributions to their one-dimensional margins. (Sklar, 1959) introduced copula as the function that links a multidimensional distribution to its one-dimensional margins. Mathematically, copula is defined as the joint Cumulative Density Function (CDF) of two univariate marginal distribution function by (Joe, 1997; Nelsen, 1999) . The multivariate copula $\mathrm{C}$ is defined as:

$$
C:[0,1]^{n} \rightarrow[0,1]
$$


Wherein following conditions should be satisfied by copula functions:

$$
\begin{aligned}
& \qquad:[0,1]^{n} \rightarrow[0,1] \\
& C\left(u_{1}, u_{2}\right)+C\left(v_{1}, v_{2}\right)-C\left(u_{1}, v_{2}\right)-C\left(v_{1}, u_{2}\right) \geq 0 \text { if } u_{1} \geq v_{1} \text { and } u_{2} \geq v_{2} \\
& \text { with } u \text { and } v \text { as marginals of random variables. }
\end{aligned}
$$

In n-dimension space, we have:

$$
\begin{aligned}
& F\left(x_{1}, x_{2}, \ldots, x_{n}\right)=C\left[F_{X_{1}}\left(x_{1}\right), F_{X_{2}}\left(x_{2}\right), \ldots, F_{X_{n}}\left(x_{n}\right)\right]=C\left(u_{1}, u_{2}, \ldots, u_{n}\right) \\
& C\left(u_{1}, u_{2}, \ldots, u_{n}\right)=\operatorname{Pr}\left\{U_{1} \leq u_{1}, \ldots, U_{2} \leq u_{2}\right\}
\end{aligned}
$$

Based on the derivative of cdf of copula, the pdf (probability distribution function) of copula is defined as below:

$$
c\left(u_{1}, \ldots, u_{n}\right)=\frac{\boldsymbol{\partial}^{n} C\left(u_{1}, \ldots, u_{n}\right)}{\partial u_{1} \ldots \partial u_{1}}
$$

Furthermore, the joint density function can be also broken down as follows:

$$
f\left(x_{1}, \ldots, x_{n}\right)=c\left(u_{1}, \ldots, u_{n}\right) \prod_{i=1}^{n} f_{X_{i}}\left(x_{i}\right)
$$

\subsubsection{Types of Copulas}

Copula functions are categorized into several categories (Nelsen, 1999). Two of the families that are applicable in this study are Archimedean and Elliptical.

Elliptical copulas are capable of capturing the pair-wise correlations among the variables with any level of correlation; however, this family does not have a closed form expression. On the other hand, the Archimedean copulas provide the closed form 
expression but cannot preserve all the pair-wise correlations. While modeling with Elliptical copulas, usage of the covariance matrix is suggested they are able to model the variables with positive-defined correlation matrix. Archimedean copulas are classified into two categories, symmetric and asymmetric functions. Table 1 provides a summary of the formulation and differences between different copula functions. For more detailed description on copula functions, readers are referred to (Nelsen, 1999; Sklar, 1959) .

Table 1 .Copula functions description used in this study

\begin{tabular}{|c|c|c|c|}
\hline Name & Bivariate Copula $C(u, v)$ & $\begin{array}{l}\text { Param } \\
\text { eter }\end{array}$ & $\begin{array}{l}\text { Copula } \\
\text { Family }\end{array}$ \\
\hline Gaussian & $\begin{array}{l}\int_{-\infty}^{\emptyset^{-1}(u)} \int_{-\infty}^{\emptyset^{-1}(v)} \frac{1}{2 \pi\left(1-\rho^{2}\right)^{\frac{1}{2}}} \exp \left\{1+\frac{x^{2}+y^{2}-2 \rho x y}{v\left(1-\rho^{2}\right)}\right\} d x_{1} d x_{1} \\
\rho: \text { Linear correlation coefficient } \\
\varnothing: \text { Standard normal cumulative distribution function }\end{array}$ & $\begin{array}{l}x_{1}, x_{2} \\
\in R\end{array}$ & $\begin{array}{c}\text { Elliptica } \\
1\end{array}$ \\
\hline $\mathrm{t}$ & $\begin{array}{l}\int_{-\infty}^{\emptyset^{-1}(u)} \int_{-\infty}^{\emptyset^{-1}(v)} \frac{1}{2 \pi\left(1-\rho^{2}\right)^{\frac{1}{2}}} \exp \left\{1+\frac{x^{2}+y^{2}-2 \rho x y}{v\left(1-\rho^{2}\right)}\right\}^{-\frac{v+2}{2}} d x_{1} d x_{1} \\
\rho: \text { Linear correlation coefficient }\end{array}$ & $\begin{array}{l}x_{1}, x_{2} \\
\in R\end{array}$ & $\begin{array}{c}\text { Elliptica } \\
1\end{array}$ \\
\hline Frank & $-\frac{1}{\theta} \ln \left[1+\frac{\left(e^{-\theta_{u}}-1\right)\left(e^{-\theta_{v}}-1\right)}{\exp (-\theta)-1}\right]$ & $\begin{array}{l}\theta \\
\in R \\
\backslash\{0\}\end{array}$ & $\begin{array}{l}\text { Archim } \\
\text { edean }\end{array}$ \\
\hline
\end{tabular}

\subsubsection{Choice of copula functions for dataset}

Goodness of Fit (GoF) test is usually employed to access the suitability of particular copula to the dataset. There are several other techniques for testing applicability of copula 
function. The simplest one includes visual inspection of the applied copula function and its comparison to empirical one. In that case, scatterplot would follow the line 1:1 if the applied copula could describe the relationship between the variables exquisitely. However, it is time consuming and not trustworthy. Therefore, (Genest et al., 2009) used the GoF test statistics based on the distance between empirical and parametric copulas. Cramer-von Mises and Komogorov-Smimov statistics (Anderson, 1962) were used as the measure the distance between theoretical and empirical copulas. Mathematically, the Cramer-von Mises statistic is described as below:

$$
\begin{aligned}
& S_{n}=\int_{u} \Delta C_{n}(n)^{2} d C_{n}(u) \\
& \Delta C_{n}=\sqrt{n}\left(C_{n}-C_{\theta_{n}}\right)
\end{aligned}
$$

Where $C_{n}$ is the empirical copula with sample size of $n$, and $C_{\theta n}$ is the theoretical copula estimated for a sample size of $\mathrm{n}$. The null hypothesis assumes that the theoretical copula fits the data. Therefore, copula with the greatest $\mathrm{p}$-value or the smallest $\mathrm{S}_{\mathrm{n}}$ is desirable.

\subsection{Methodology used and flow-chart}

In the present study we have evaluated two EPP techniques for reliability of forecast and reduction of uncertainty. The methodology consists of four main steps (figure 1):

(1) Data classification and Marginal distribution application

(2) Constructing the ensemble members: 
(a) Based on the MBG

(b) Based on the copula functions

(3) Reconstruction of the ensemble members

(4) Validation

Each of the above steps are detailed in next sections of the chapter.

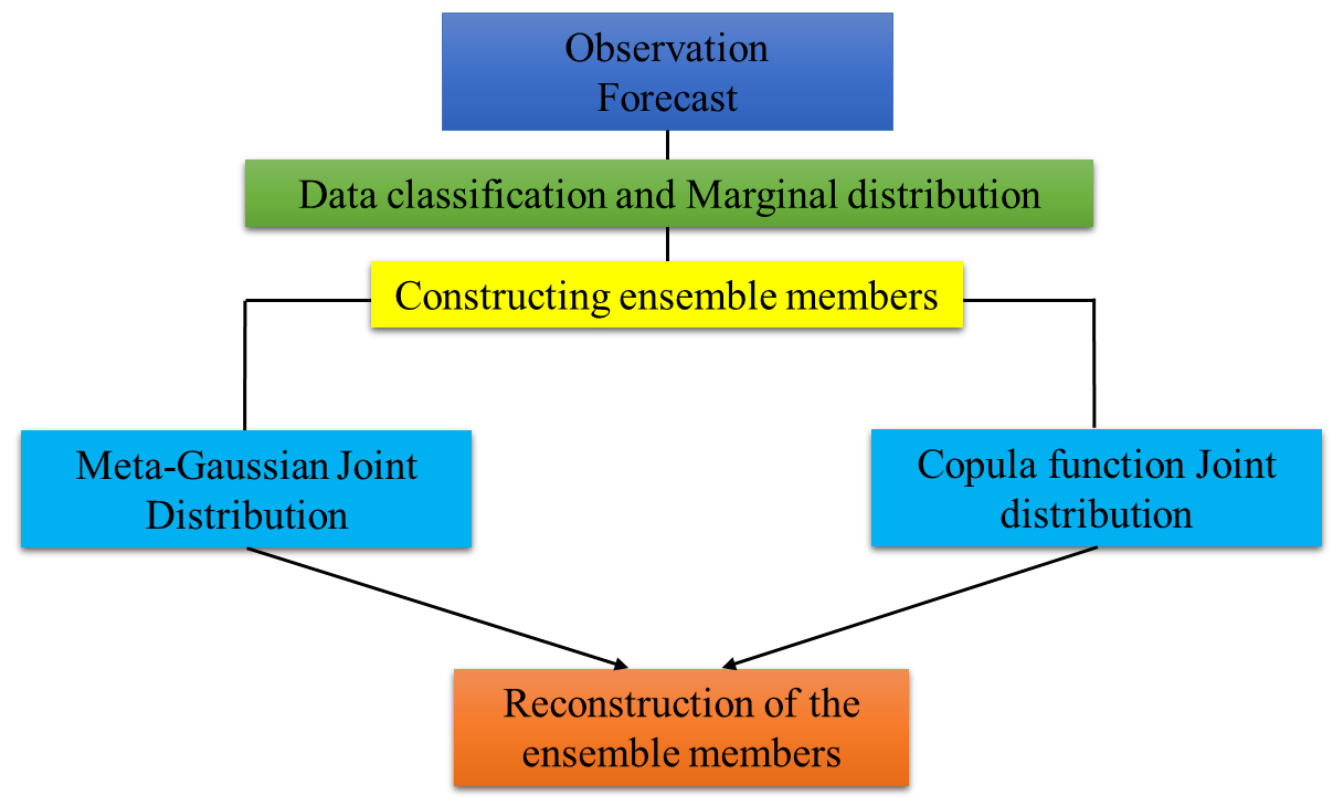

Figure1.Constructing the transfer function in the calibration period.

\subsection{Data classification and Marginal distribution}

The data is classified into 12 monthly categories in the calibration period and same procedure is applied to each of the monthly series. The procedure starts with applying/fitting pertinent marginal distribution to both observation and forecast. Climatological variable is fitted to four distributions, including Exponential, Lognormal, Weibull, and Gamma. The motivation for this selection is to have distributions which are 
covering positive values of precipitation and similarity to sampling technique (from cdf) in meta-Gaussian procedure. To estimate the parameters of the marginal distributions, Maximum Likelihood Estimation method is employed. Kolmogorov-Smirnov (K-S) and Akaike Information Criterion (AIC) tests are utilized to determine best marginal distribution. AIC test is used for comparison of quality of a statistical model to describe the variables (Aho et al., 2014).

$$
A I C=-2 \max (\ln L)+2 K
$$

Where $\mathrm{K}$ is the number of model parameters, and $\mathrm{L}$ is the maximized value of the likelihood function. The model with the smallest AIC value has the potential to be the best marginal distribution. Since the AIC test does not provide with a null hypothesis it alone can't be used to choose best distribution. Hence a combination of AIC and K-S is used to select the marginal distribution.

$\mathrm{K}-\mathrm{S}$ test is defined as a non-parametric test to measure the distance between the empirical and calculated CDF (Stephens, 1974).

$$
M=\operatorname{Max}\{|E(x)-R(x)|\}
$$

Where $\mathrm{E}(\mathrm{x})$ and $\mathrm{R}(\mathrm{x})$ are the empirical and calculated CDFs. The null hypothesis for this test assumes that the variable is a part of the calculated distribution. Details of the procedure used in this section are outlined in Figure 2. 


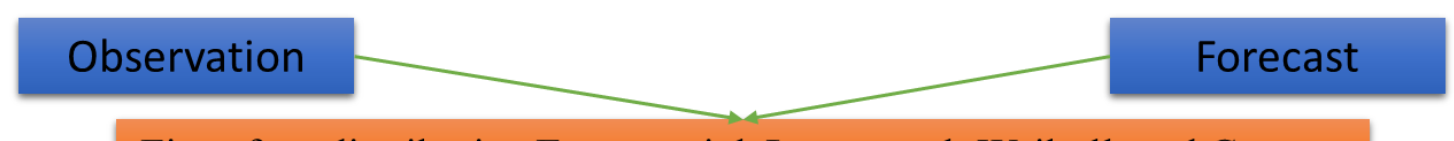

Fit to four distribution Exponential, Lognormal, Weibull, and Gamma

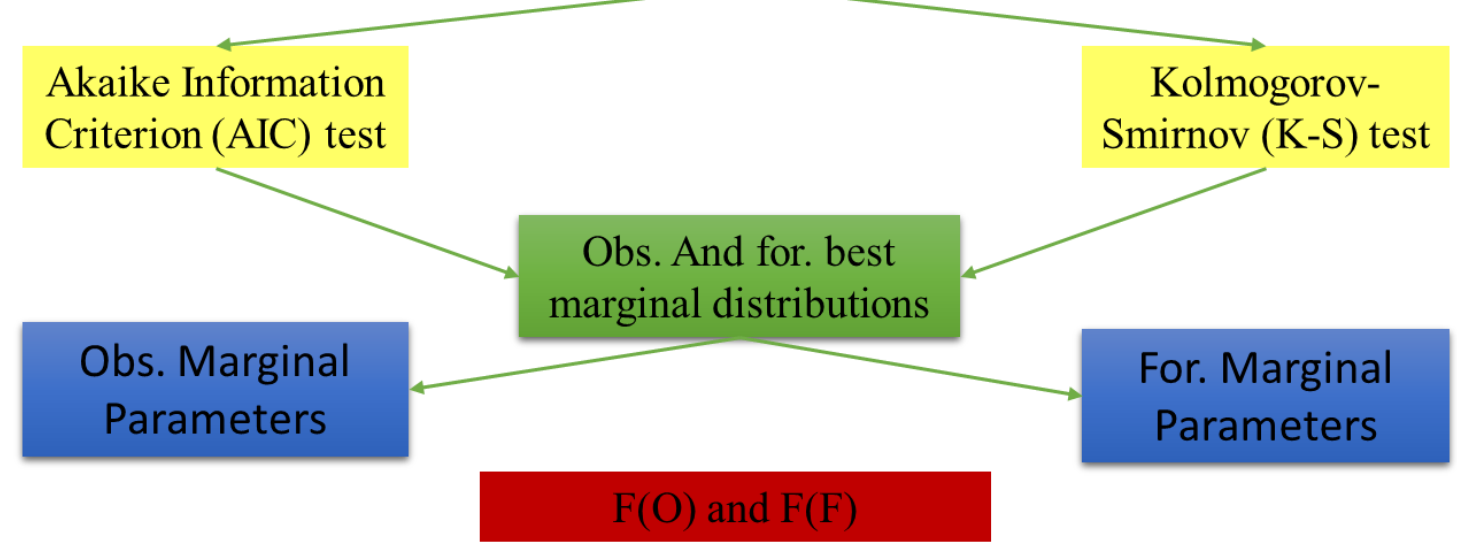

Figure 2. Find the best marginal distributions that describe the observation and forecast, separately.

\subsection{Ensemble construction}

This step includes construction of conditional probability distribution and sampling from to generate the desirable ensemble members, as described in figure 3. 


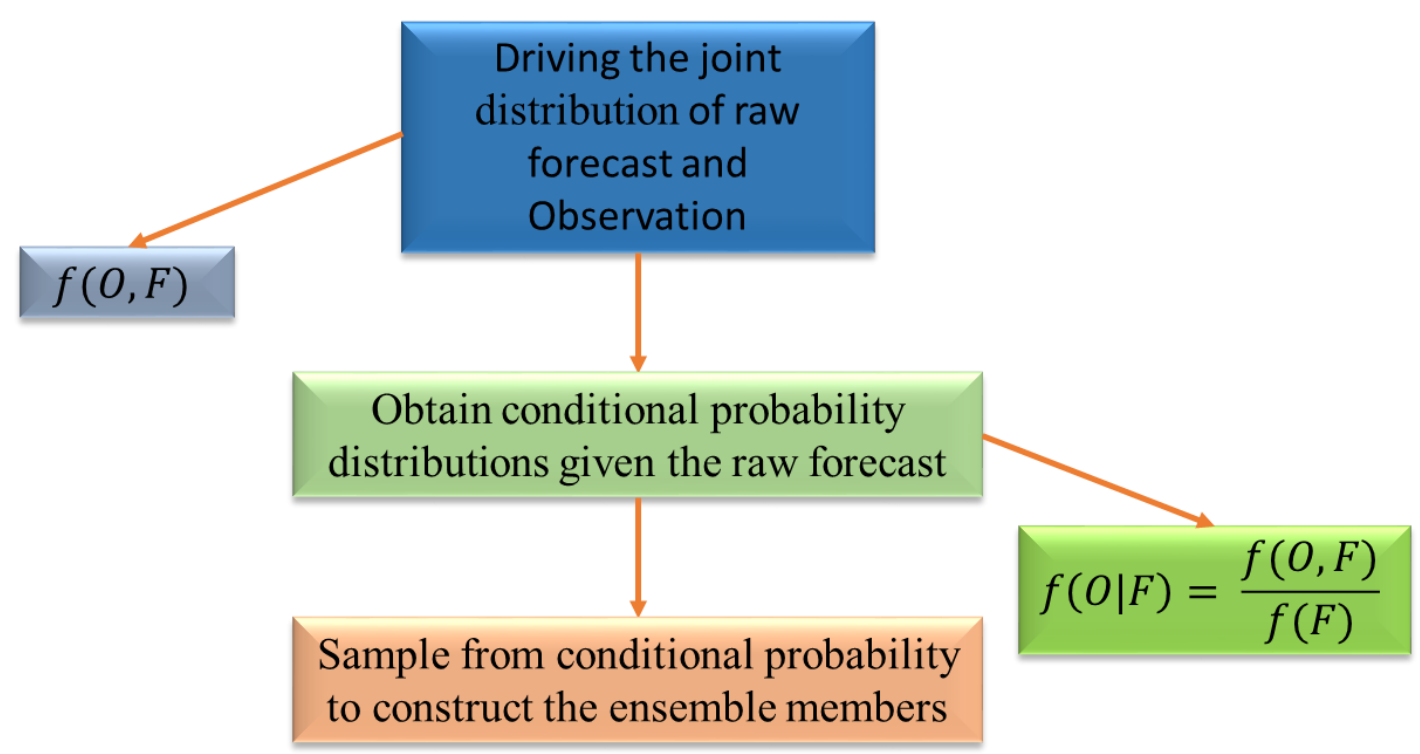

Figure 3.Ensemble Construction overview

2.4.1. The Bivariate meta-Gaussian Distribution Model (MBG)

A joint $\mathrm{CDF}$ of two variables $\mathrm{O}$ and $\mathrm{F}$ (observation and raw forecast) is constructed and CDF is sampled to generate $\mathrm{n}$ number of ensemble members at each time step.

$$
F(f, O)=P(F \leq f, O \leq o)
$$

$F(f)$ and $F(O)$ presents the CDF of the raw forecast and observation. Based on the assumption of normal distribution for two variables, Normal Quantile Transform (NQT) is used to draw the marginal distributions to normal space.

$$
\begin{array}{r}
Z=Q^{-1}(F(F)) \\
W=Q^{-1}(F(O))
\end{array}
$$


Where $\mathrm{Z}$ and $\mathrm{W}$ will replace the $\mathrm{F}(\mathrm{F})$ and $\mathrm{F}(\mathrm{O})$ in the calculations. The joint distribution of the $\mathrm{Z}$ and $\mathrm{W}$ is not bivariate essentially. If $\mathrm{B}$ is considered as bivariate standard normal distribution function:

$$
H(x . y ; \rho) \equiv B(z, w ; \rho)
$$

Where $\rho$ states the Pearson product-moment correlation coefficient between $\mathrm{Z}$ and W. assuming the $(\mathrm{Z}, \mathrm{W})$ is standard normal, we have:

$$
P(Z \leq z, W \leq w)=B(z, w ; \rho)
$$

$\mathrm{H}(\mathrm{x}, \mathrm{y} ; \rho)$ is bivariate meta-Gaussian distribution of $\mathrm{X}$ and $\mathrm{Y}$, introduced by (Kelly and Krzysztofowicz, 1997). We can assume that:

$$
F(x, y) \approx H(x, y ; \rho)
$$

In the conditional distribution of $\mathrm{Y}$ given $\mathrm{X}=\mathrm{x}$ aspect, it is given:

$$
H_{Y \mid X}(y \mid x)=Q\left(\frac{w-\rho z}{\left(1-\rho^{2}\right)^{\frac{1}{2}}}\right)
$$

For any probability $\mathrm{p}=H_{Y \mid X}\left(y_{P \mid X} \mid x\right)$, the p-probability conditional quantile of $\mathrm{Y}$ given $\mathrm{X}=\mathrm{x}$, we have:

$$
Y_{p \mid x}=F_{Y}^{-1}\left(Q\left(\rho z+\left(1-\rho^{2}\right)^{\frac{1}{2}} Q^{-1}(p)\right)\right)
$$

In the present study, $\mathrm{Y}$ is taken as the observation in the hindcast period and $\mathrm{X}$ in raw forecast. The equations are solved in the calibration period, followed by estimation of stratified samples of observation given forecast using ensemble member. 


\subsubsection{Copula Ensemble Construction}

In this study, the conditional joint distribution based on the copula functions is being employed to generate ensemble precipitation forecast. As per the mathematical theory of copula functions, we have:

$$
f\left(o_{t} \mid s_{t}\right)=\frac{c\left(U_{O}=u_{o}, U_{S}=u_{s}\right) f\left(s_{t}\right) f\left(o_{t}\right)}{f\left(s_{t}\right)}=c\left(U_{O}=u_{o}, U_{S}=u_{s}\right) f\left(o_{t}\right)
$$

Where; $f\left(o_{t} \mid s_{t}\right)$ is the joint PDF of the observation and simulation at time t, $f\left(s_{t}\right)$

and $f\left(o_{t}\right)$ are the marginal PDF of the simulation and observation. $c(.,$.$) is representing$ the copula pdf of the marginal distributions.

In EPP, we estimate the type of the copula function to be used based on the observation and forecast, in the calibration period. To build conditional joint distribution, (Madadgar and Moradkhani, 2014) suggested Monte Carlo sampling from copula density function, where $u_{s}$ is fixed at time step $t$ and $u_{o}$ is changing in sample space. The same is built related to observation in calibration period. In principle, for both pre-processing and post-processing, the variable behavior is assumed to be the same as was in past. Therefore, $c\left(U_{O}=u_{o}, U_{S}=u_{s}\right)$ is being built to achieve the left side of the equation (18).

Once we have the conditional joint PDF of the sample data given the forecast, it will lead us to sample the ensemble member of the PDF. Inverse CDF method is applied to capture joint CDF from PDF. A uniform sample of $\mathrm{n}$ probability, between zero and one is generated, followed by interpolation of probability samples between the joint CDF and 
sampled variable, the $\mathrm{n}$ number of ensemble members at the time step $\mathrm{t}$ is created. Details of copula are outlined in flowchart (Figure 4).

\subsection{Ensemble reordering}

Schaake Shuffle was proposed by(Clark et al., 2004) to preserve the precipitation variability in time and space wherein the ensemble members are reordered in relation to the historical observation. In the present study, to preserve the space variability, we have used gridded data for both the observation and forecast with the same grid size. For time variability, we applied Schaake Shuffle to both generated ensemble precipitation forecasts.

Mathematically, Let $\mathrm{X}$ be the ensemble forecast at time $\mathrm{t}$ and $\mathrm{Y}$ be the historical observation, $\mathrm{x}$ and $\mathrm{y}$ are denoted as the sorted ensemble forecast and sorted observation:

$$
\begin{aligned}
& X=\left\{x_{1}, x_{2}, \ldots, x_{n}\right\} \\
& x=\left\{x_{(1)}, x_{(2)}, \ldots, x_{(n)}\right\} \quad x_{(1)}<x_{(2)}<\cdots<x_{(n)} \\
& Y=\left\{y_{1}, y_{2}, \ldots, y_{n}\right\} \\
& y=\left\{y_{(1)}, y_{(2)}, \ldots, y_{(n)}\right\} \quad y_{(1)}<y_{(2)}<\cdots<y_{(n)}
\end{aligned}
$$

If $\mathrm{B}$ is consider as a vector with the indices corresponding to the observation vector, $\mathrm{Y}$, in $\mathrm{y}$.

$$
\begin{aligned}
& r=1, \ldots, n \\
& q=B[r] \\
& X^{P P}=\left(x_{1}^{P P}, x_{2}^{P P}, \ldots, x_{n}^{P P}\right)
\end{aligned}
$$




$$
\text { where } x_{q}^{p p}=x_{(r)}
$$

$\mathrm{X}^{\mathrm{PP}}$ is the organized ensemble precipitation forecast.

2.6. Validating the procedure

Finally, in validation period, the same procedure is applied to the forecast data with an assumption of lack of observation. The parameters and functions that were estimated in the calibration period are transferred to the forecast in the validation period. Thus providing with an opportunity to investigate the applicability of said techniques for future precipitation forecast. 


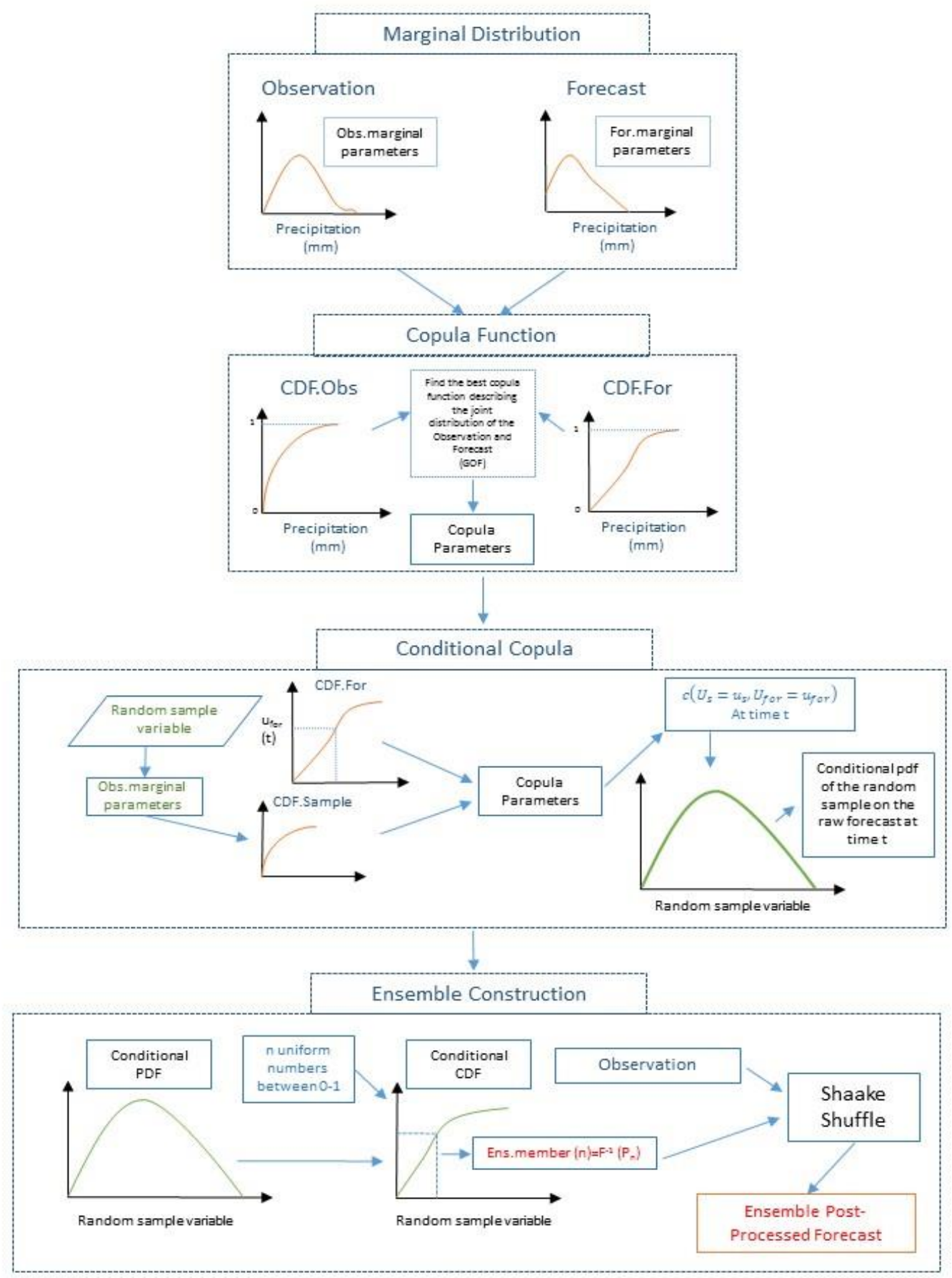

Figure 4 .Copula based EPP scheme. The procedure starts with fitting marginal distribution to observation and forecast in the historical period. In the second step, the best copula function, which describes the joint distribution between the observation and forecast, is being chosen through GoF test. Next step would be building the conditional pdf by sampling from copula pdf, with fixed forecast at time $t$ and moving in the sample vector created based on historical observation. Lastly, the ensemble precipitation is constructed by sampling from the conditional pdf and reconstructing it by Schaake Shuffle. 


\section{Forecast Verification}

Verification is a process of assessing the quality of a forecast in comparison to the existing observation. In order to do so, verification measure have been introduced and developed through time (DeChant and Moradkhani, 2014). These measures usually fall into two categories namely deterministic and probabilistic. Deterministic measures demonstrate the errors in forecast procedure; however, they are prone to bias for overconfident and under-confident forecasts. On the other hand, probabilistic measures focus

on the usefulness of generated forecast and thus give more information about the reliability of forecast (DeChant and Moradkhani, 2015). In this study, owing to usefulness of the methods, the results are evaluated with both deterministic and probabilistic frameworks.

\subsection{Deterministic Measures}

\subsubsection{Mean Absolute error (MAE)}

MAE is a quantity for measure of closeness of the forecast to the observation. It is calculated in the dimension of the variables.

$$
M A E=\frac{1}{N} \sum_{i=1}^{n}\left|Y_{i}^{s i m}-Y_{i}^{o b s}\right|
$$

MAE ranges between zero and $\infty$ with optimal value zero. Since this measure is not a normalized, it would be hard to compare the results and make a conclusion about the performance of the forecast over observation.

\subsubsection{Root Mean Square Error (RMSE)}


RMSE is a square root of the mean of square of all of the errors. Therefore, it is amplifying the large errors in forecast. However, like MAE, it is not dimensionless so it would be hard to interpret the results.

$$
R M S E=\sqrt{\frac{1}{N} \sum_{i=1}^{n}\left(Y_{i}^{s i m}-Y_{i}^{o b s}\right)^{2}}
$$

\subsubsection{Nash-Sutcliffe Efficiency (NSE)}

NSE is normalized measure that assesses the predictive power of forecast versus observation (Nash and Sutcliffe, 1970). To some extent, it is evaluating the residual variance in comparison with observed variance. NSE compares the plot of observed and simulated data to 1:1 line. It is computed as below:

$$
N S E=1-\left[\frac{\sum_{i=1}^{n}\left(Y_{i}^{o b s}-Y_{i}^{s i m}\right)^{2}}{\sum_{i=1}^{n}\left(Y_{i}^{o b s}-\overline{Y^{o b s}}\right)^{2}}\right]
$$

In this study $Y_{i}^{s i m}$ is taken as the mean ensemble at time t. $Y_{i}^{\text {obs }}$ is the observation at the same time and $\overline{Y^{o b s}}$ is the mean of the observation for the period of study. NSE ranges from $-\infty$ to one. Values between zero and one are acceptable with one being the optimal value of measure. This measure is used to evaluate the overall performance of the ensemble forecast versus the raw forecast.

\subsubsection{Kling-Gupta Efficiency (KGE)}

KGE was proposed by (Gupta et al., 2009) as a modified version of NSE, which is capable of capturing the correlation, bias, and variability at the same time. 


$$
\begin{gathered}
K G E=1-E D \\
E D=\sqrt{(r-1)^{2}-(\alpha-1)^{2}-(\beta-1)^{2}}
\end{gathered}
$$

ED is defined as the Euclidean distance between two variables, $r$ represents the ratio of the coefficient of variance of the forecast to the observation, $\alpha$ is correlation between forecast and observation, and $\beta$ is the ratio between the mean of forecast and observation.

\subsection{Probabilistic Measures}

\subsubsection{Continues Ranked Probability Skill Score (CRPSS)}

(Hersbach, 2000) introduced Continues Ranked Probability Score (CRPS) as the integral of the Brier Score over all possible thresholds. However, the measure is not dimensionless, therefore, CRPSS has introduced as the normalized version of CRPS.

$$
\text { CRPS }=\frac{1}{n} \sum_{i}^{n} \int_{x=-\infty}^{x=\infty}\left(F_{i}^{s i m}(x)-F_{i}^{o b s}(x)\right)^{2} d x
$$

Where $F_{i}^{\text {sim }}(x)$ is the forecast probability i.e. cdf for the forecast at time step $\mathrm{i}$ and $F_{i}^{o b s}(x)$ is the observation's cdf. In the deterministic case of study, CRPS is transformed to MAE. The optimal value for CRPS is zero.

$$
C R P S S=\frac{\overline{C R P S_{\text {ref }}}-\overline{C R P S_{\text {forecast }}}}{\overline{C R P S_{\text {ref }}}}
$$

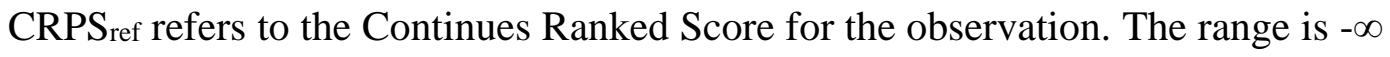
to one with optimal value as one.

\subsubsection{Normalized Root Mean Square Error (NRR)}


According to (Anderson, 2001), NRR is a score to measure the ensemble dispersion. Therefore, it indicates confidence of the ensemble mean to be a representative of the ensemble forecast.

$$
\begin{aligned}
& R_{1}=\sqrt{\frac{1}{T} \sum_{t=1}^{T}\left[\left(\frac{1}{n} \sum_{i=1}^{n} y_{i}^{s i m}\right)-O_{t}\right]^{2}} \\
& R_{2}=\frac{1}{n} \sum_{i=1}^{n} \sqrt{\frac{1}{T} \sum_{t=1}^{T}\left[y_{t}^{i}-O_{t}\right]^{2}} \\
& R a=\frac{R_{1}}{R_{2}} \quad E\left[R_{a}\right]=\sqrt{\frac{(n+1)}{2 n}} \\
& N R R=\frac{R a}{E\left[R_{a}\right]}
\end{aligned}
$$

Where, $\mathrm{n}$ and $\mathrm{T}$ are the ensemble size and period of study, $\mathrm{R}_{1}$ is the time-averaged RMSE of the ensemble mean, $\mathrm{R}_{2}$ is the average RMSE of the ensemble members, and $E\left[R_{a}\right]$ is the normalizing factor. The optimal value of NRR is one. In other cases, NRR>1 represents the ensemble with too little spread and $\mathrm{NRR}<1$ indicates that the ensemble has too much spread. The method have used earlier to evaluate quality of ensemble streamflow by (Moradkhani et al., 2005a; Moradkhani et al., 2005b).

\subsubsection{Exceedance Ratio}

Exceedance Ratio is a factor that investigates the spread (wideness) of the prediction quantiles, introduced by (Borga, 2002). The uncertainty bounds in the ensemble 
can specify predictive capability and thus precision of the ensemble. To some extent, ensemble with wide spread has high predictive ability despite low precision. On the other hand, the one with narrow spread risk the ensemble with high amount of bias (Moradkhani et al., 2006).

$$
E R_{n}=\frac{N_{\text {exceedance }}^{n}}{T} * 100
$$

$\mathrm{ER}_{\mathrm{n}}$ is the exceedance ratio for the $\mathrm{nth}$ quantile, $N_{\text {exceedance }}^{n}$ is number of times during the study period, $\mathrm{T}$, the forecast falls out of the nth percentile bound.

(DeChant and Moradkhani, 2014) brought in an extension to the exceedance ratio which is adapted to examine the ensemble products. It evaluates the measuring capabilities of cumulative probability of each observation, given its forecast at time t. Consequently, the Exceedance ratio becomes the probability with which the observation falls out of the interquartile, which in this study would be $95 \%$ percentile. 


\section{Study Area}

The procedures in EPP introduced above are evaluated in three basins of Western United States, figure 5. Upper Colorado River Basin is largest amongst three and is located downstream of the Lake Powell. It covers portions of Wyoming, Utah, Arizona and New Mexico. The drainage area of the basin is about $280,000 \mathrm{~km}^{2}$. The basin is known to have semi-arid climatology, where the precipitation occurs in the upper elevation as snow with average annual amount of $164 \mathrm{~mm}$.

The Oxbow River Basin is located in the southeast part of the Columbia River Basin (CRB). It is known as one of the biggest sub-basins of CRB. It is located in the semi-arid portion of CRB, receiving $493 \mathrm{~mm}$ averaged annual precipitation. The basin drains an area of approximately $180,000 \mathrm{~km}^{2}$.

Lastly, the Rouge River Basin is located in southwestern Oregon with the drainage area of $5,318 \mathrm{~km}^{2}$. It drains between the Cascade Mountains and the Pacific Ocean and is known as a costal basin with relatively high average annual precipitation of $970 \mathrm{~mm}$. Since this basin is located near the ocean, the observation for the western-part is not available throughout the study area. 


\section{Study Basins}

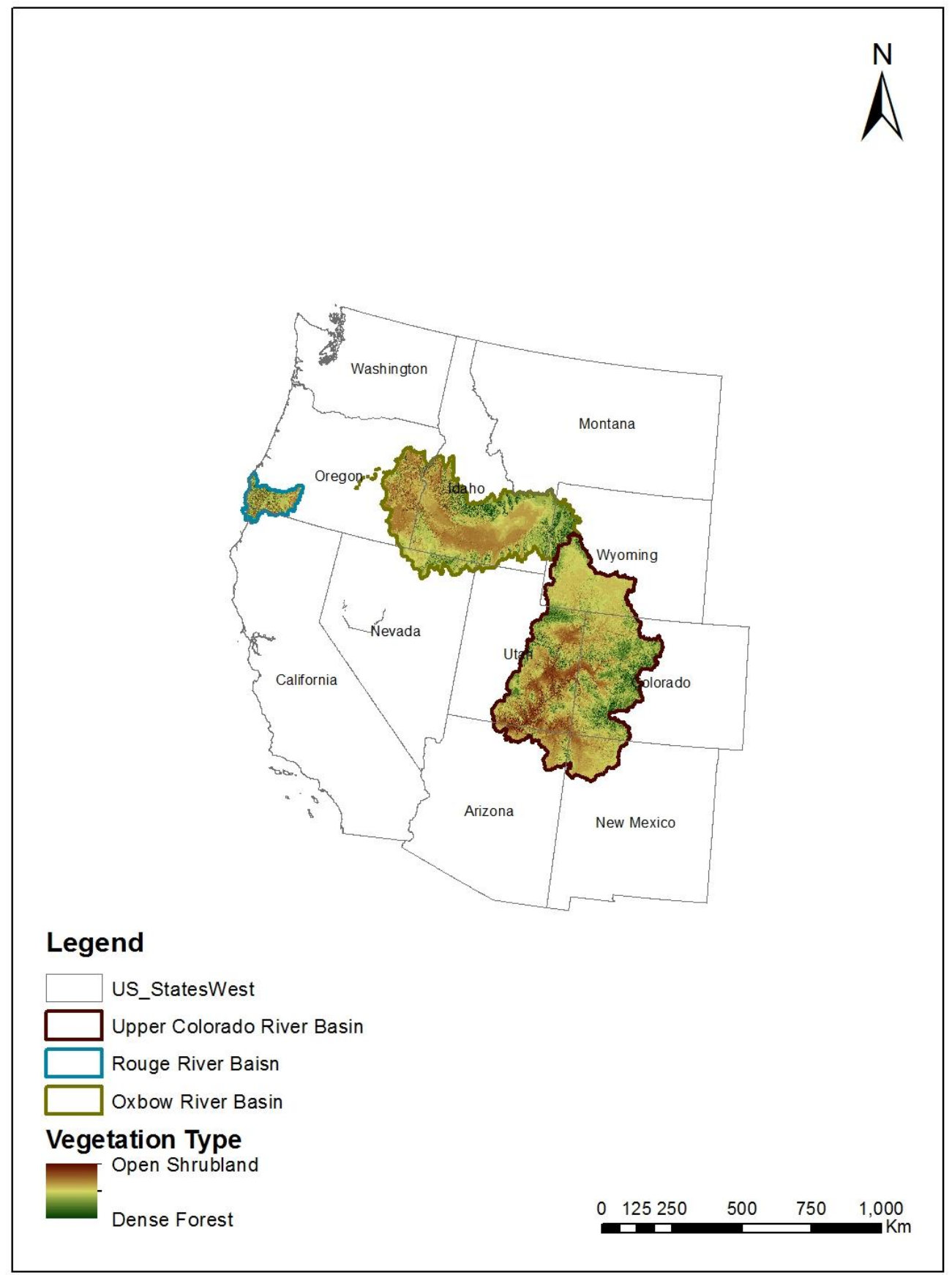

Figure 5 .Map of western USA with the 3 study basins (Rouge River Basin, Oxbow River basin, and Upper Colorado River Basin) including type of vegatation in the basins. 


\section{Data}

The present study is data intensive. The observation and forecast have been divided into two period, calibration and validation period, respectively. The observation dataset is chosen from the PRISM Climate Group, generated at Oregon State University and available at http://prism.oregonstate.edu. PRISM observations are result of gathering climate observations from a wide range of monitoring networks, and developed as special climate datasets for short and long-term climate patterns over the United States. In this study, monthly precipitation was downloaded for the period of 1979-2009, including rain and melted snow. This data is available over $4 \mathrm{~km}$ grid cells.

The National Centers for Environmental Prediction (NCEP) product called Climate Forecast System Reanalysis (CFSR) was used to gather the meteorological forecast for the study. CFSR is available as a global, high-resolution, coupled atmospheric-ocean-land surface-sea ice system over the period of 1979 to 2009 (Saha et al., 2010). CFSR is now under the extension for the real time product into future, in the content of CFSv2. For this study, the precipitation rate over the $0.5^{\circ} \times 0.5^{\circ}$ for the monthly mean of forecast of 6-hour averaged was utilized.To prepare the observation and forecast for the study, the observation data is re-gridded to $0.5^{\circ} \times 0.5^{\circ}$ resolution. The forecast data is converted into precipitation amount (in $\mathrm{mm}$ ) for pre-processing. Table (2) shows summary of data.

Table 2 . Summary of the monthly precipitation data used in the study.

\begin{tabular}{|c|c|c|c|c|}
\hline Climate Variable & Data Set & Source & Calibration Period & Validation Period \\
\hline $\begin{array}{c}\text { Monthly } \\
\text { Precipitation } \\
(\mathrm{mm})\end{array}$ & Observation & PRMS & $1979-1999$ & $2000-2009$ \\
\cline { 2 - 5 } & Forecast & CFSR & $1979-1999$ & $2000-2009$ \\
\hline
\end{tabular}




\section{Designed Experiment and Result}

\subsection{Raw Forecast}

Before evaluation of EPP procedures it is advised to study the performance of raw forecast in comparison to observation. Herein raw forecast predictions are assessed through the deterministic forecast verification framework, namely MAE, RMSE, KGE, and NSE. Results are depicted in figures (6) to (9) respectively for the deterministic measure used.

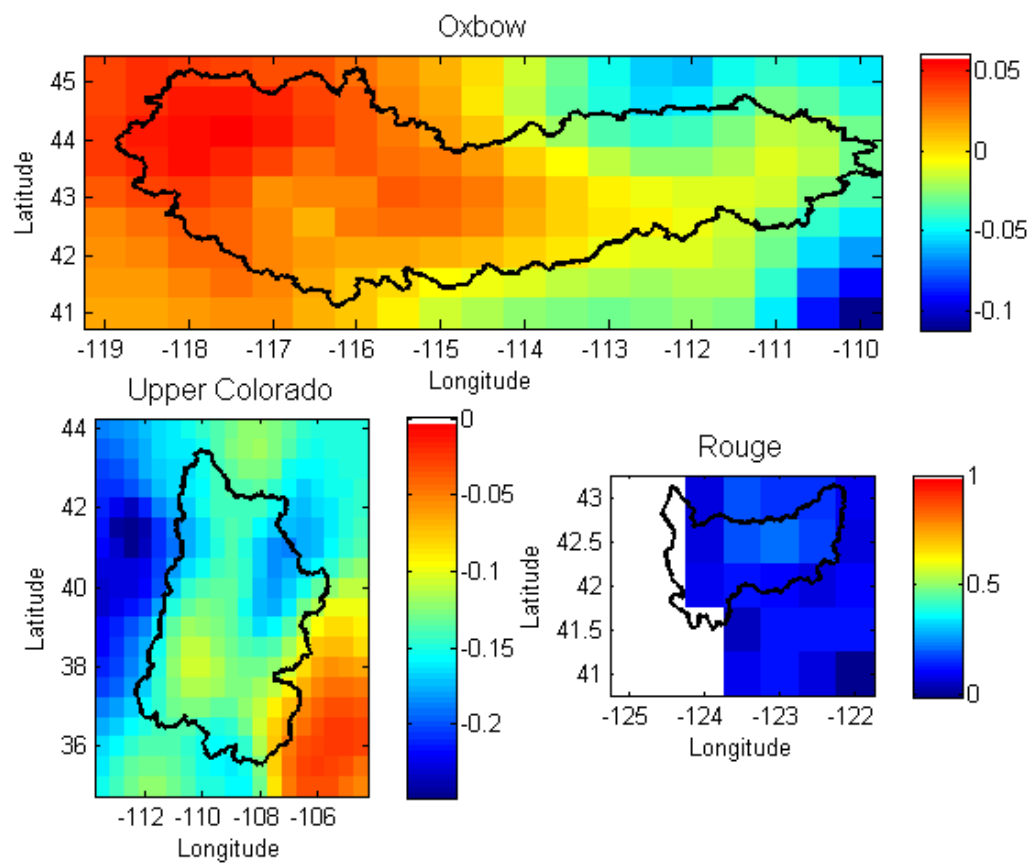

Figure 6 . NSE measure calculated between the observation and raw forecast for monthly precipitation over the calibration period (1979-1999) for the study basin, the white grid cells in Rouge River basin are the ones with missing observation. 

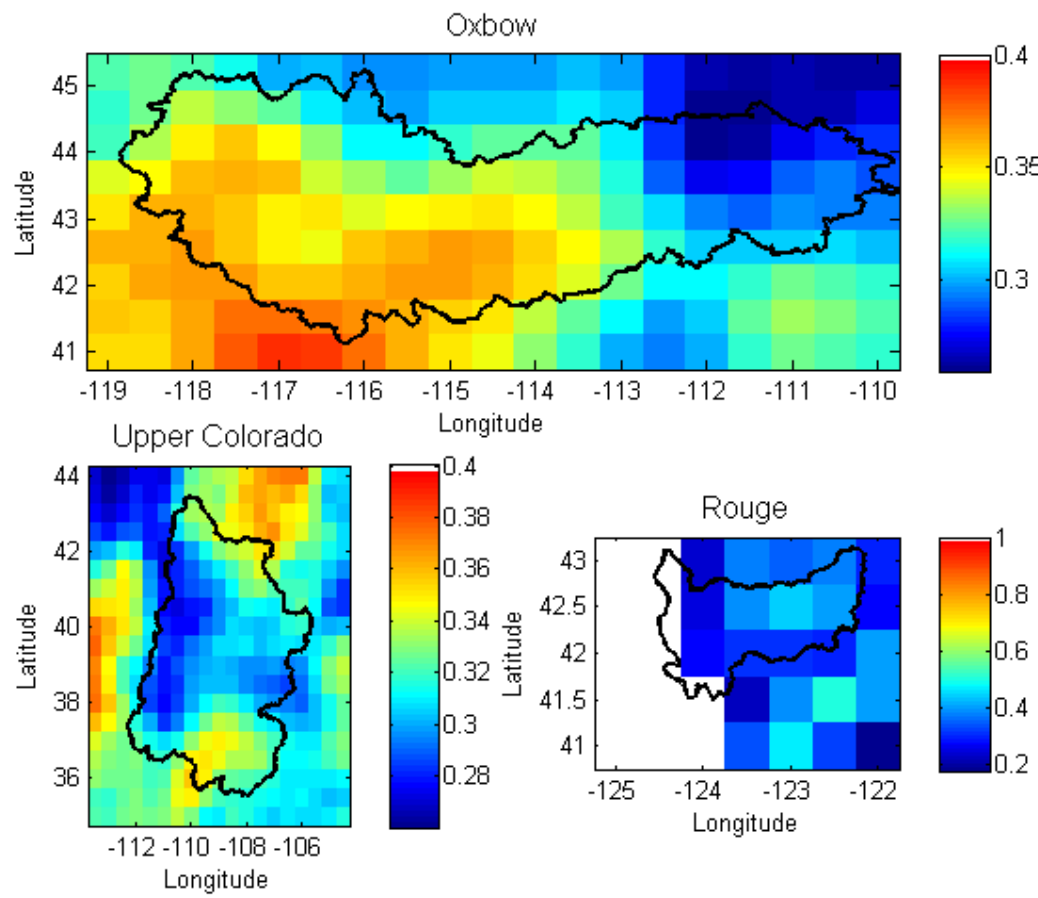

Figure 7. KGE measure calculated between the observation and raw forecast for monthly precipitation over the calibration period (1979-1999) for the study basin, the white grid cells in Rouge River basin are the ones with missing observation.
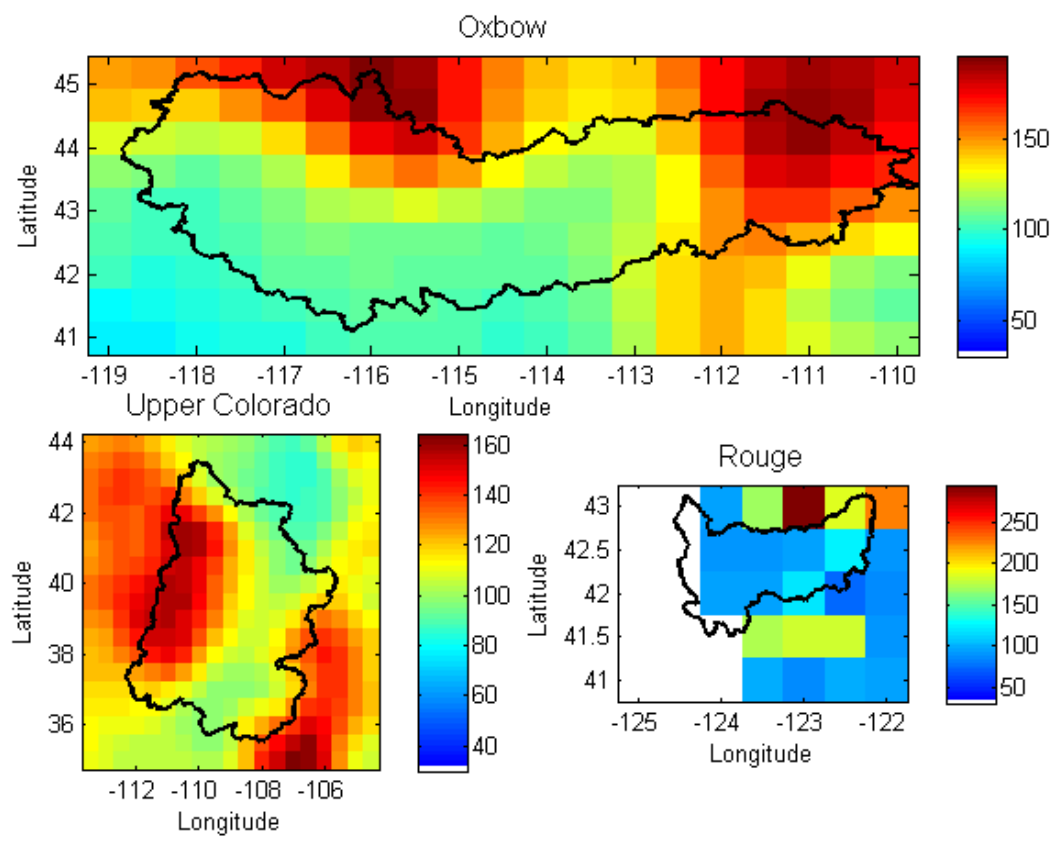

Figure 8 .RMSE calculated in mm between the observation and raw forecast for monthly precipitation over the calibration period (1979-1999) for the study basin, the white grid cells in Rouge River basin are the ones with missing observation. 


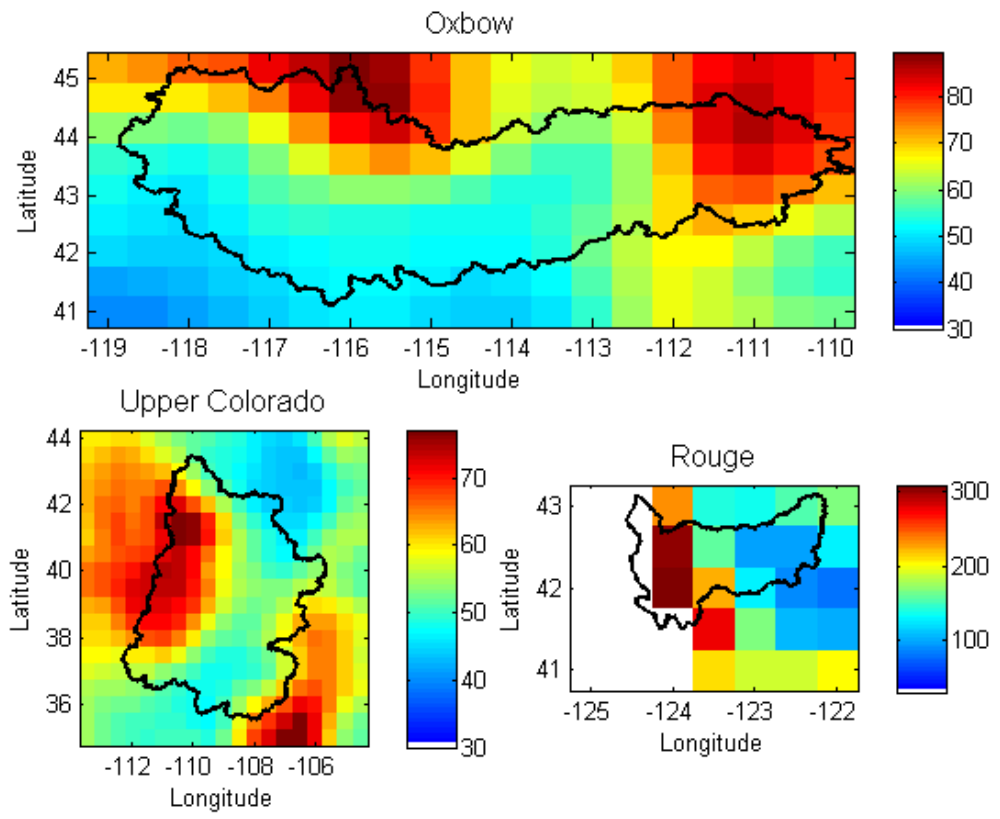

Figure 9 . MAE calculated in mm between the observation and raw forecast for monthly precipitation over the calibration period (1979-1999) for the study basin, the white grid cells in Rouge River basin are the ones with missing observation.

It can be concluded from the figures that raw forecast performance in predicting the precipitation over the selected basins is varied. To be more specific, in the NSE framework the forecast predicts negative values deducing that the observation mean is predicting better than the forecast. Same can be deduced from KGE, where most of the values are between $0.2-0.4$, that forecast is relatively underperforming as compared to observations. For MAE and RMSE, all the basins are showing relatively high errors in the forecast. Thus, there is need of bias correction method, in this case we are utilizing statistical EPP to achieve the same. 


\subsection{Designed Experiment}

Experiment consists of two phases, calibration and validation. In the calibration period the methodology described in chapter 2 is applied to the datasets, thus deducing transformation function needed for post-processing the future forecast. The corresponding transformation is further applied to the forecast in the validation period to verify the reliability of the technique. Figure 10 demonstrates the designed Experiment.

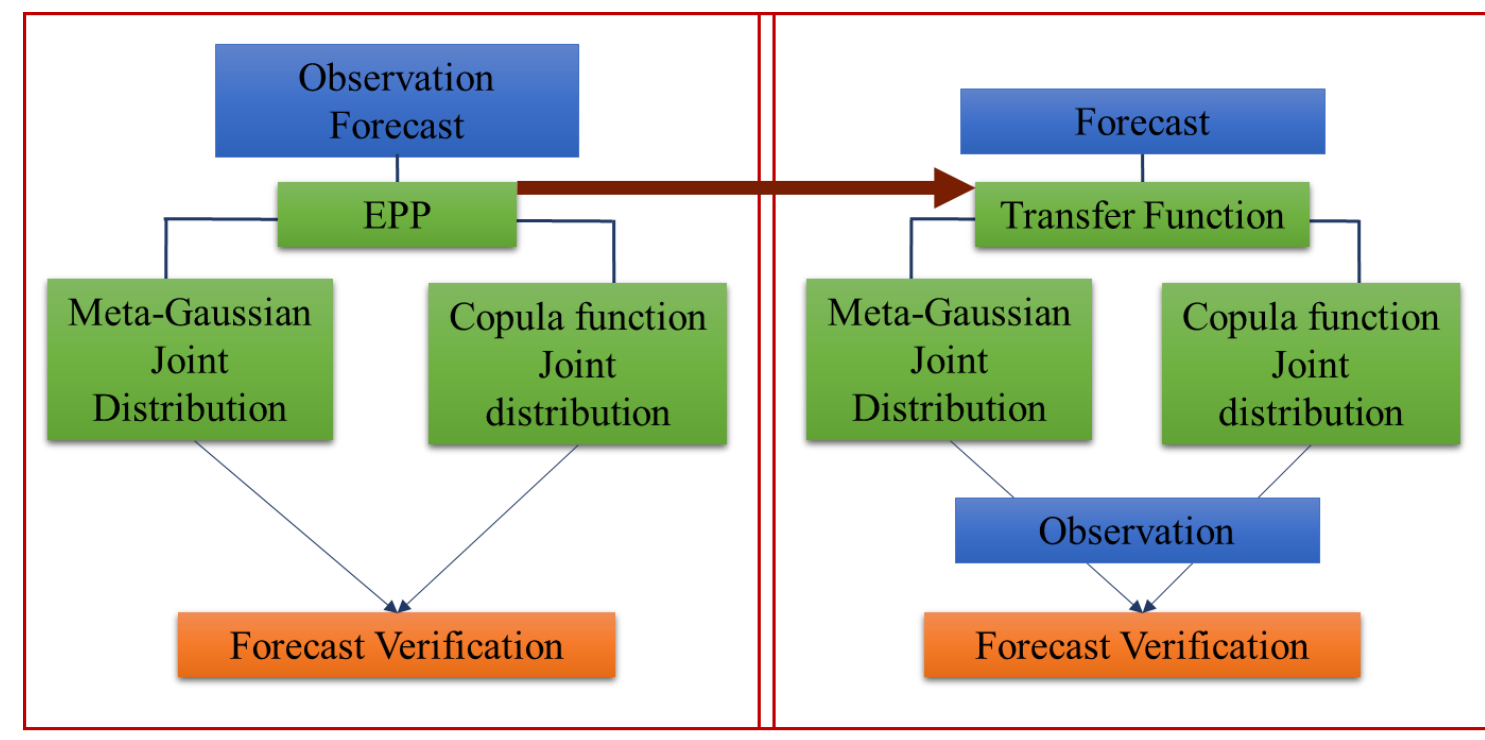

\section{Calibration}

\section{Validation}

Figure 10. Designed experiment for this study.

\subsubsection{Marginal Distribution}

Best marginal distribution that can describe the observation and forecast for each month is selected foremost. Results are evaluated against the performance of Weibull, Gamma, Exponential, and lognormal distribution in both K-S and AIC tests.. Results of 
combined performance of distribution on those tests are depicted in figure (11) to figure (16) for Colorado, Oxbow, and Rouge sub-basin observation and forecast, respectively.
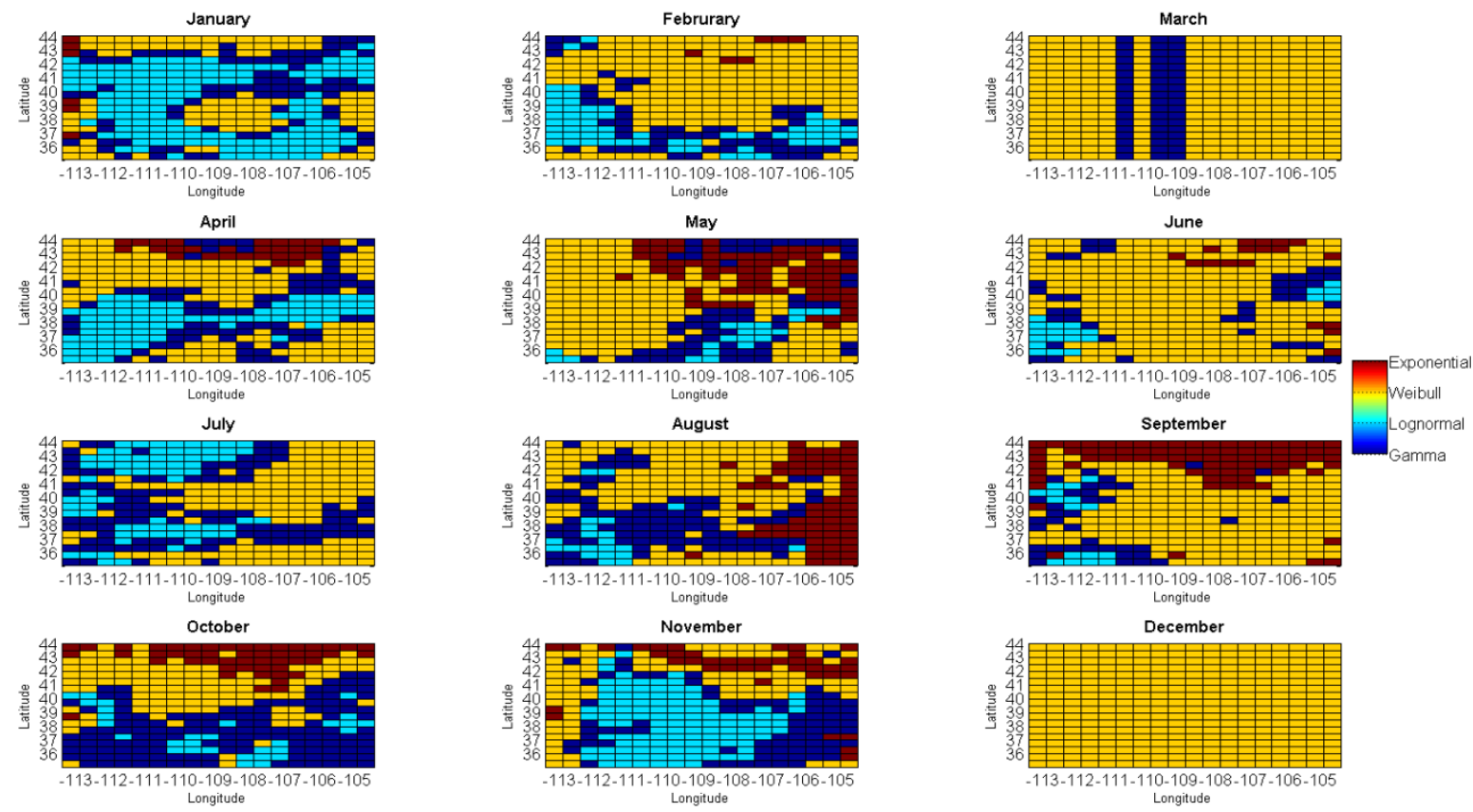

November
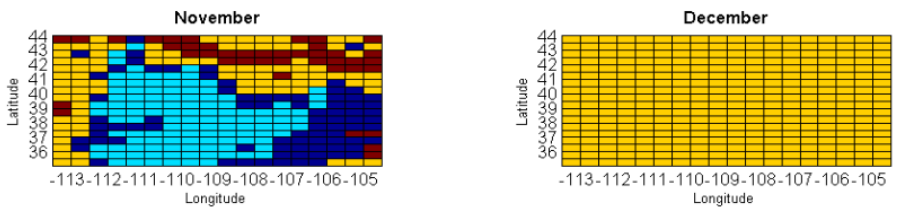

Figure 11 .Fitted marginal distribution to monthly historical observation (1979-1999) over Colorado River basin. The marginal distributions were chosen among Exponential, Weibull, Lognormal, and Gamma. 

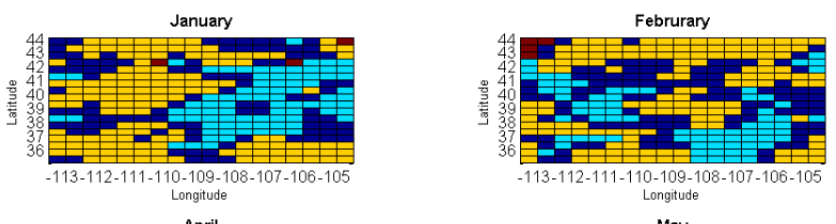

May

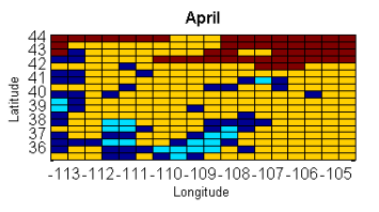

July

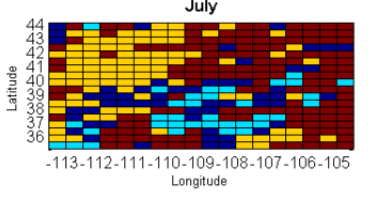

October

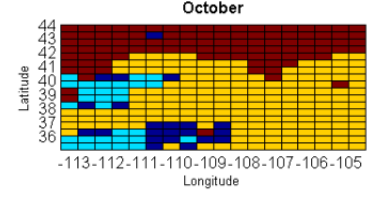

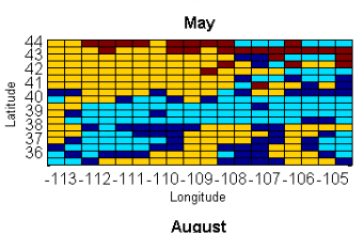

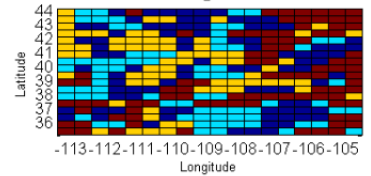

November

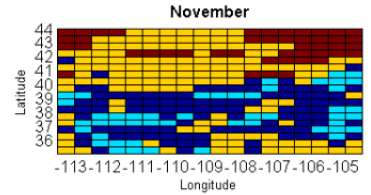

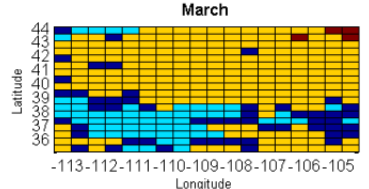

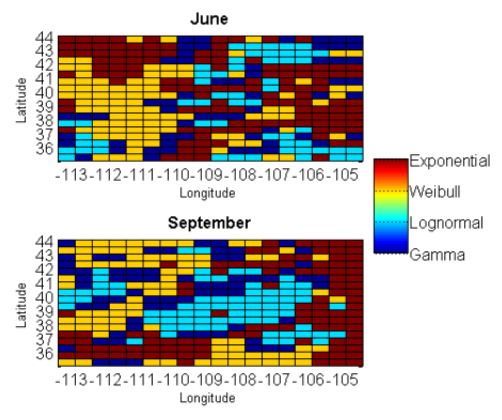

December

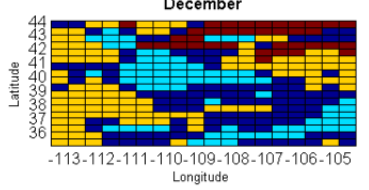

Figure 12 . Fitted marginal distribution to monthly historical forecast (1979-1999) over Colorado River basin. The marginal distributions were chosen among Exponential, Weibull, Lognormal, and Gamma.
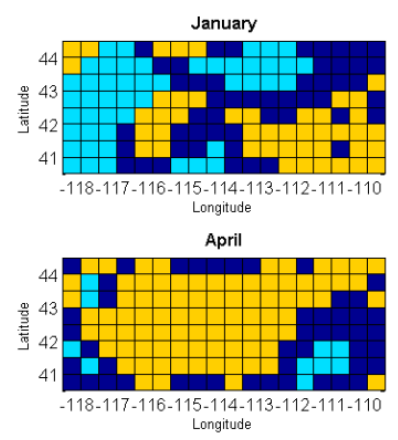

July

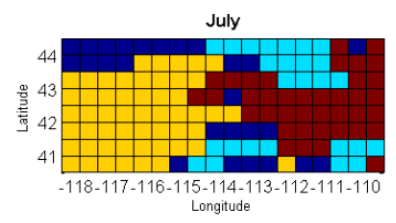

Longitude

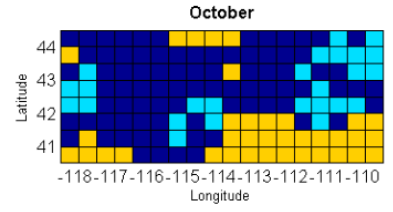

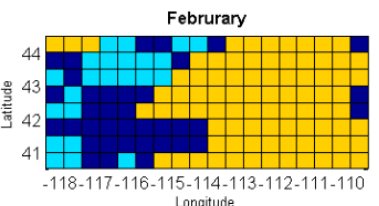

May

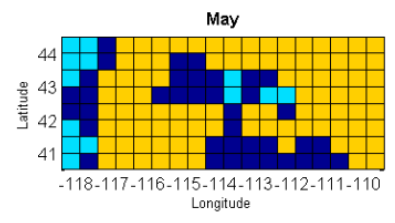

August

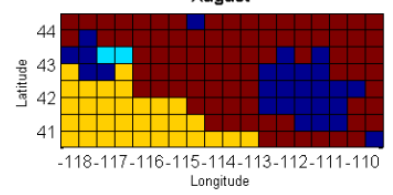

November

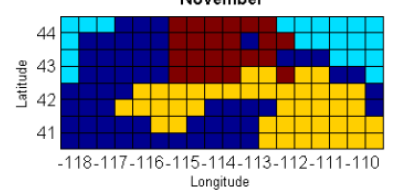

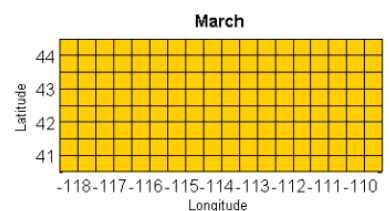

June

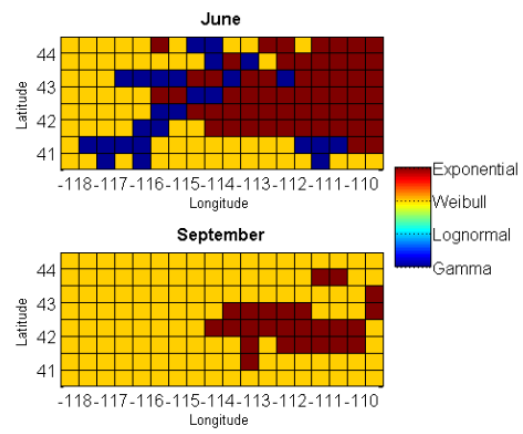

December

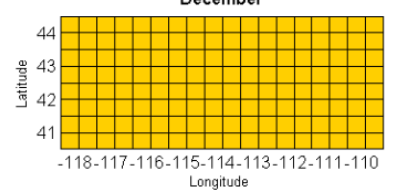

Figure 13 . Fitted marginal distribution to monthly historical observation (1979-1999) over Oxbow River basin. The marginal distributions were chosen among Exponential, Weibull, Lognormal, and Gamma. 

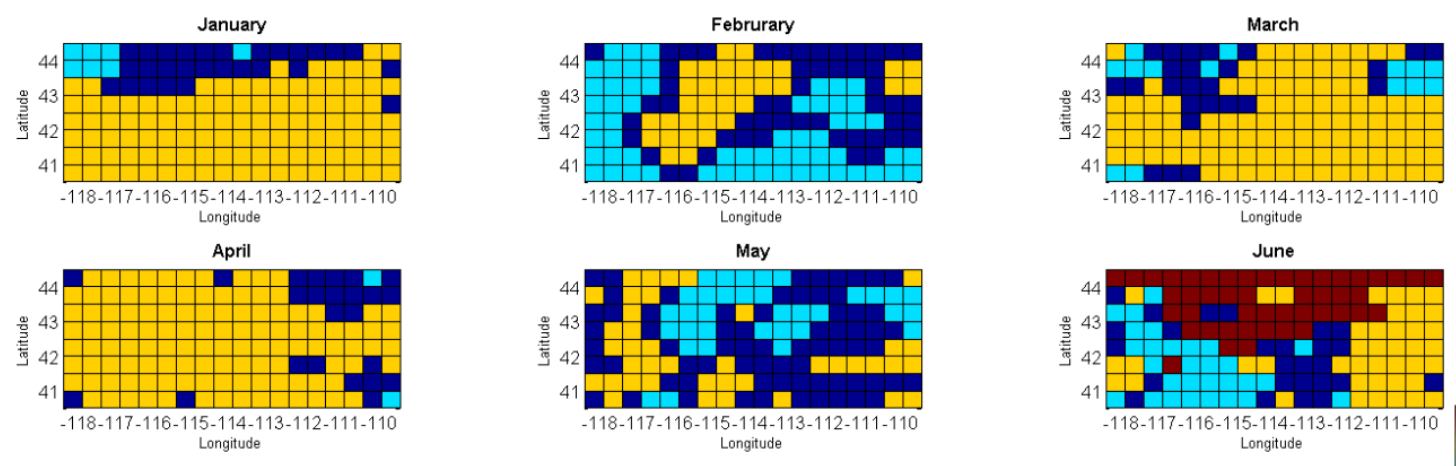

May
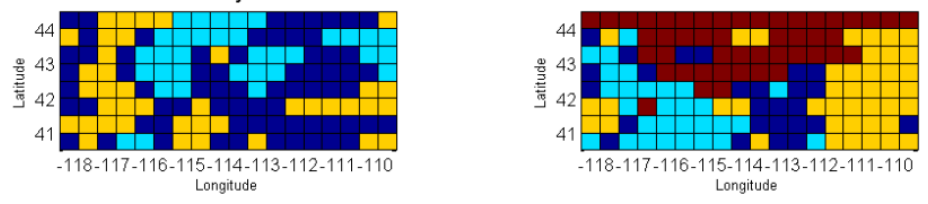

August
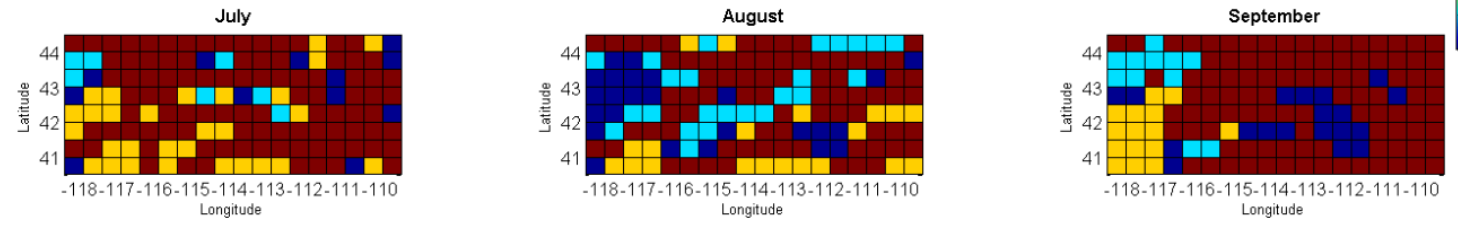
Longitude
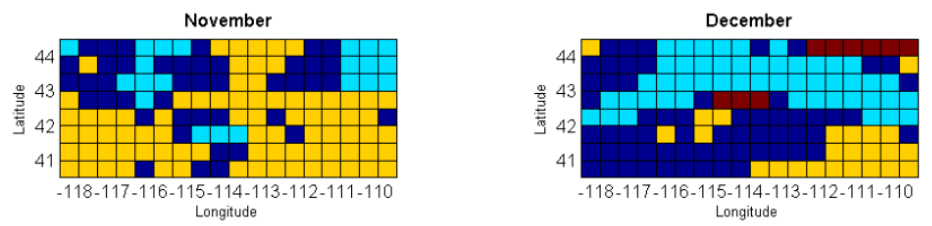

Figure 14 .Fitted marginal distribution to monthly historical forecast (1979-1999) over Oxbow River basin. The marginal distributions were chosen among Exponential, Weibull, Lognormal, and Gamma.

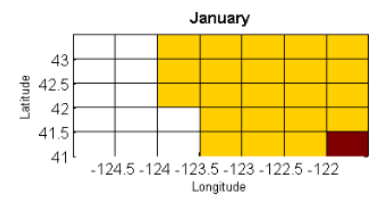

April

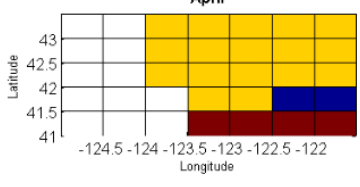

July

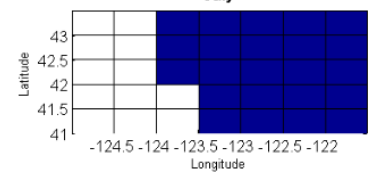

October

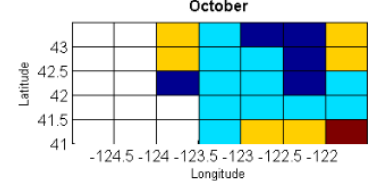

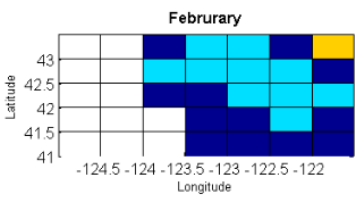

May

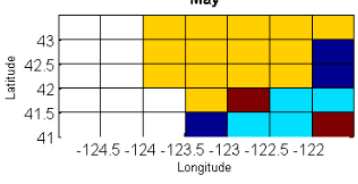

August

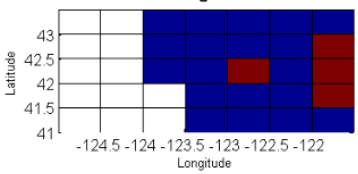

November

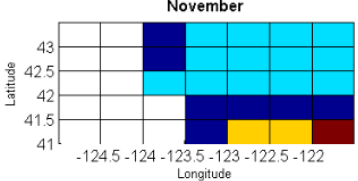

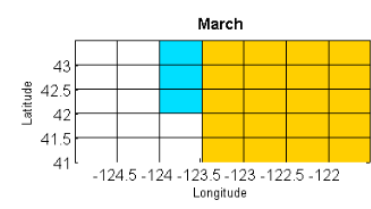

June

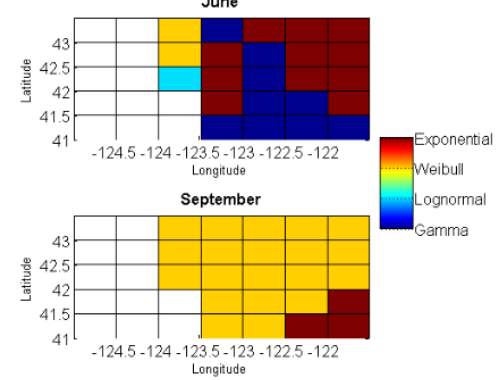

December

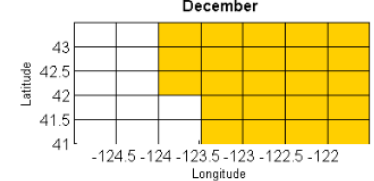

Figure 15 .Fitted marginal distribution to monthly historical observation (1979-1999) over Rouge River basin. The marginal distributions were chosen among Exponential, Weibull, Lognormal, and Gamma. 

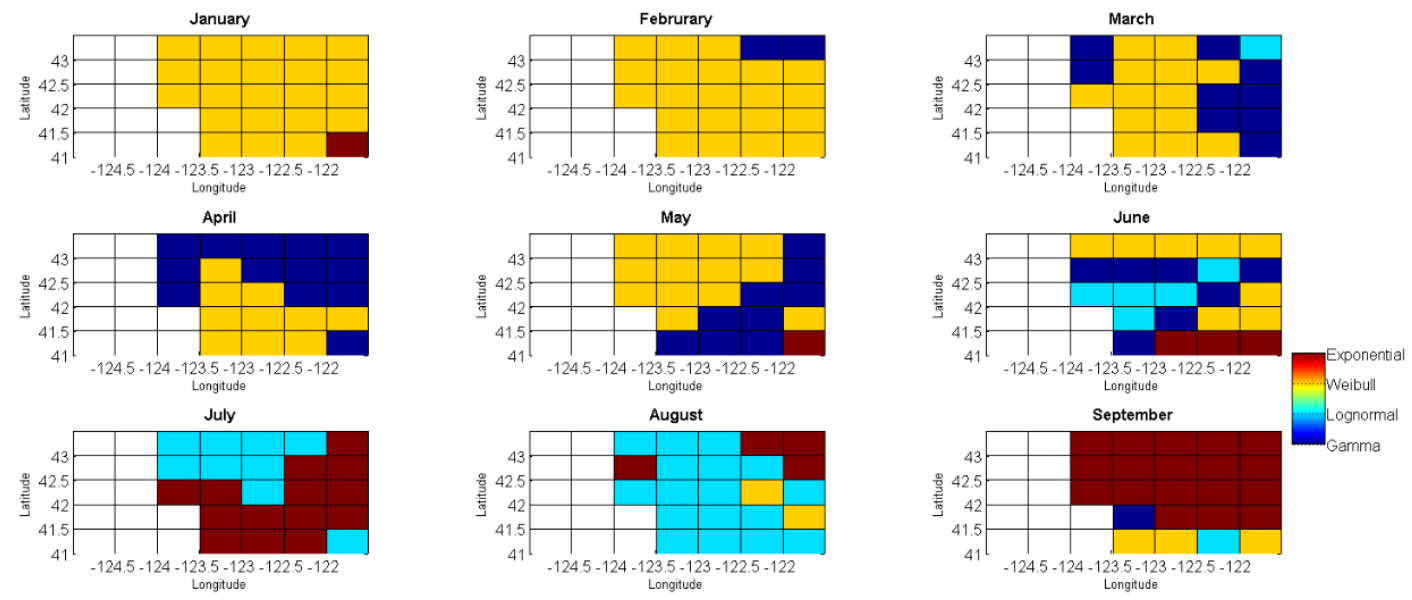

November
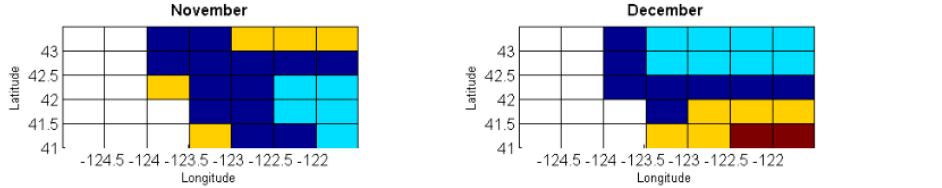

Figure 16 .Fitted marginal distribution to monthly historical forecast (1979-1999) over Rouge River basin. The marginal distributions were chosen among Exponential, Weibull, Lognormal, and Gamma.

It can be noted from the figures that the behaviors of the raw forecast and observation are different, as was pointed out in figure 6-9. Interestingly, months with extreme precipitation amount e.g. March and December, Weibull distribution is appropriate for describing the distribution of observation over all the basins. Accordingly, this points towards consistency in observation dataset. On the other hand, the forecast data doesn't show a specific pattern for the fitted distribution to the monthly precipitation. There is large variation across temporal and spatial scales in all the sub-basins for selected marginal distribution and no specific pattern could be pointed towards. 


\subsubsection{Copula Functions}

Similarly, as above section, the best copula function that can describe the joint distribution between the observation and forecast in the historical period is chosen using GoF. The test statistics are calculated using observation and forecast on all spatio-temporal scales in all the sub-basins. Figure (17) to (19) depicts the copula function chosen for each cell for different months over the study basins with GoF statistics.

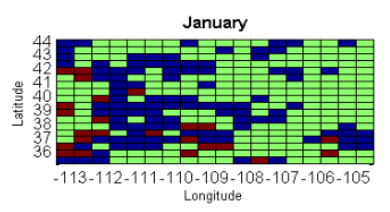

April

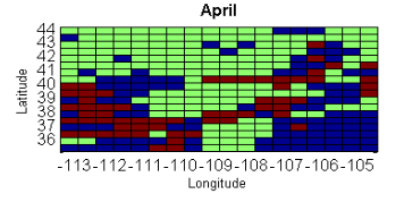

July
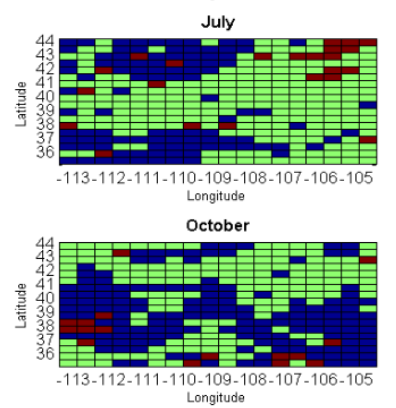

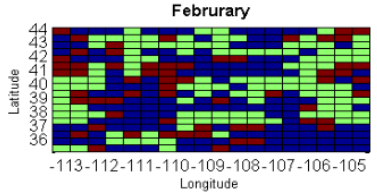

May

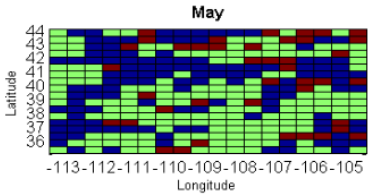

August
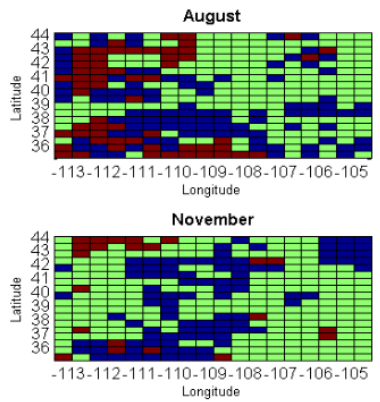

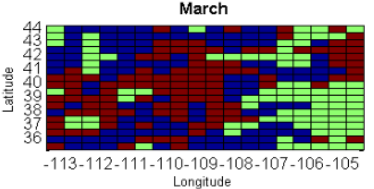

June

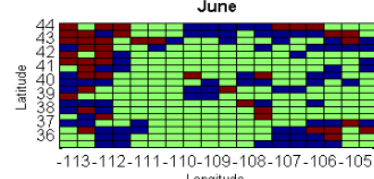
September

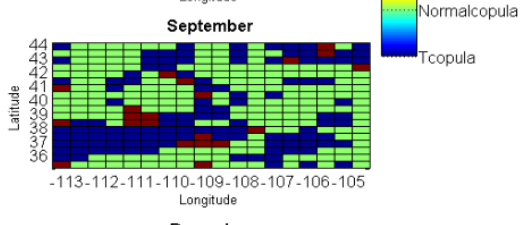

December

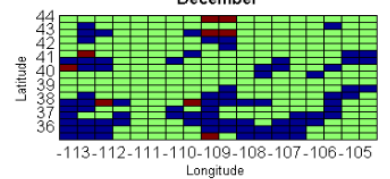

Figure 17 . Selected copula functions among for each grid cell over Upper Colorado River Basin. Frank copula, normal copula, and t copula are shown in red, green, and blue 

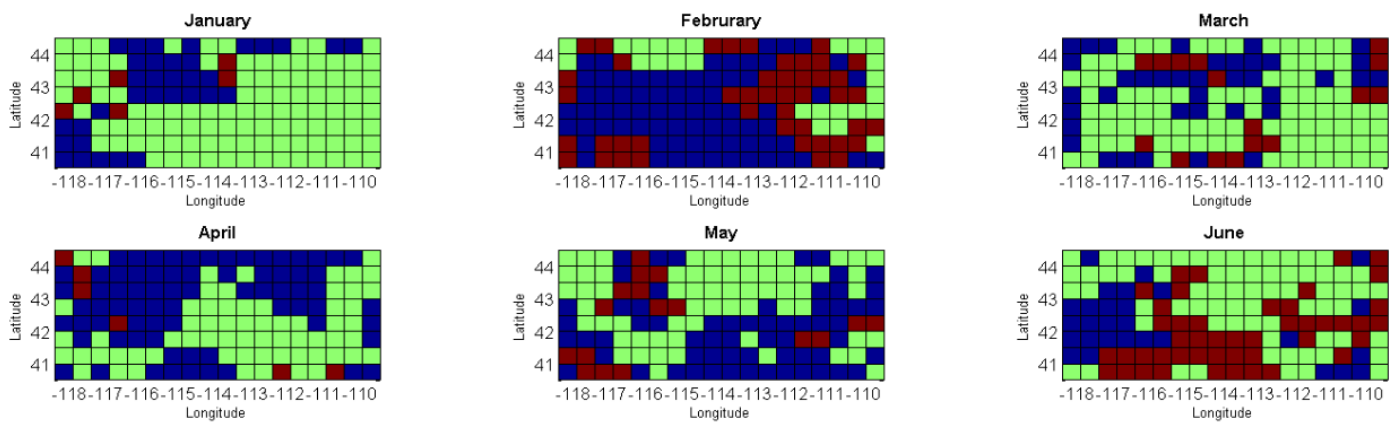

August
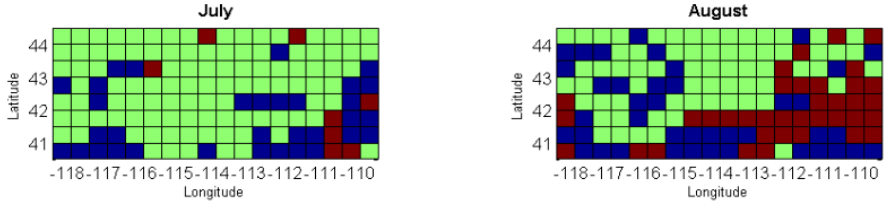

ongitude

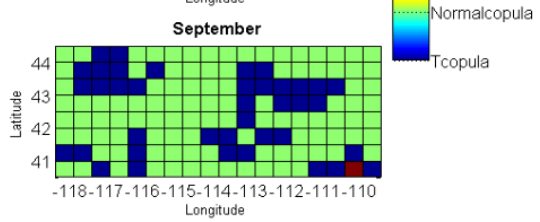

November
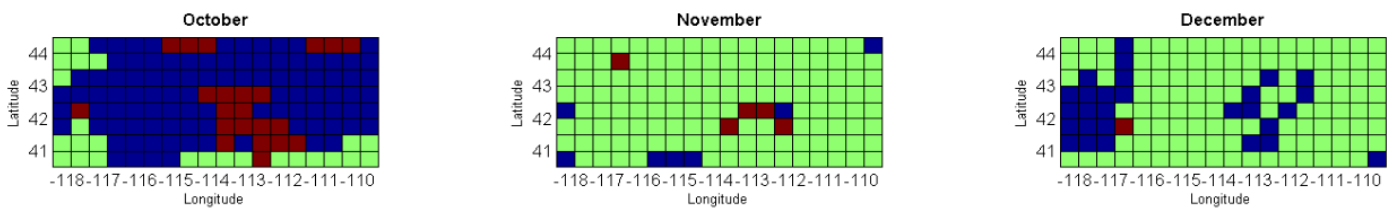

Figure 18 . Selected copula functions among for each grid cell over Oxbow River Basin. Frank copula, normal copula, and t copula are shown in red, green, and blue

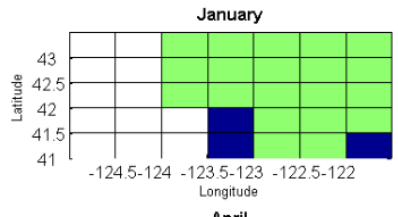

April

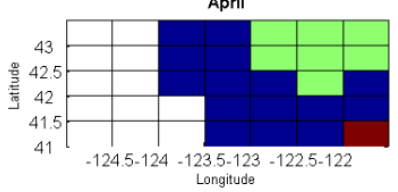

July

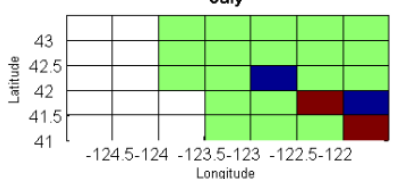

October

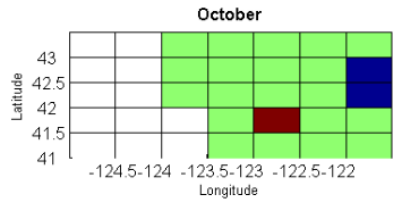

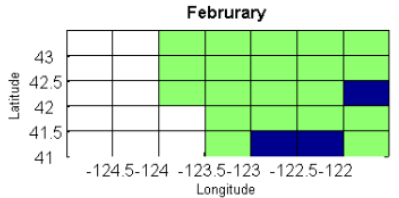

May
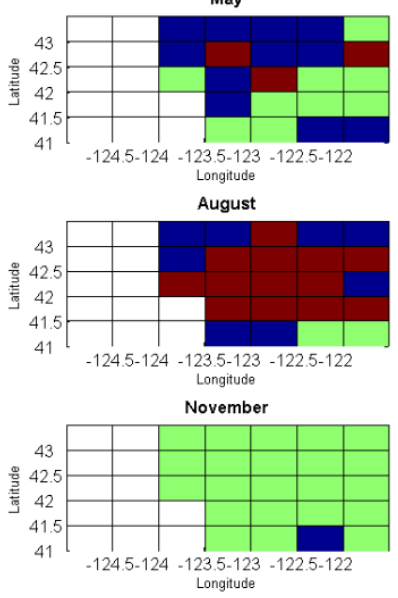

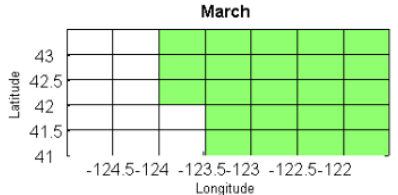

June
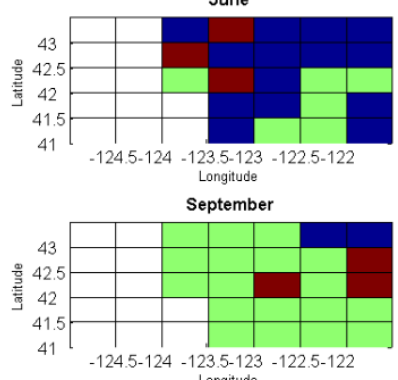

December

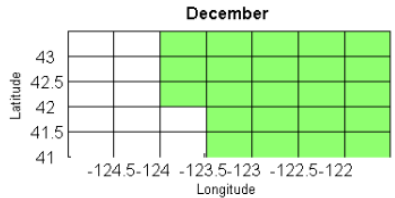

Figure 19. Selected copula functions among for each grid cell over Rouge River Basin. Frank copula, normal copula, and t copula are shown in red, green, and blue 
It should be noted that among all the copula functions, Normal copula has emerges to be most powerful in describing the joint distribution between the observation and forecast in most of the cells. Upper Colorado basin presents most spatio-temporal diversity for chosen copulas followed by Oxbow and Rouge. This could be attributed to size of the sub-basin wherein larger ones provides greater diversity and thus varied distribution. Information from the above chosen copula functions assists in building the ensemble precipitation forecast for each cell in each of the basins on monthly scales. In this study, we target to build 20 ensemble members of the precipitation forecast.

\subsubsection{Forecast Verification Results}

Performing EPP with two diverse methods provides users added advantage of evaluation of strengths/weakness of the proposed methodologies. It also helps with investigation of reliability of the generated ensemble forecast through the verification measures described above. In the following sections, reliability will be assessed through

the deterministic measures to evaluate the ensemble mean behavior followed by probabilistic measures. The results are divided into calibration and verification periods, to investigate the procedure's capability to predict future precipitation forecast.

\subsubsection{Deterministic Forecast Verification}

The results are evaluated with four measures namely MAE, RMSE, KGE, and NSE. For the first two measures, since they are dependent on the length of the dataset, it would be inappropriate to compare the calibration and verification periods with themselves but comparison with raw forecast could be done for both the calibration and validation period. 
Results for MAE are highlighted in figures (20) through (25) representing MAE in calibration and validation period, respectively.

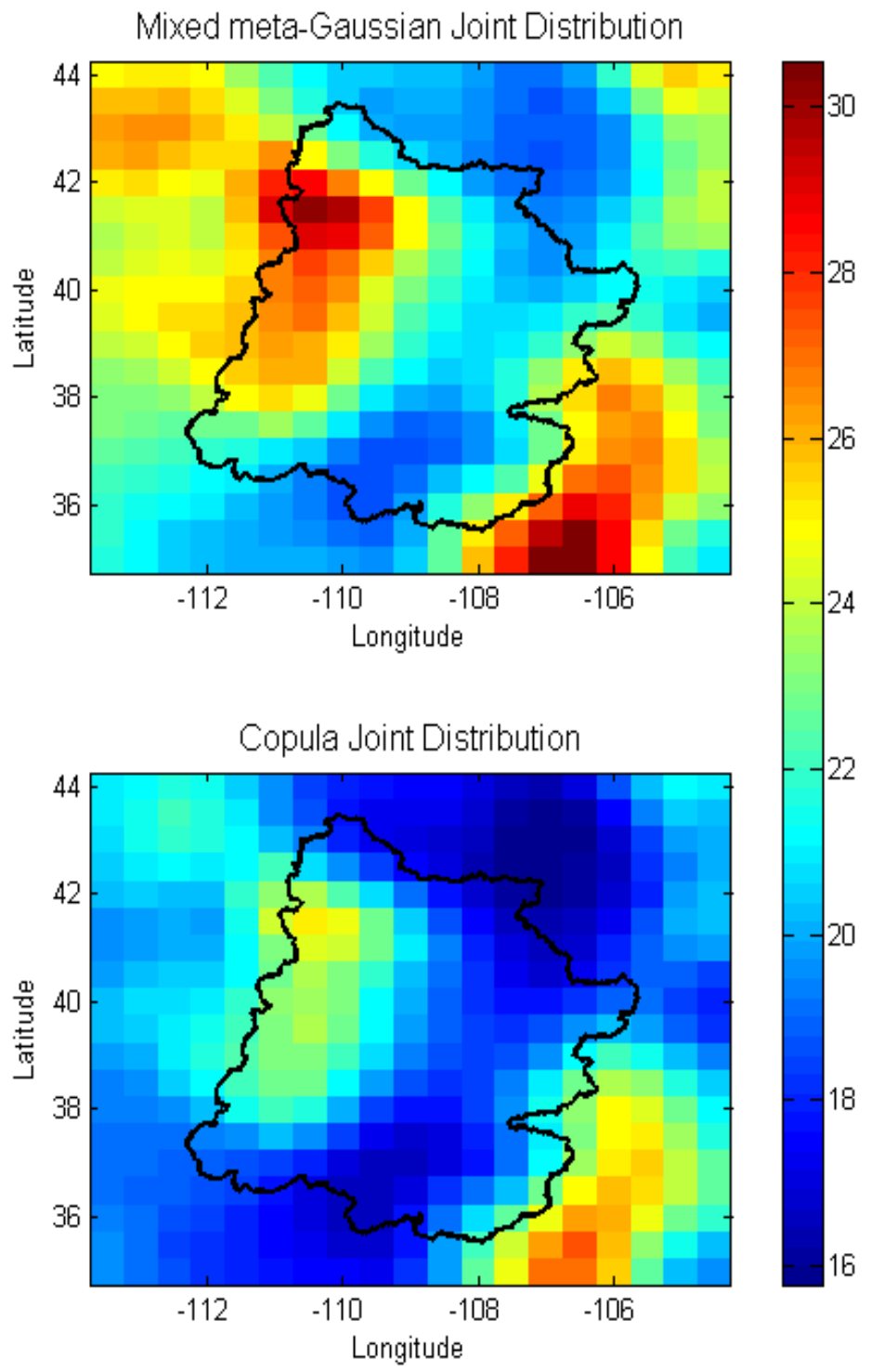

Figure 20.Calculated MAE (mm) over Upper Colorado River Basin for two diverse generated mean precipitation ensemble forecast during calibration period. meta-Gaussian approach (top) and copula approach (bottom) 
In Upper Colorado River basin, MAE in the calibration period shows a significant improvement of almost $50 \%$ in comparison to the raw forecast in both the applied methodologies. However, EPP based on the copula functions shows fewer errors in the generated mean ensemble. It would be important to mention that the spatial pattern of the error in both of the techniques follows similar pattern. Similarly, for Oxbow and Rouge the forecast after EPP shows significant improvements as compared to raw forecast. Similar conclusion can be drawn about copula based EPP, which presents better improvements compared to other method in both the basins. When comparing the basins' results, it is distinguishable that basin with higher precipitation amount (irrespective of size of basin) have higher range of the errors, for instance, Rouge has almost up to 5 times of the errors as compared to Oxbow and UCRB. 


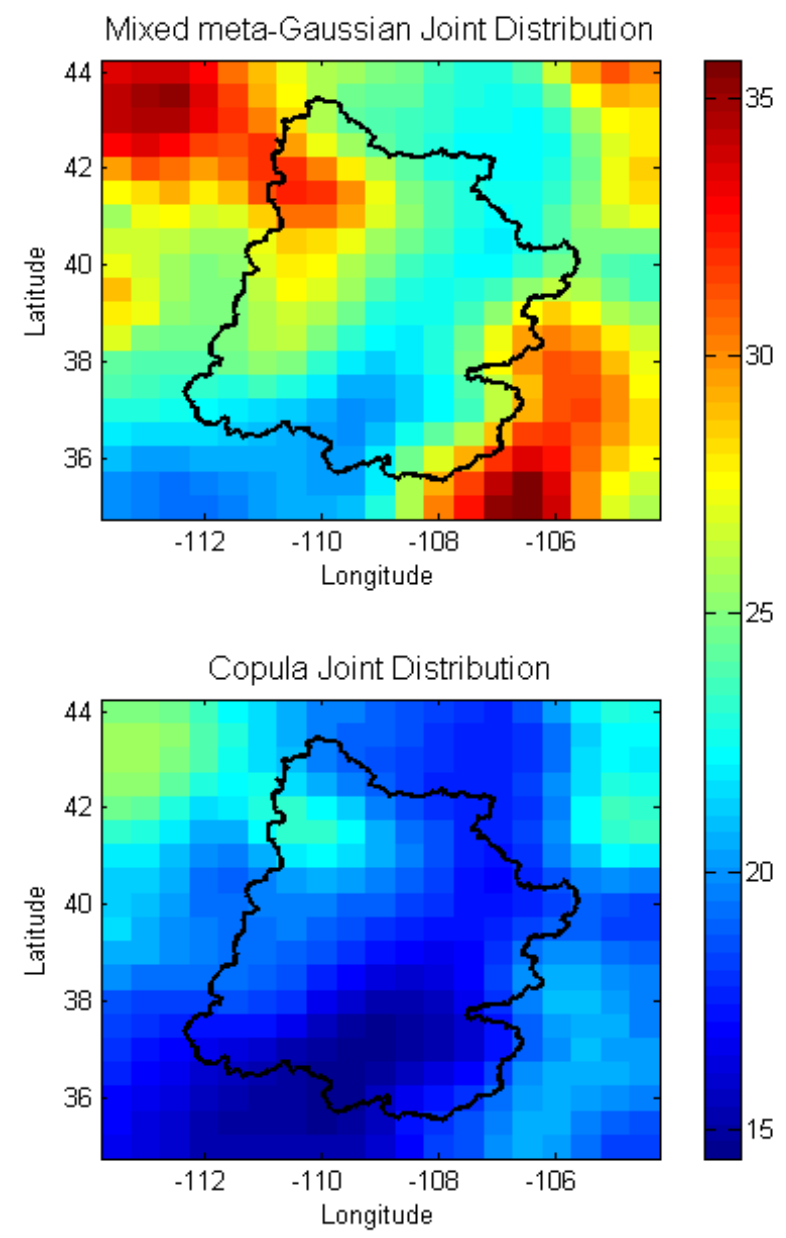

Figure 21. Calculated MAE (mm) over Upper Colorado River Basin for two diverse generated mean precipitation ensemble forecast during validation period. Meta-Gaussian approach (top) and Copula approach (bottom) 


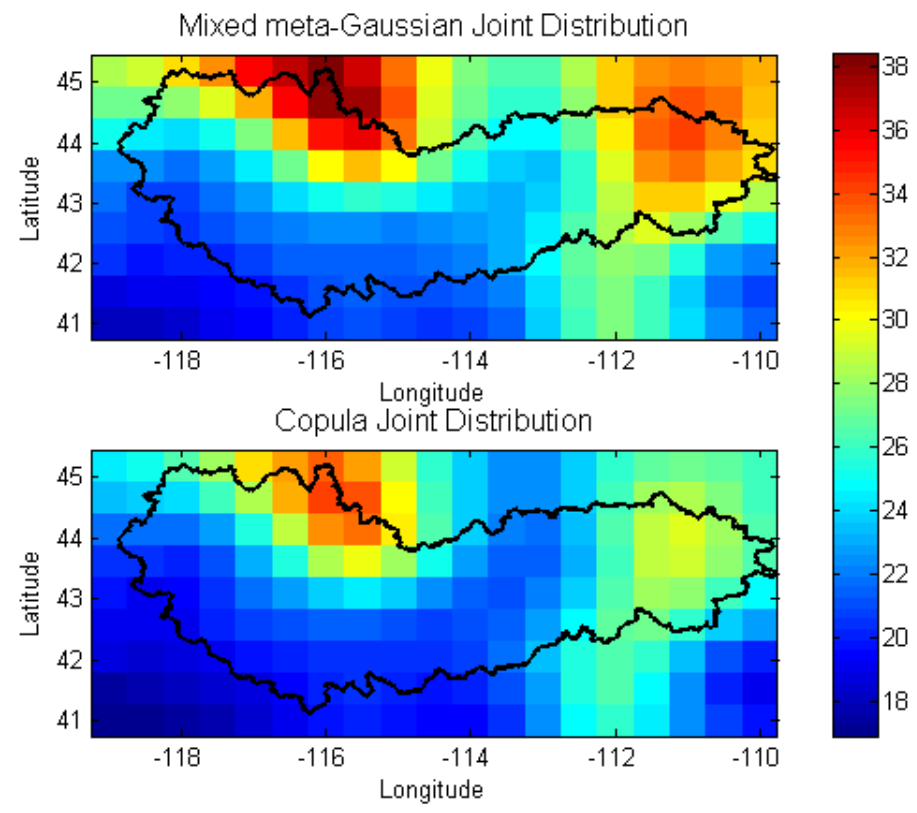

Figure 22 .Calculated MAE (mm) over Oxbow River Basin for two diverse generated mean precipitation ensemble forecast during calibration period. Meta-Gaussian approach (top) and Copula approach (bottom)

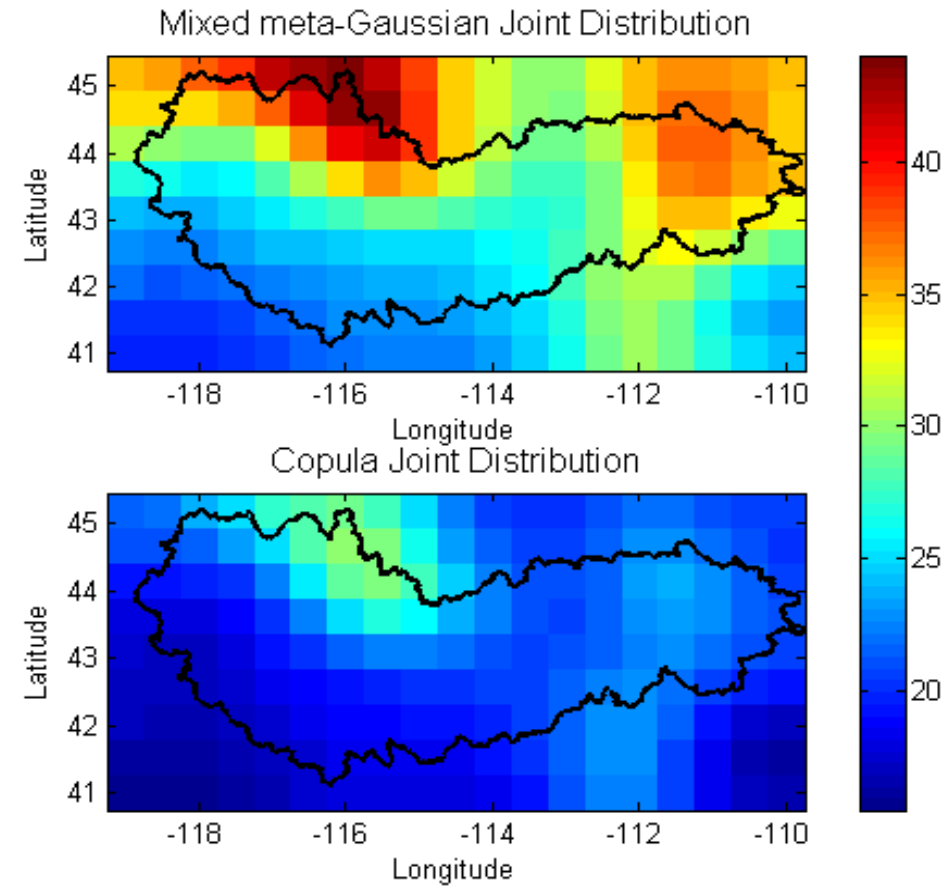

Figure 23.Calculated MAE (mm) over Oxbow River Basin for two diverse generated mean precipitation ensemble forecast during validation period. Meta-Gaussian approach (top) and Copula approach (bottom) 


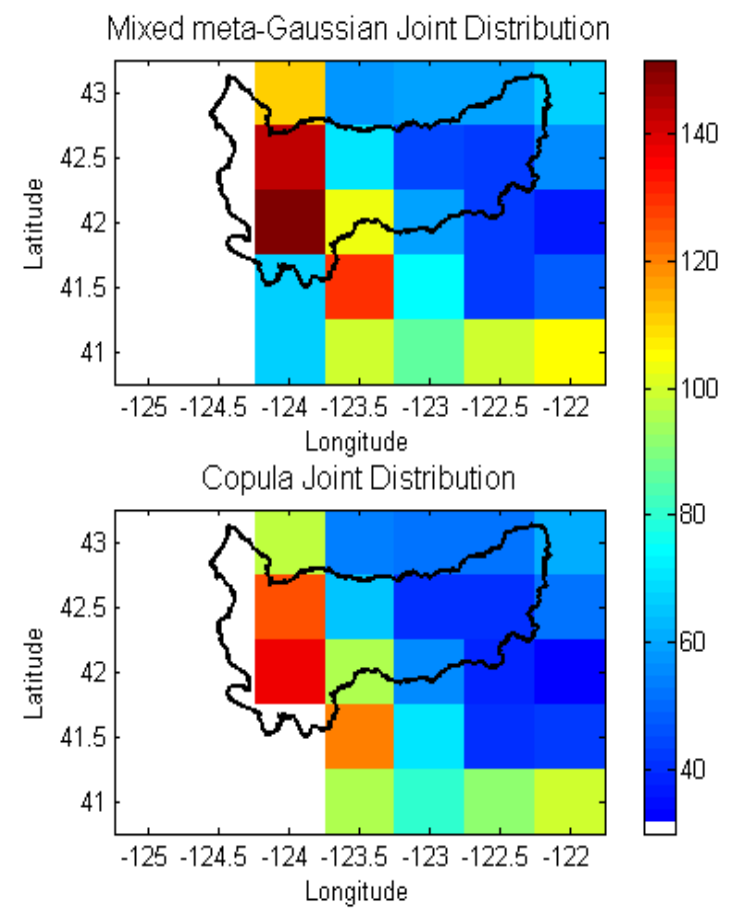

Figure 24. Calculated MAE (mm) over Rouge River Basin for two diverse generated mean precipitation ensemble forecast during calibration period. Meta-Gaussian approach (top) and Copula approach (bottom)

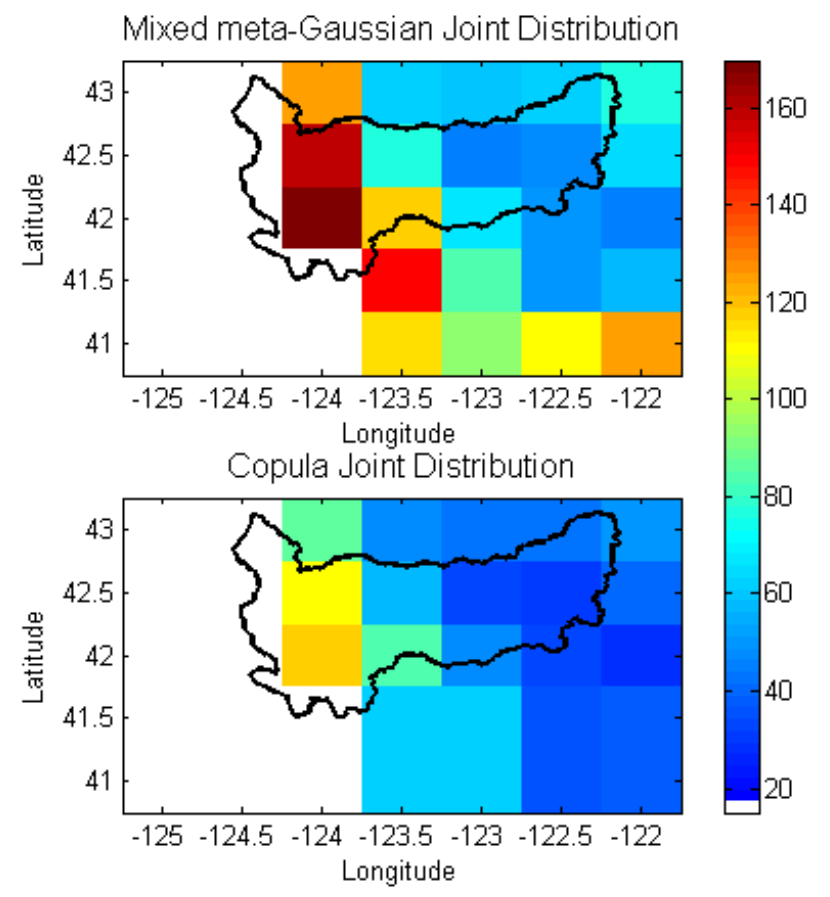

Figure 25. Calculated MAE ( $\mathrm{mm}$ ) over Rouge River Basin for two diverse generated mean precipitation ensemble forecast during validation period. Meta-Gaussian approach (top) and Copula approach (bottom) 


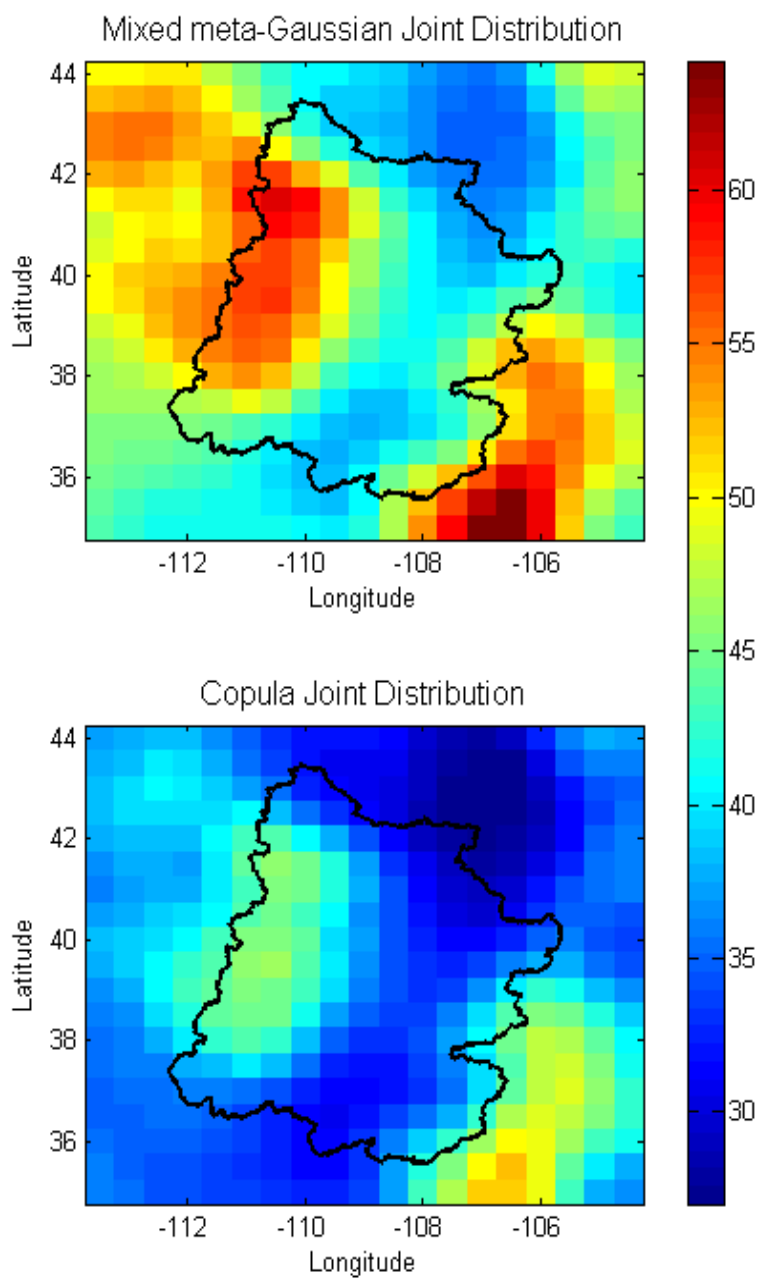

Figure 26 .Calculated RMSE ( $\mathrm{mm}$ ) over Upper Colorado River Basin for two diverse generated mean precipitation ensemble forecast during calibration period. Meta-Gaussian approach (top) and Copula approach (bottom) 


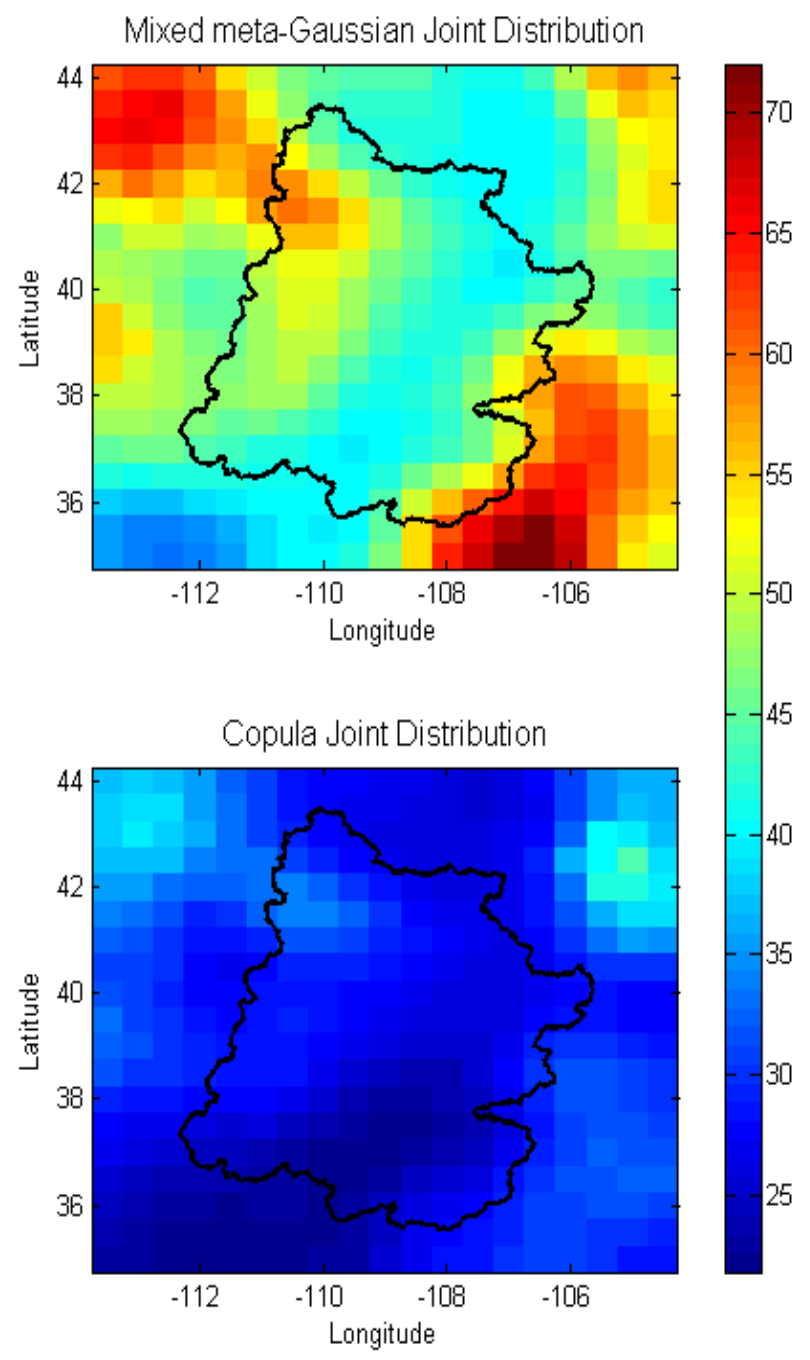

Figure 27. Calculated RMSE ( $\mathrm{mm}$ ) over Upper Colorado River Basin for two diverse generated mean precipitation ensemble forecast during validation period. Meta-Gaussian approach (top) and Copula approach (bottom)

RMSE results are presented in figures (26) through (31) representing RMSE in calibration and validation period, respectively. RMSE is more accurate in displaying errors in the forecast. It highlights the larger errors in the dataset by squaring them. Although the pattern/behavior of this measure over the generated and raw forecast is similar as was seen in MAE for all basins and the methods adopted for EPP. 


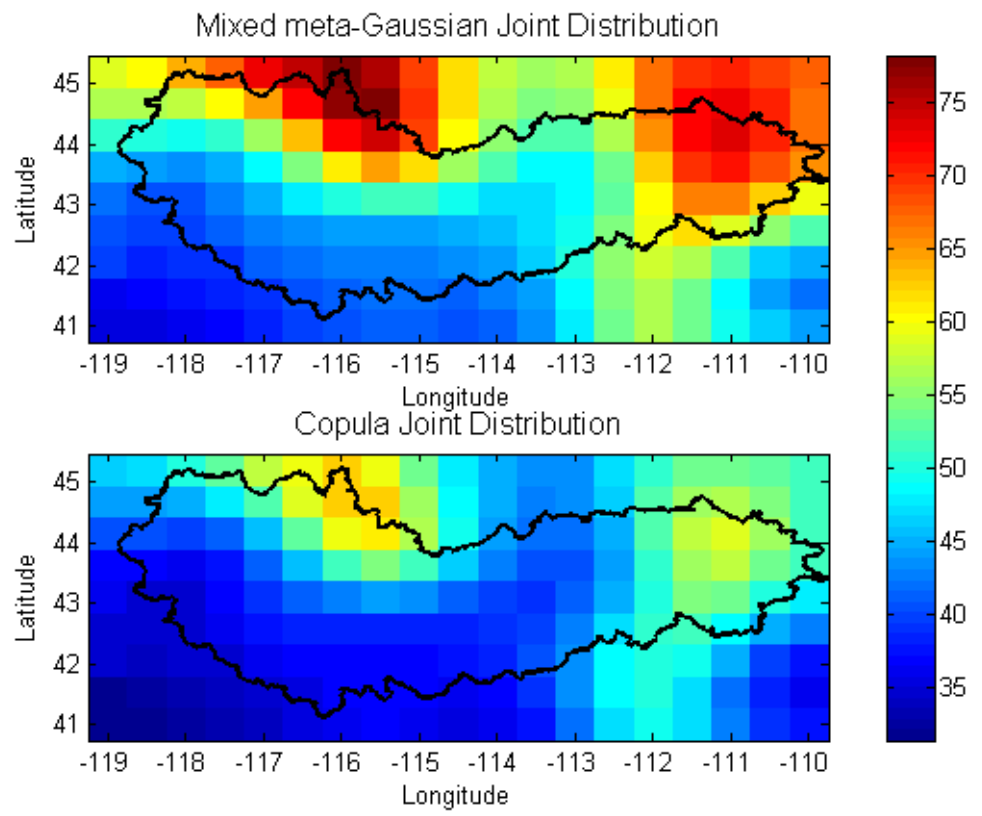

Figure 28. Calculated RMSE (mm) over Oxbow River Basin for two diverse generated mean precipitation ensemble forecast during calibration period. Meta-Gaussian approach (top) and Copula approach (bottom)

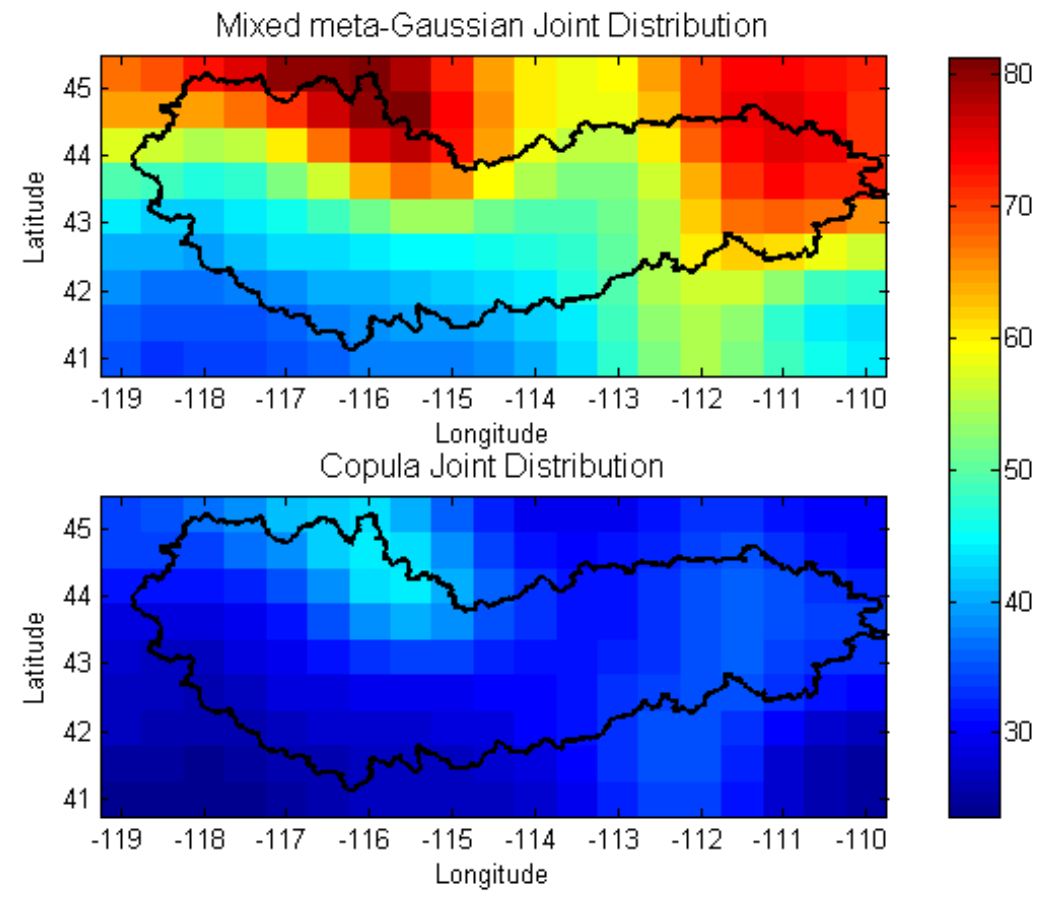

Figure 29. Calculated RMSE (mm) over Oxbow River Basin for two diverse generated mean precipitation ensemble forecast during validation period. Meta-Gaussian approach (top) and Copula approach (bottom) 


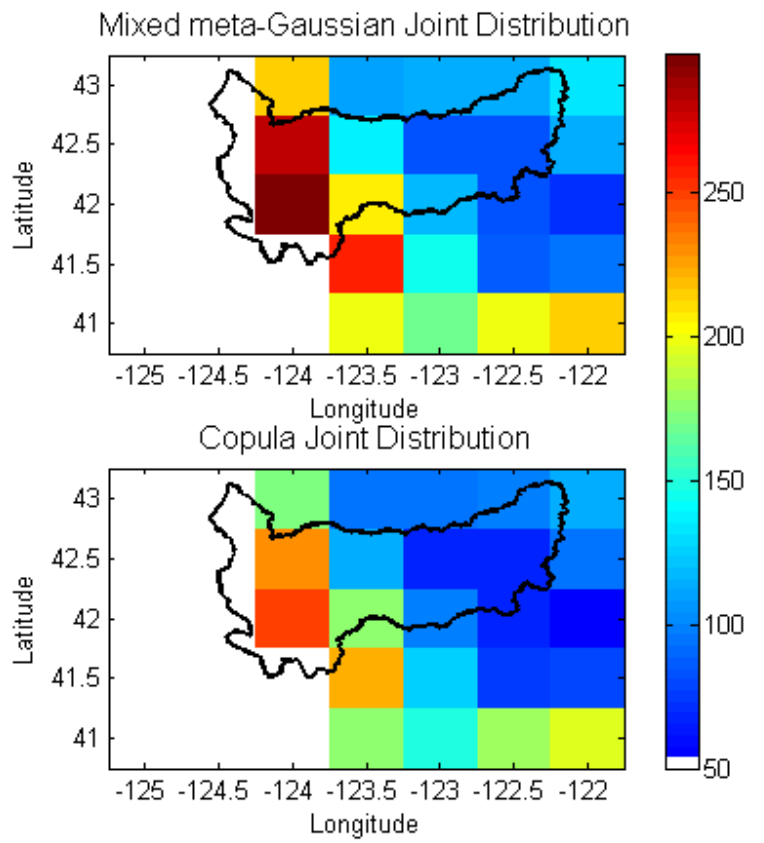

Figure 30. Calculated RMSE (mm) over Rouge River Basin for two diverse generated mean precipitation ensemble forecast during calibration period. Meta-Gaussian approach (top) and Copula approach (bottom)

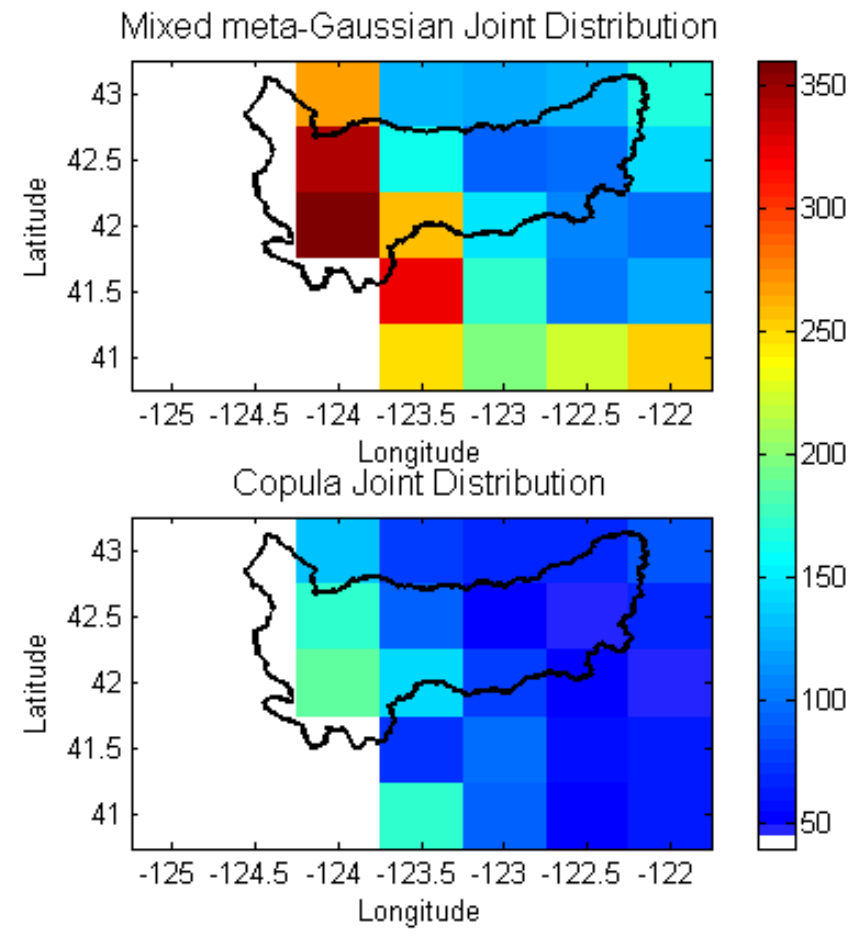

Figure 31 . Calculated RMSE (mm) over Rouge River Basin for two diverse generated mean precipitation ensemble forecast during validation period. Meta-Gaussian approach (top) and Copula approach (bottom) 
Whereas, in case of KGE and NSE we can have comparison between calibration and verification periods together, due to the normalized nature of the measures. Results of KGE are presented in figures (32) through (37) for calibration and validation period respectively. In calibration period, for all the study basins (figure 32, 34 and 36), it shows a significant improvement in the generated forecasts vs. the raw one. Copula shows more improvement of approximately $10 \%$ over the calibration period, when we compared MBG. However, for Rouge MBG shows slightly more improvement as compared to copula based EPP. 


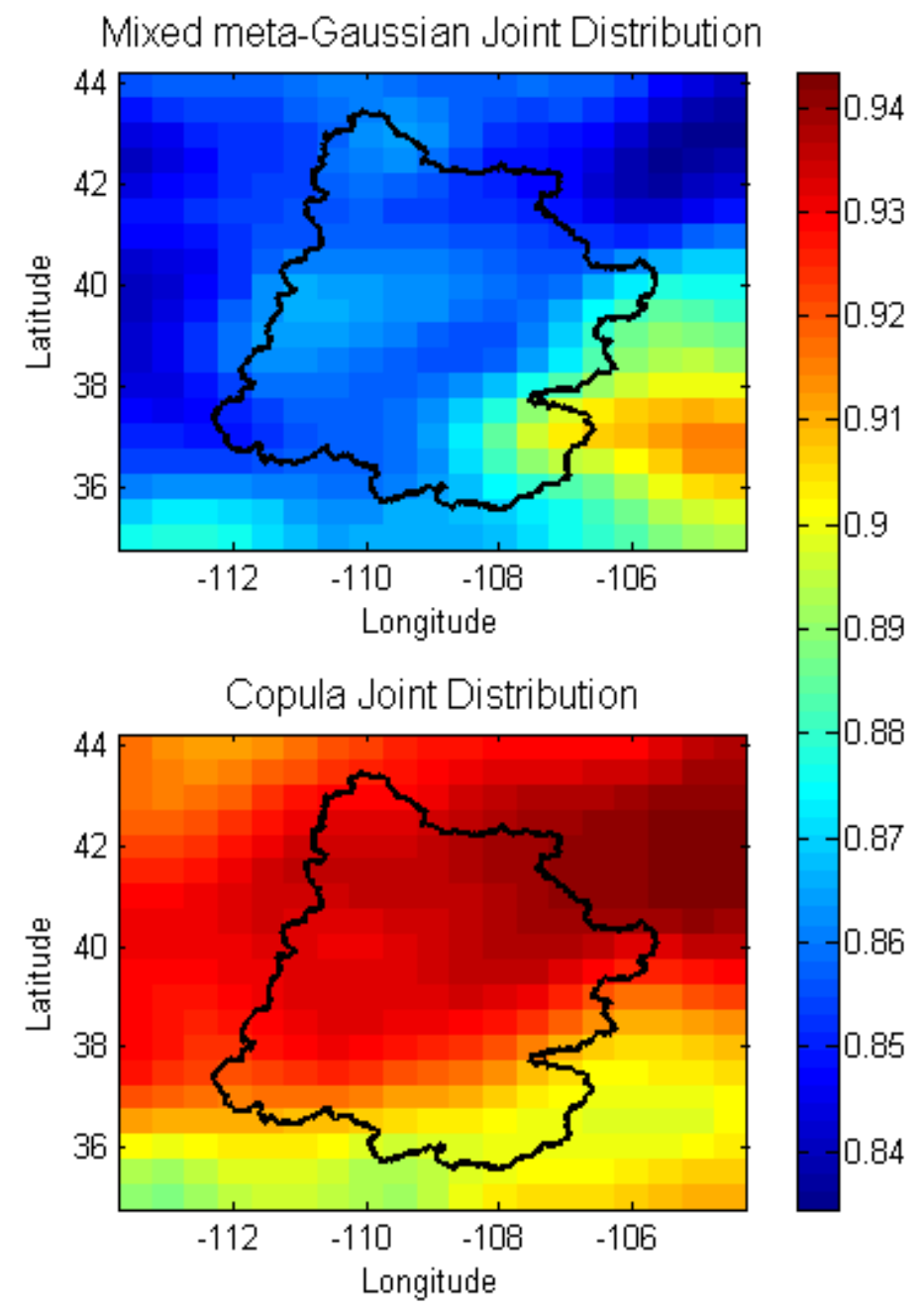

Figure 32. Calculated KGE over Upper Colorado River Basin for two diverse generated mean precipitation ensemble forecast during calibration period. Meta-Gaussian approach (top) and Copula approach (bottom)

Similarly during the validation period, for all the basins (figures 33, 35 and 37), copula based technique provides better improvements over MBG. It is interesting to note that in case of Rouge basin MBG fails to improve upon the results of calibration period. It is noteworthy to say that the copula function has less contraction in the validation part vs. the calibration period. 


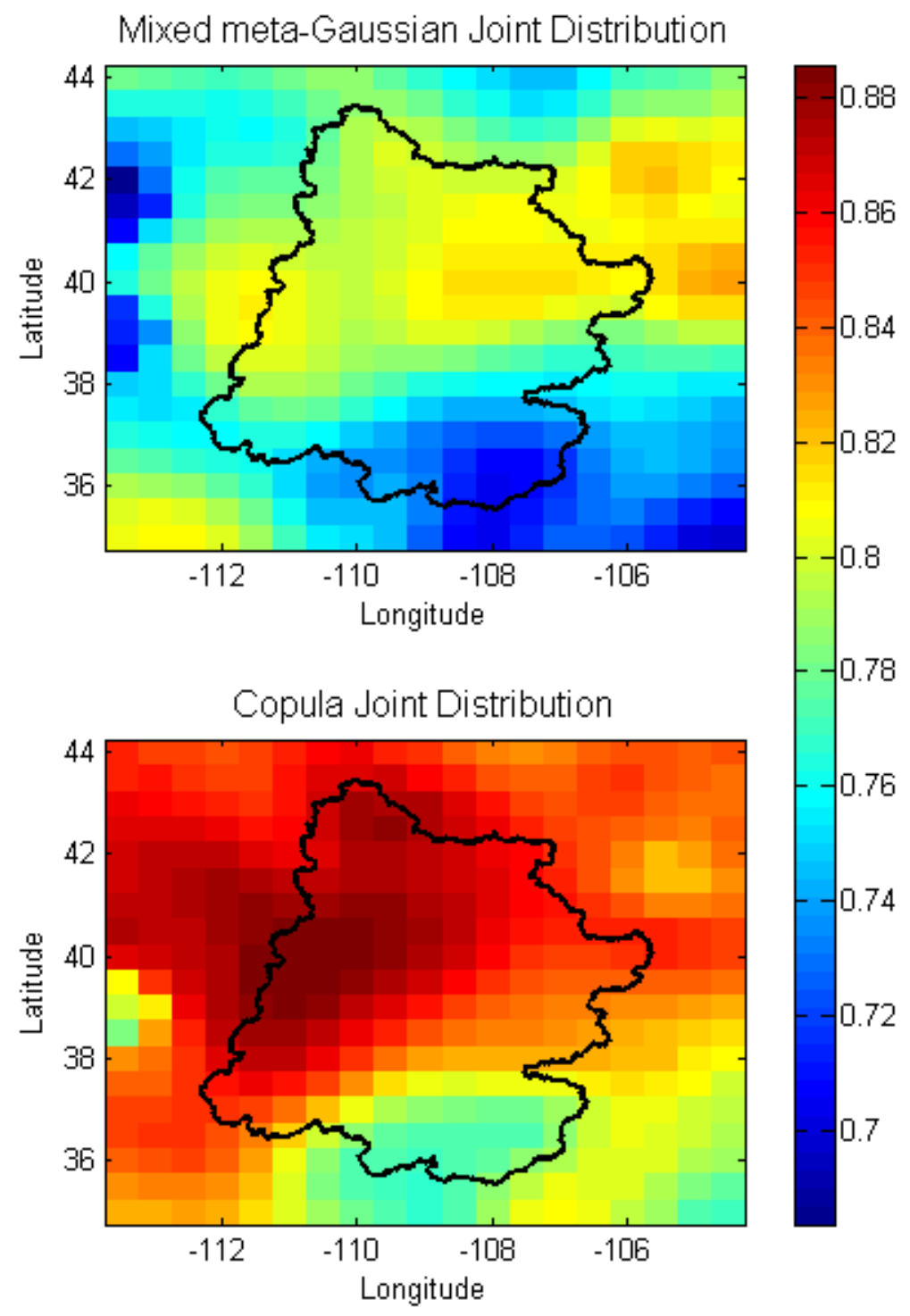

Figure 33. Calculated KGE over Upper Colorado River Basin for two diverse generated mean precipitation ensemble forecast during validation period. Meta-Gaussian approach (top) and Copula approach (bottom) 


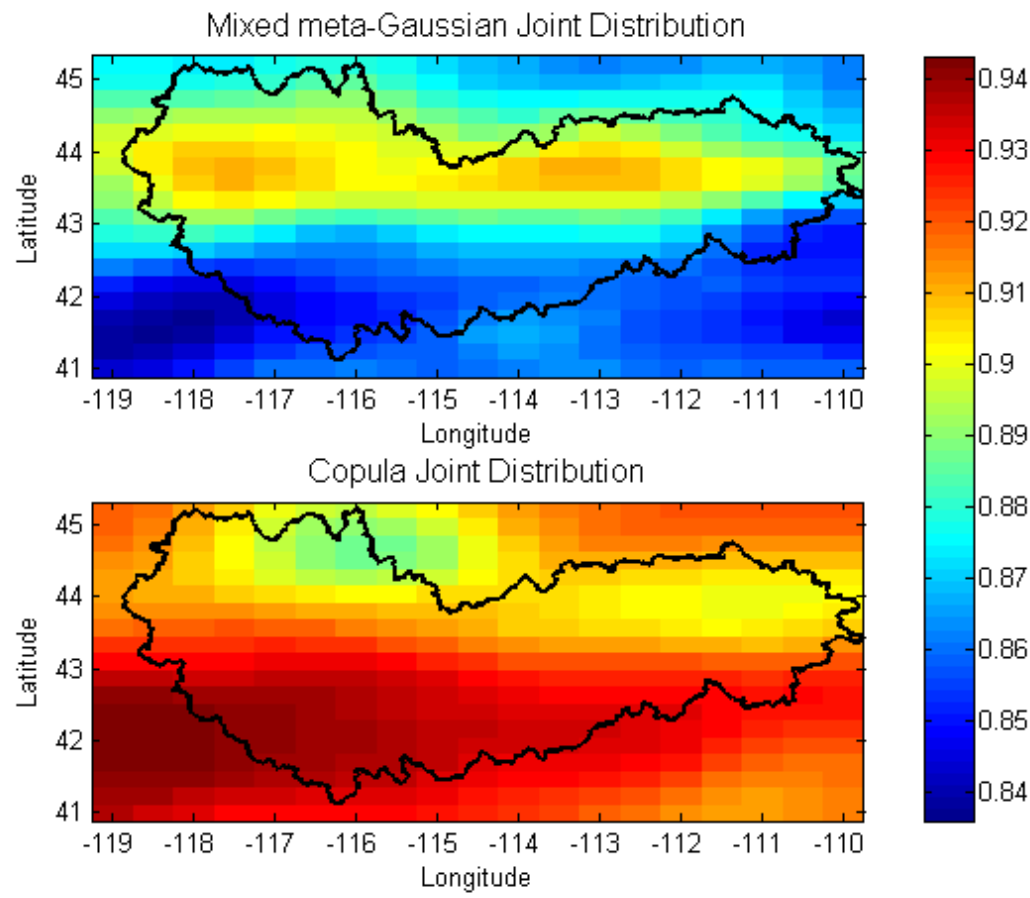

Figure 34 . Calculated KGE over Oxbow River Basin for two diverse generated mean precipitation ensemble forecast during calibration period. Meta-Gaussian approach (top) and Copula approach (bottom)

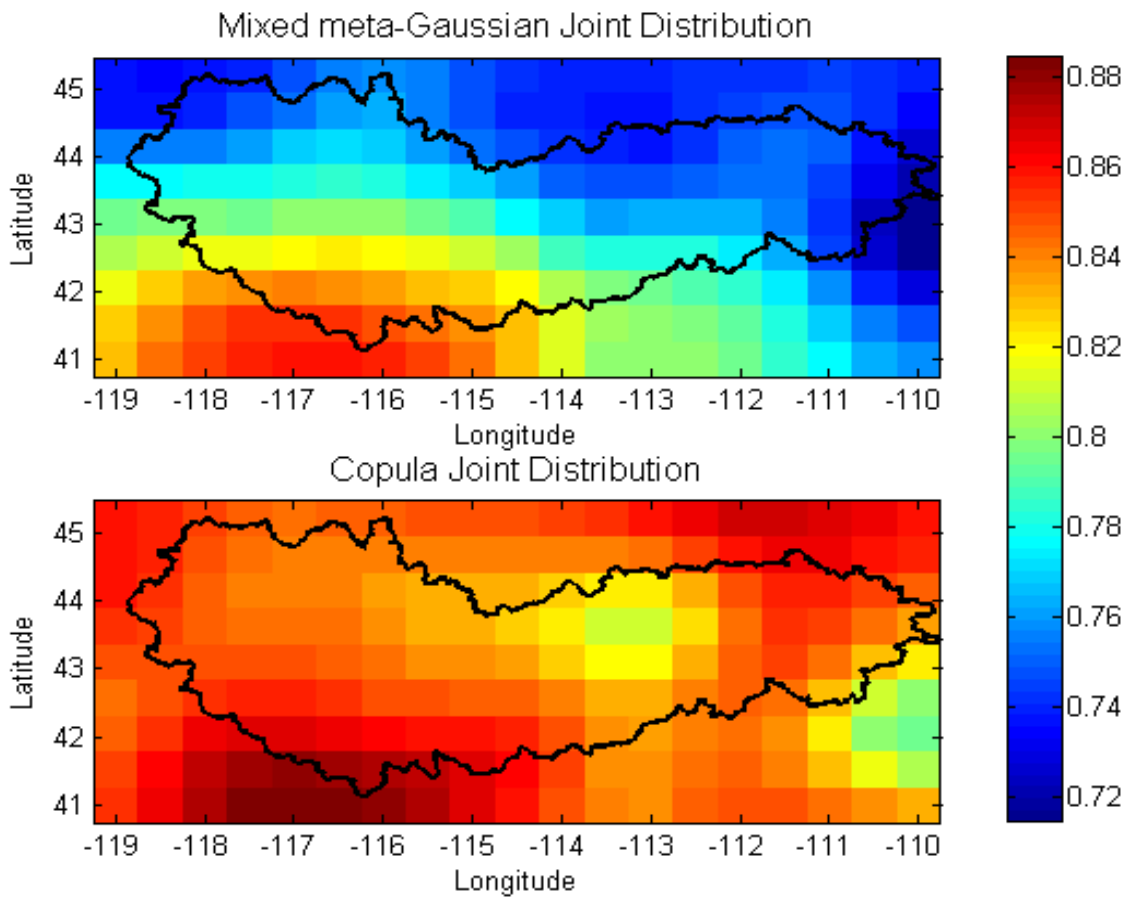

Figure 35 .Calculated KGE over Oxbow River Basin for two diverse generated mean precipitation ensemble forecast during validation period. Meta-Gaussian approach (top) and Copula approach (bottom) 


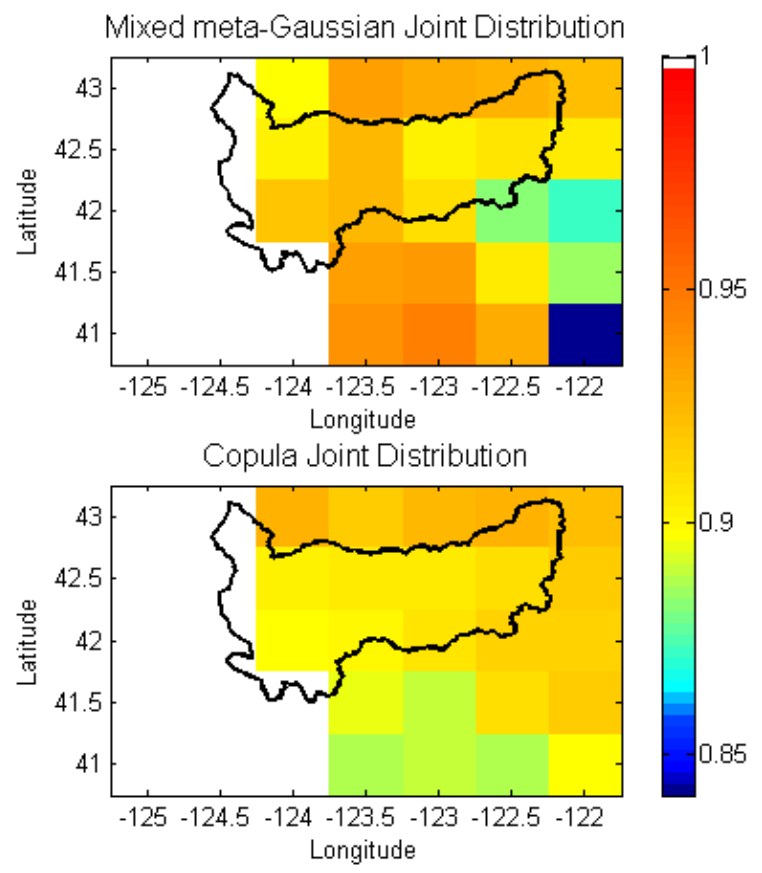

Figure 36 .Calculated KGE over Rouge River Basin for two diverse generated mean precipitation ensemble forecast during calibration period. Meta-Gaussian approach (top) and Copula approach (bottom)

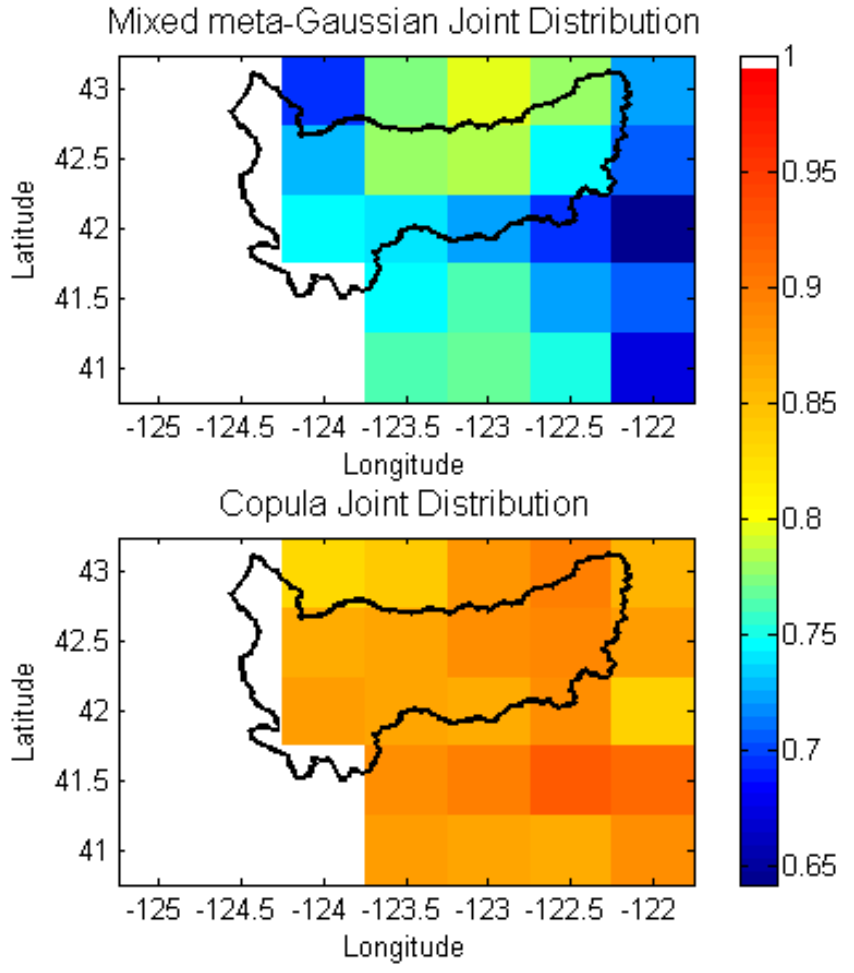

Figure 37 . Calculated KGE over Rouge River Basin for two diverse generated mean precipitation ensemble forecast during validation period. Meta-Gaussian approach (top) and Copula approach (bottom) 


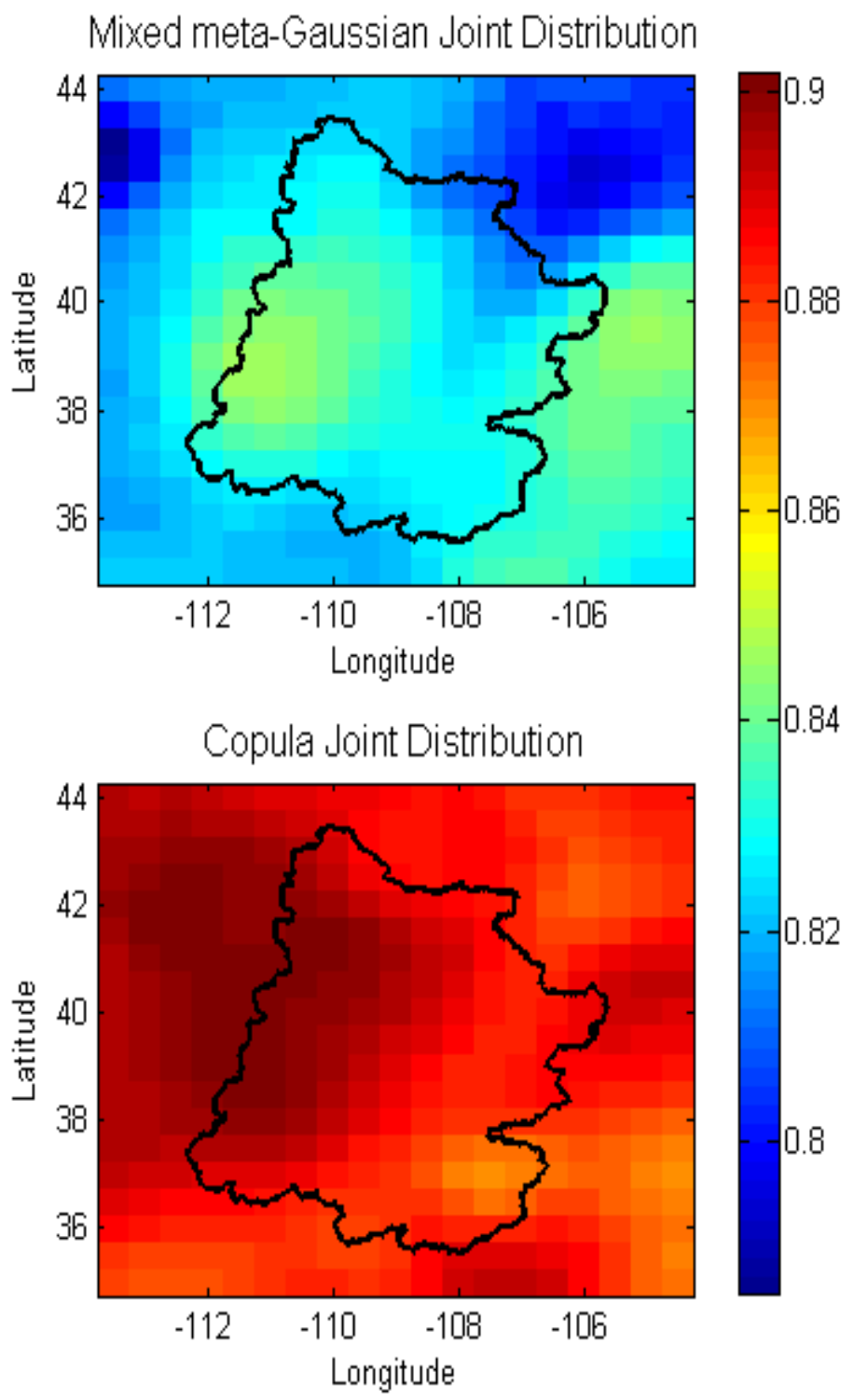

Figure 38 .Calculated NSE over Oxbow River Basin for two diverse generated mean precipitation ensemble forecast during calibration period. Meta-Gaussian approach (top) and Copula approach (bottom)

NSE measure also shows the same behavior as KGE for UCRB and Oxbow, in addition copula is dominant in the calibration period for Rouge River basin as well. Refer to figures 38 through 43 for details in improvement over spatial scale. 


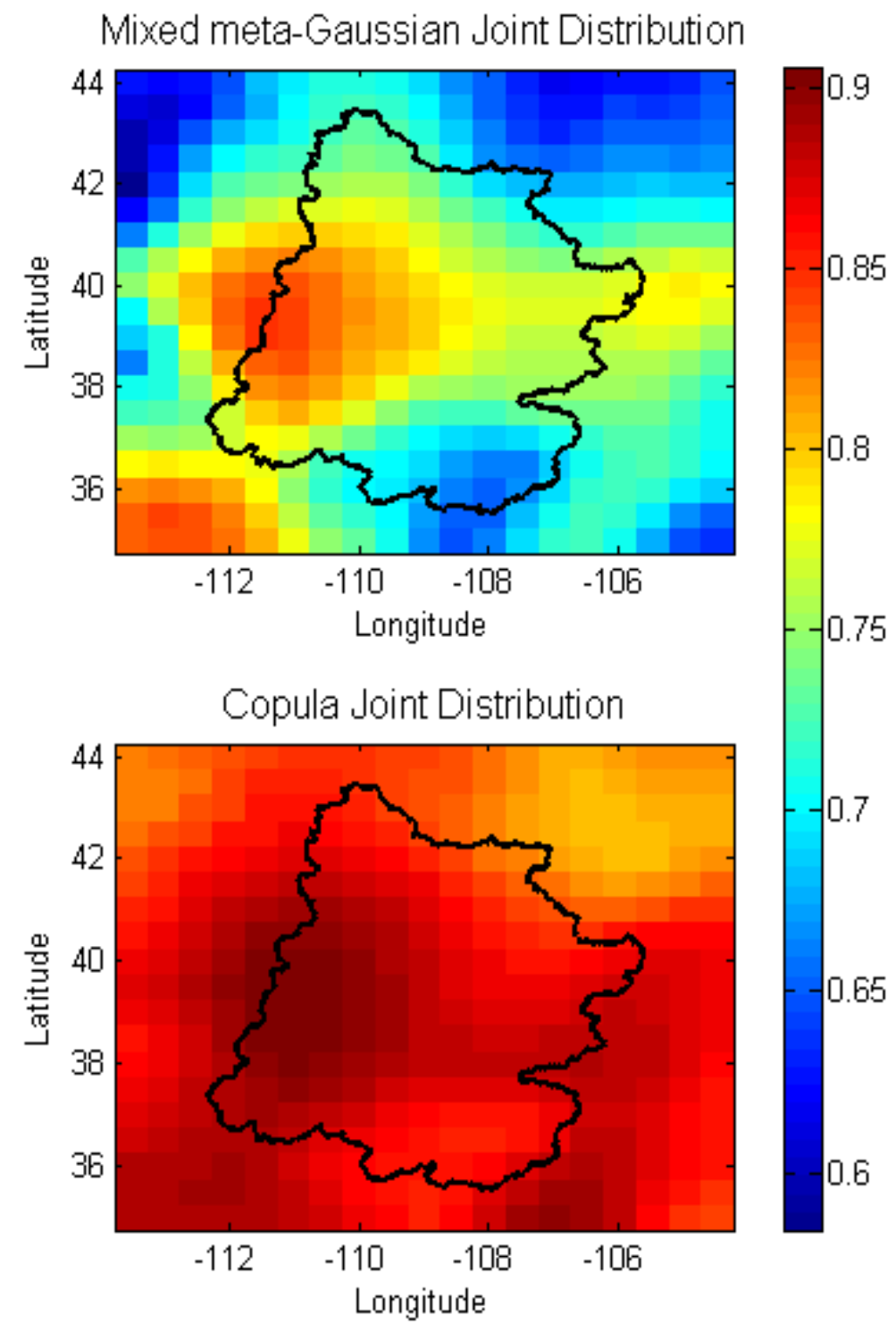

Figure 39 . Calculated NSE over Upper Colorado River Basin for two diverse generated mean precipitation ensemble forecast during validation period. Meta-Gaussian approach (top) and Copula approach (bottom) 


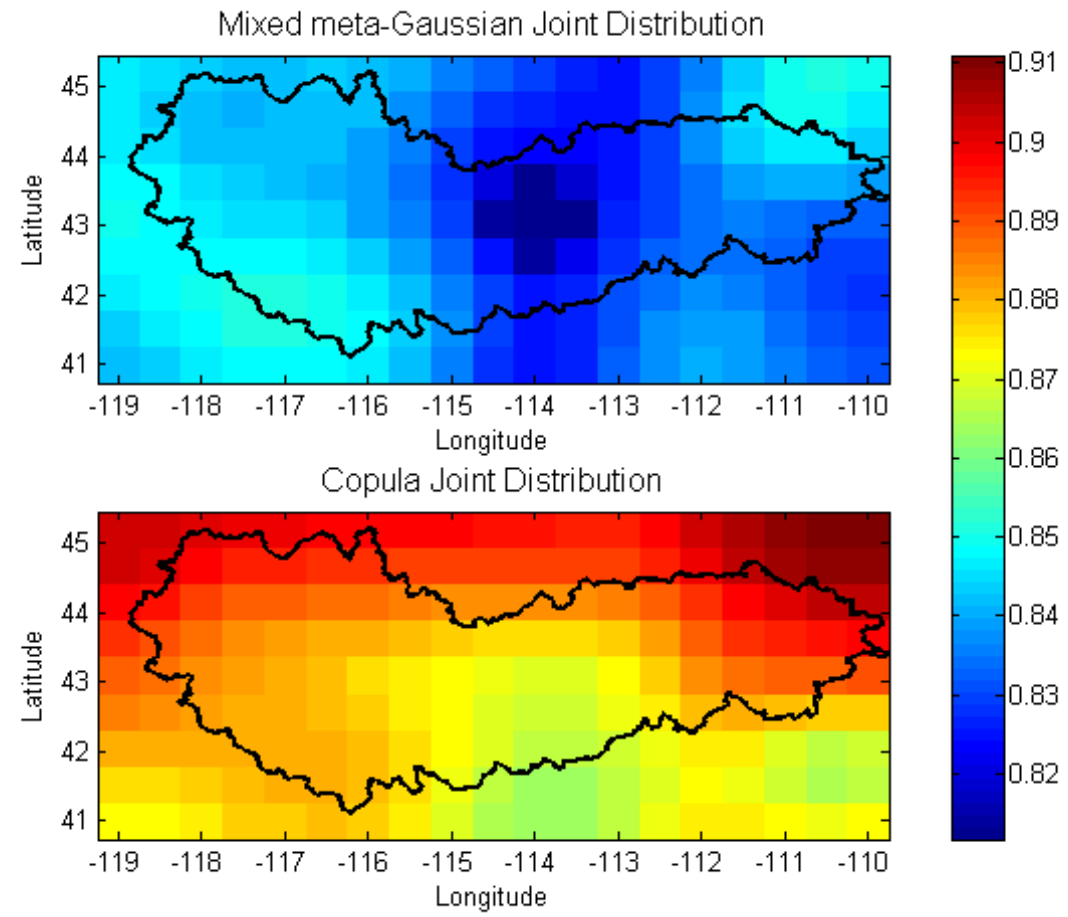

Figure 40 . Calculated NSE over Oxbow River Basin for two diverse generated mean precipitation ensemble forecast during calibration period. Meta-Gaussian approach (top) and Copula approach (bottom)

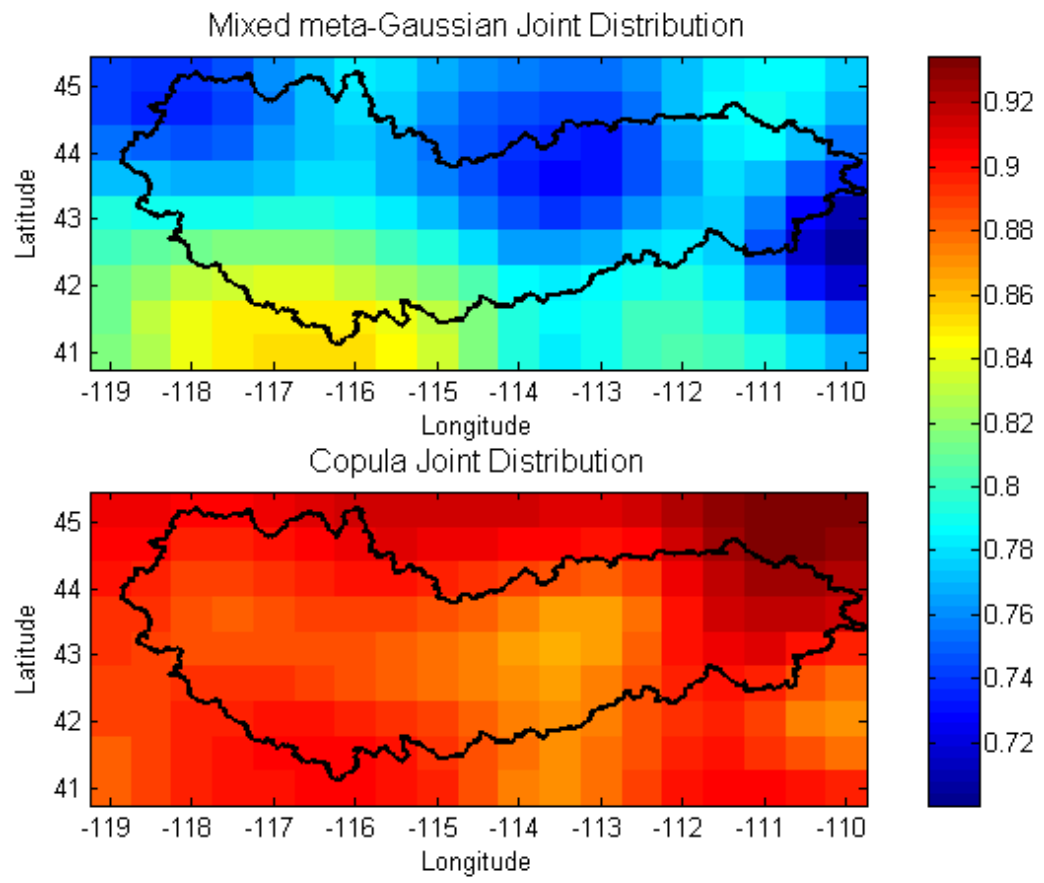

Figure 41 . Calculated NSE over Oxbow River Basin for two diverse generated mean precipitation ensemble forecast during validation period. Meta-Gaussian approach (top) and Copula approach (bottom) 


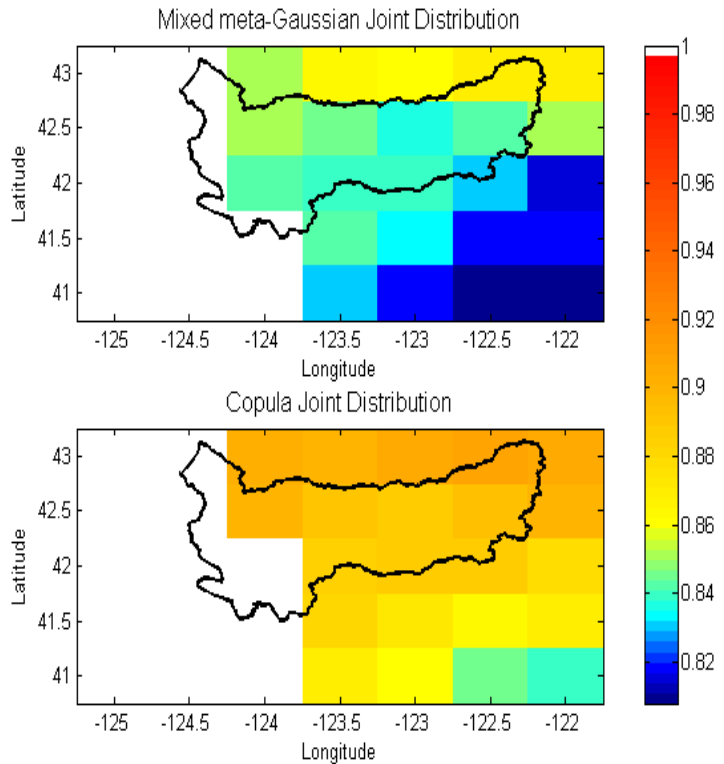

Figure 42. Calculated NSE over Rouge River Basin for two diverse generated mean precipitation ensemble forecast during calibration period. Meta-Gaussian approach (top) and Copula approach (bottom)

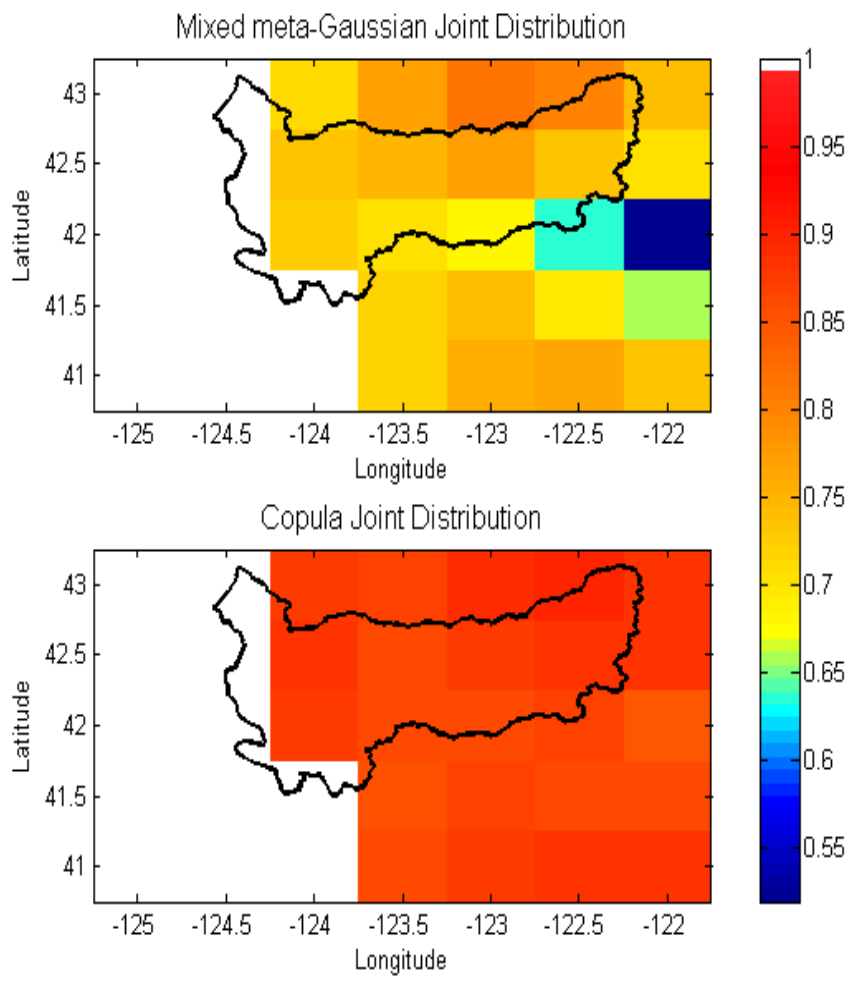

Figure 43 . Calculated NSE over Rouge River Basin for two diverse generated mean precipitation ensemble forecast during validation period. Meta-Gaussian approach (top) and Copula approach (bottom) 


\subsubsection{Probabilistic Forecast Verification}

It is also vital (and advisable) to examine the quantitative and reliability aspect of the generated ensemble precipitation using probabilistic framework. In regard to same, CRPSS, NRR, and exceedance ratio are employed. Figures (44) through (58) represents the probabilistic examination of the generated forecast using two different EPP methods. Figures 44 through 49 depicts results of CRPSS in calibration and validation period respectively with figures 50 through 55 presenting the same for NRR measure. Whereas figure 56-58 provides results of exceedance ratio at $95 \%$ in entire period. Results depicts that the measures in two periods, calibration and verification, are beneficial to assess the power/suitability of the methodology for application to the future forecast. 


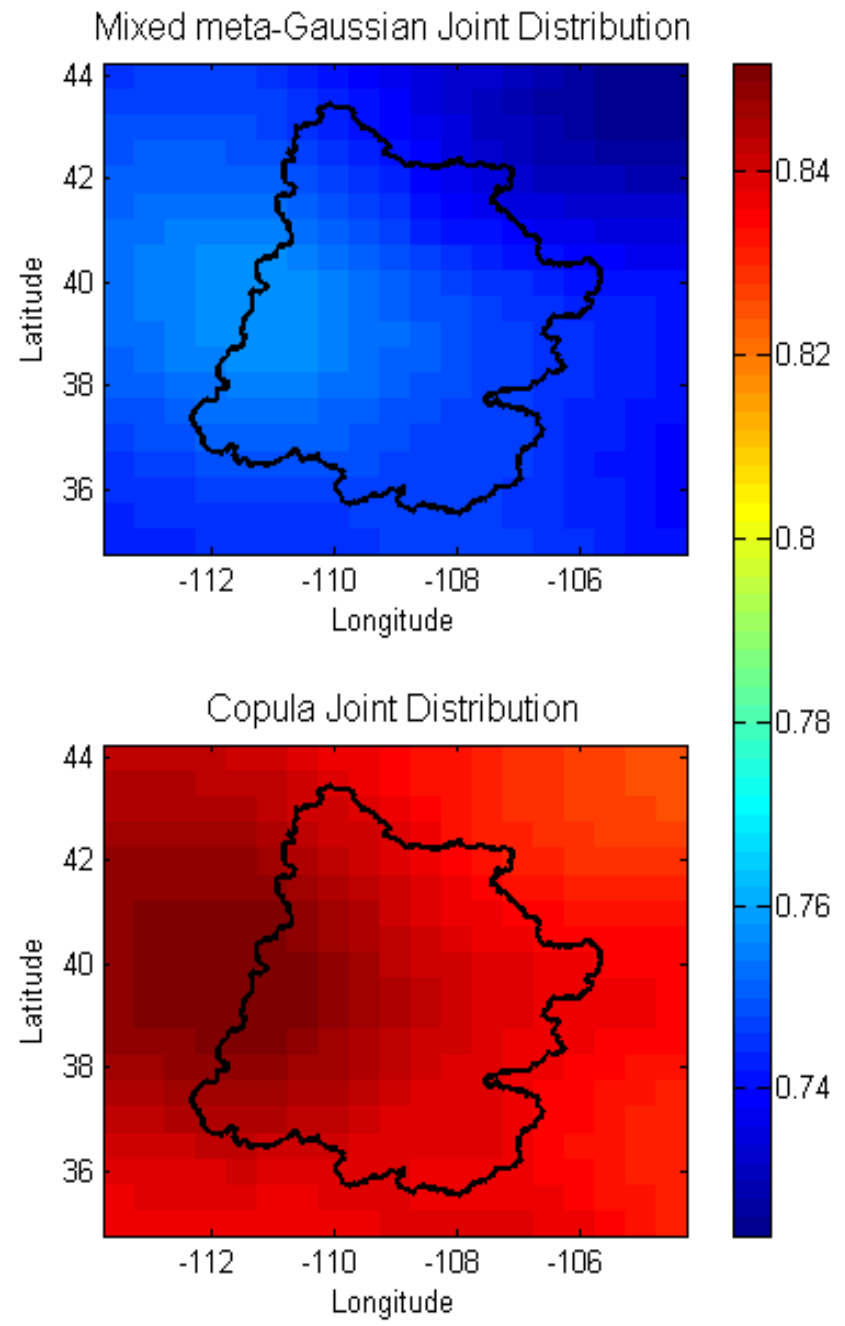

Figure 44 . Calculated CRPSS over Upper Colorado River Basin for two diverse generated precipitation ensemble forecast during calibration period. Meta-Gaussian approach (top) and Copula approach (bottom) 


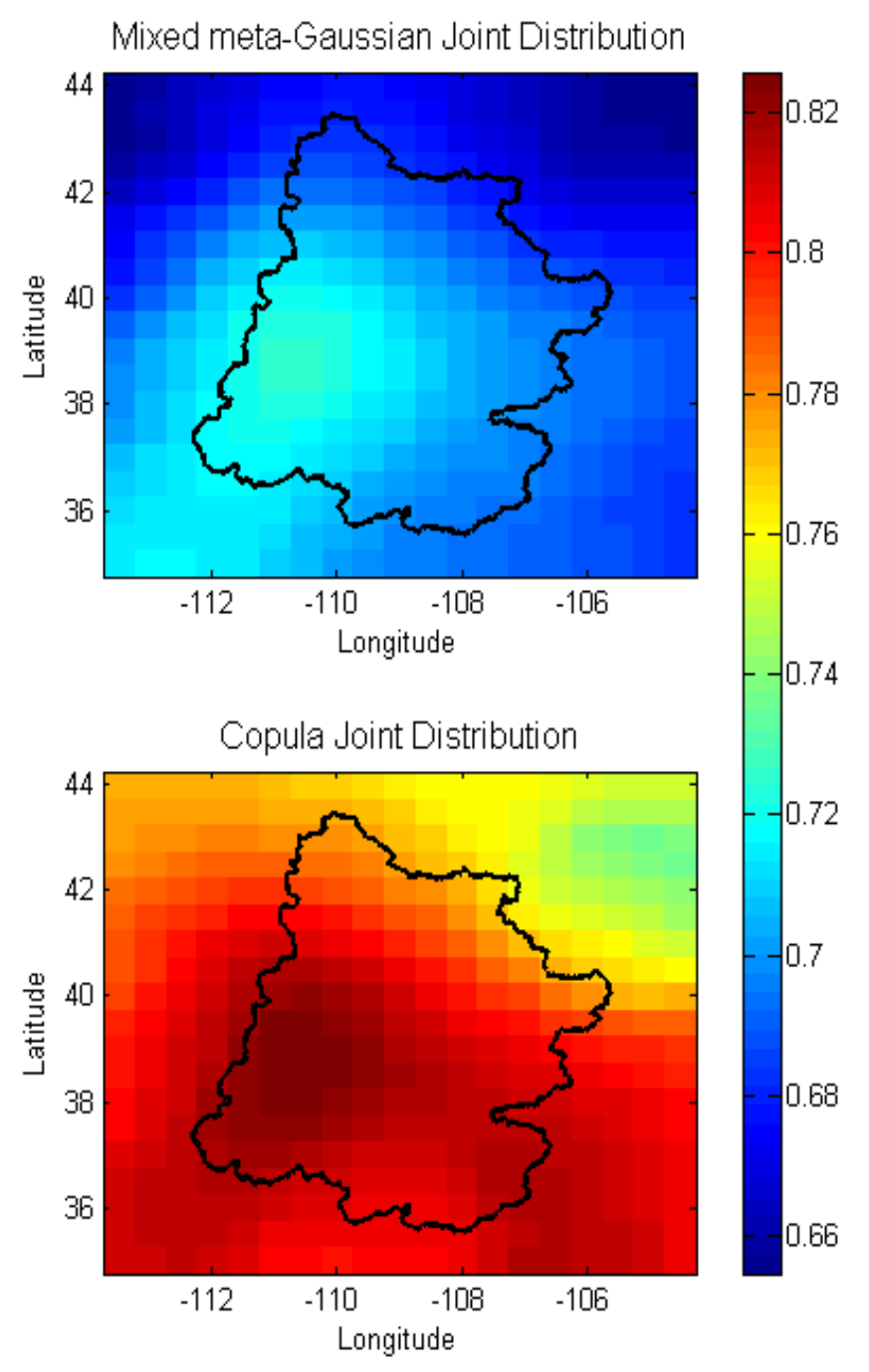

Figure 45 .Calculated CRPSS over Upper Colorado River Basin for two diverse generated precipitation ensemble forecast during validation period. Meta-Gaussian approach (top) and Copula approach (bottom)

Figure (44) to figure (49) represents the CRPSS measurements in the study areas.

For all the basins, both techniques are showing relatively high ranges in CRPSS. During the calibration period Copula based technique presents approximately $10 \%$ more improvement than MBG. Rouge basin shows higher ranges amongst the study basins, in 
other words the basins with more precipitation amount depicts more successful results after EPP.

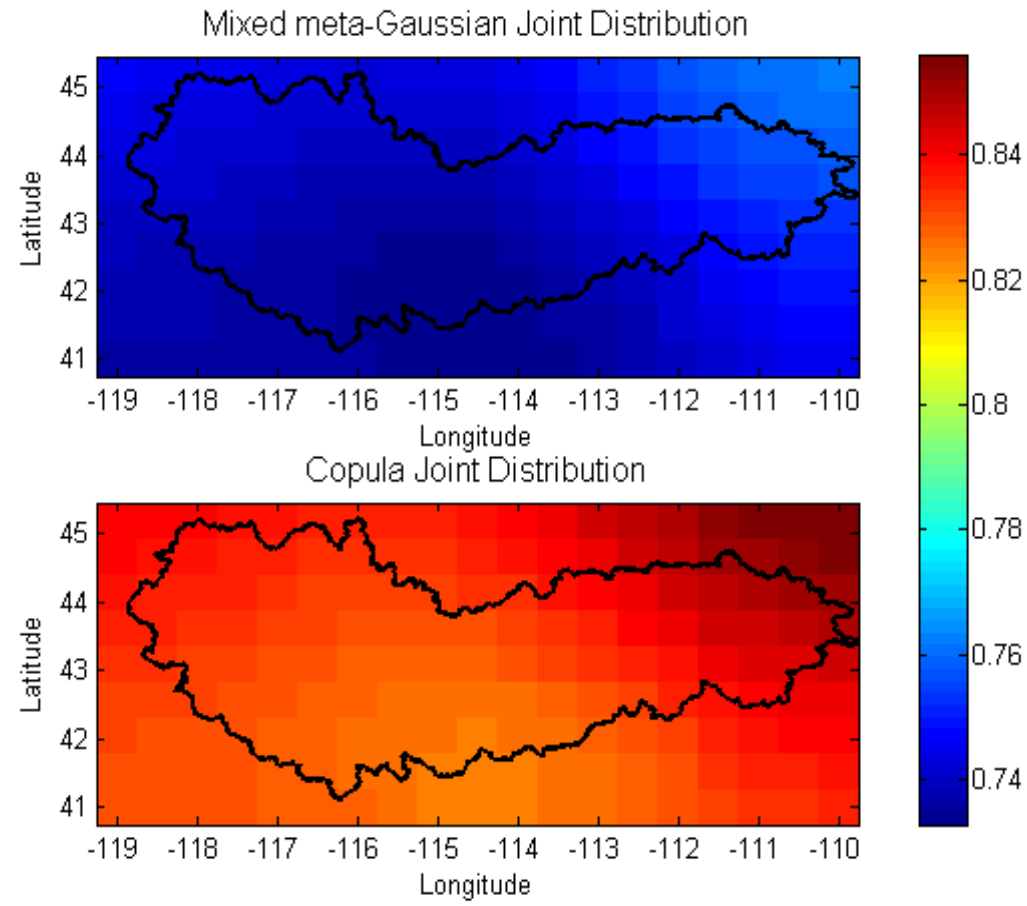

Figure 46 .Calculated CRPSS over Oxbow River Basin for two diverse generated precipitation ensemble forecast during calibration period. Meta-Gaussian approach (top) and Copula approach (bottom) 


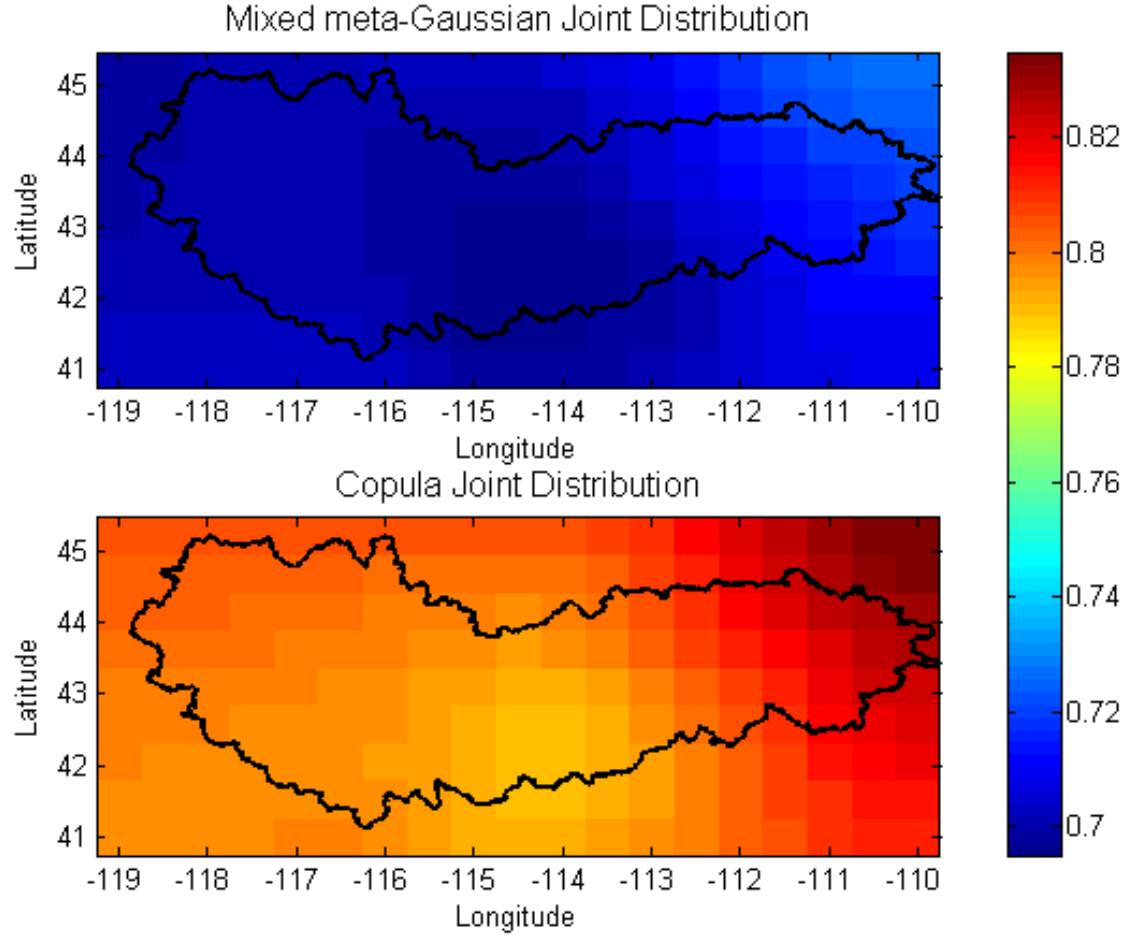

Figure 47 .Calculated CRPSS over Oxbow River Basin for two diverse generated precipitation ensemble forecast during validation period. Meta-Gaussian approach (top) and Copula approach (bottom) 


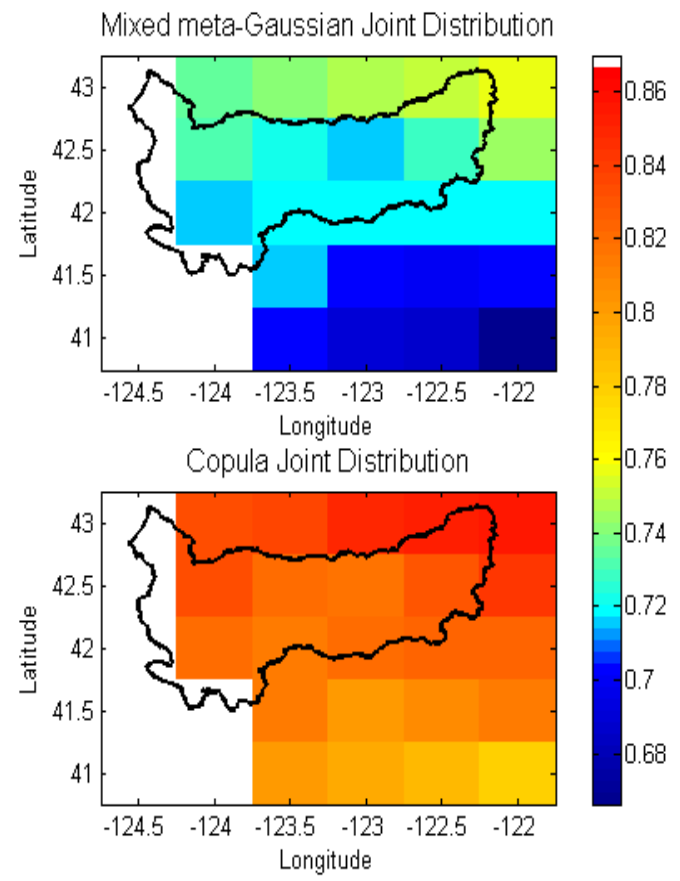

Figure 48.Calculated CRPSS over Rouge River Basin for two diverse generated precipitation ensemble forecast during calibration period. Meta-Gaussian approach (top) and Copula approach (bottom)

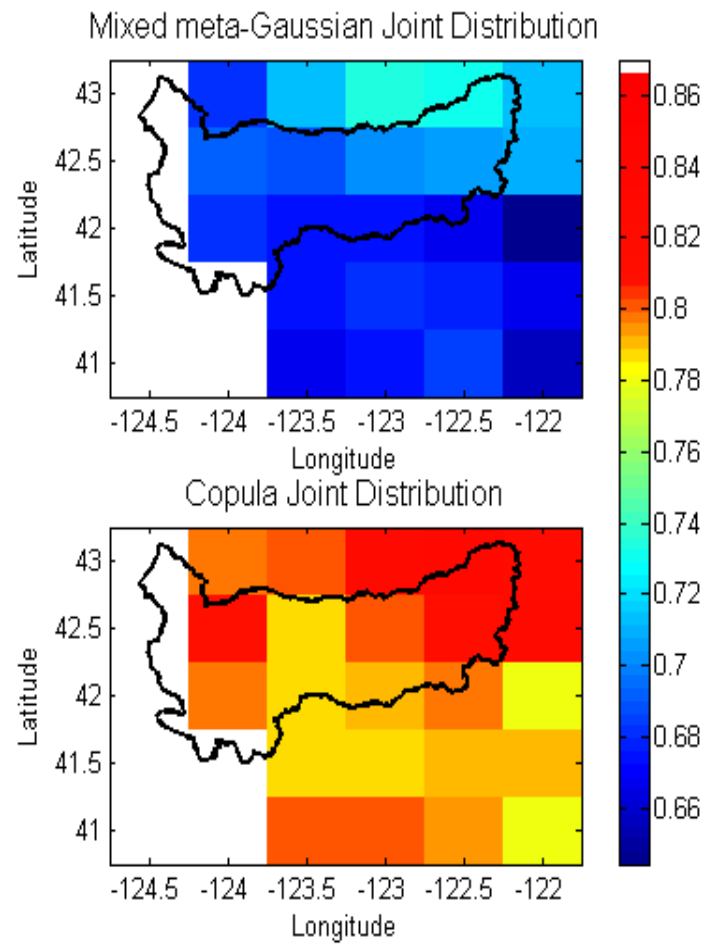

Figure 49 .Calculated CRPSS over Rouge River Basin for two diverse generated precipitation ensemble forecast during validation period. Meta-Gaussian approach (top) and Copula approach (bottom) 
During the validation period, CRPSS depicts lowered values as compared to the calibration period, however copula based technique is shrinking less than the metaGaussian method.

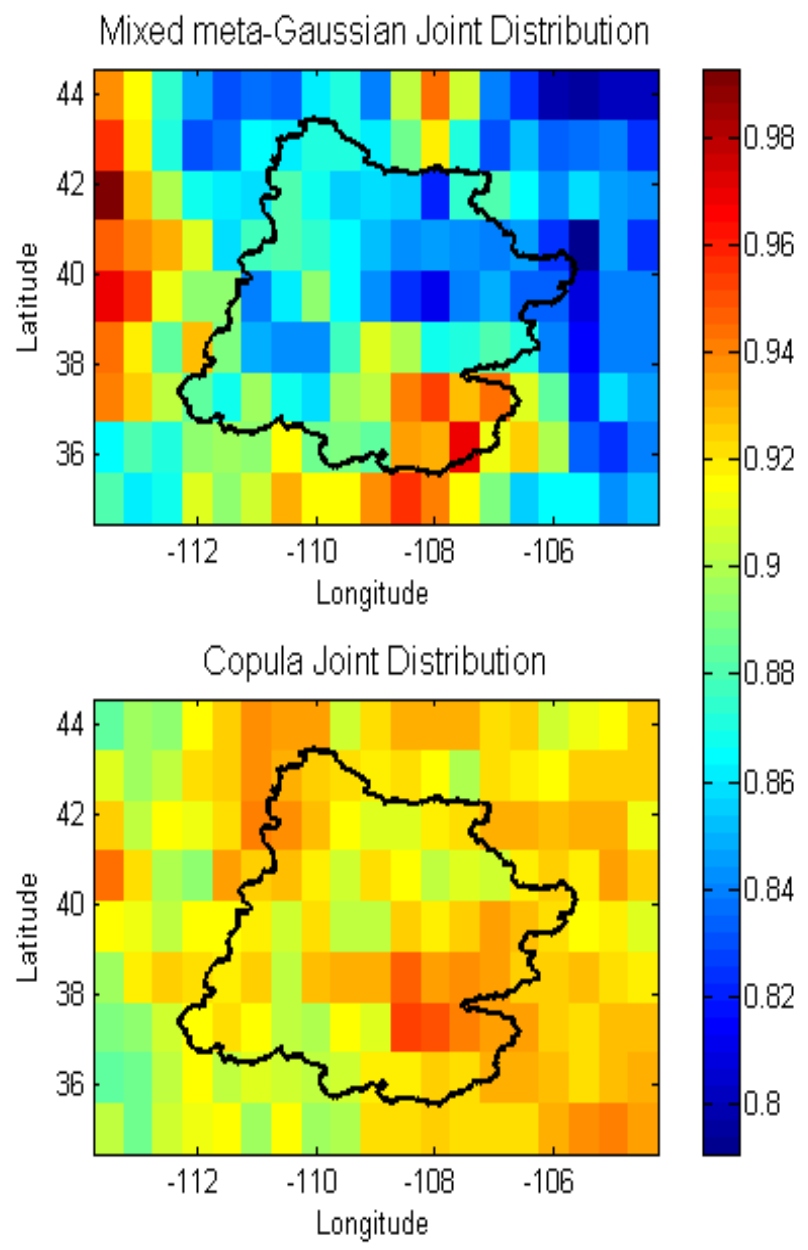

Figure 50 .Calculated NRR over Upper Colorado River Basin for two diverse generated precipitation ensemble forecast during calibration period. Meta-Gaussian approach (top) and Copula approach (bottom)

NRR is summarized in Figure (50) to figure (55) in the study area for calibration and validation periods. Upper Colorado Basin, during calibration, presents ensemble forecasts with higher spread, which in-turn represents the uncertainty in ensemble 
precipitation. Copula based technique has NRR ranges closer to the desirable ensemble $(\mathrm{NRR}=1)$ than meta- Gaussian procedure. During the validation, copula technique is preserving its behavior as in calibration period; on the other hand, for meta-Gaussian the forecasts shows little spread in some of the cells, which is more precise forecast with a little overconfidence.

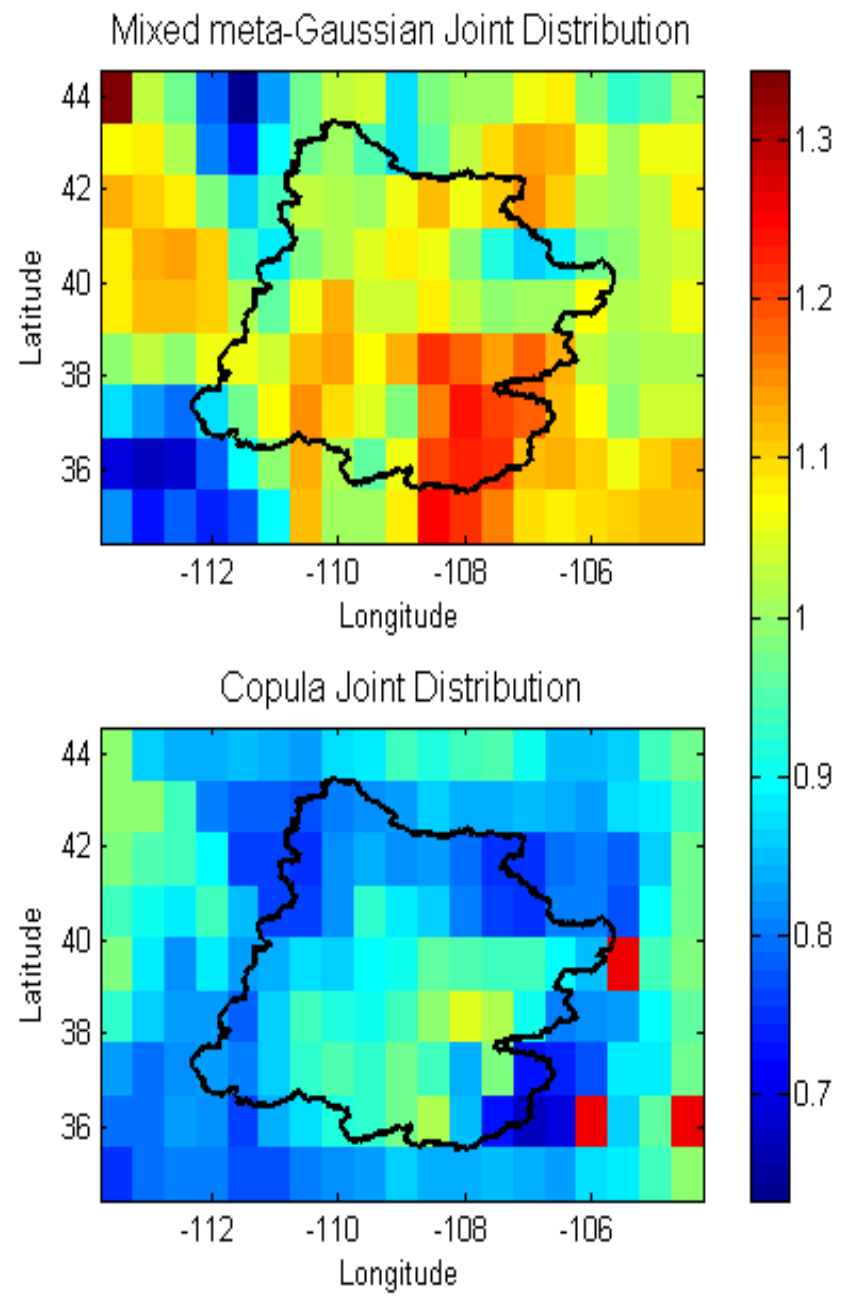

Figure 51 .Calculated NRR over Upper Colorado River Basin for two diverse generated precipitation ensemble forecast during validation period. Meta-Gaussian approach (top) and Copula approach (bottom) 
For Oxbow (figure 52 and 53), copula based forecast is showing almost the same trend for calibration as Upper Colorado. In meta-Gaussian forecast most of the cells are following the $\mathrm{NRR}<1$, however at some cells it is experiencing $\mathrm{NRR}>1$. However during the validation period, majority of the cells in Copula based forecast have NRR $<1$ whereas the meta-Gaussian does not show a consistent trend between the calibration and forecast.

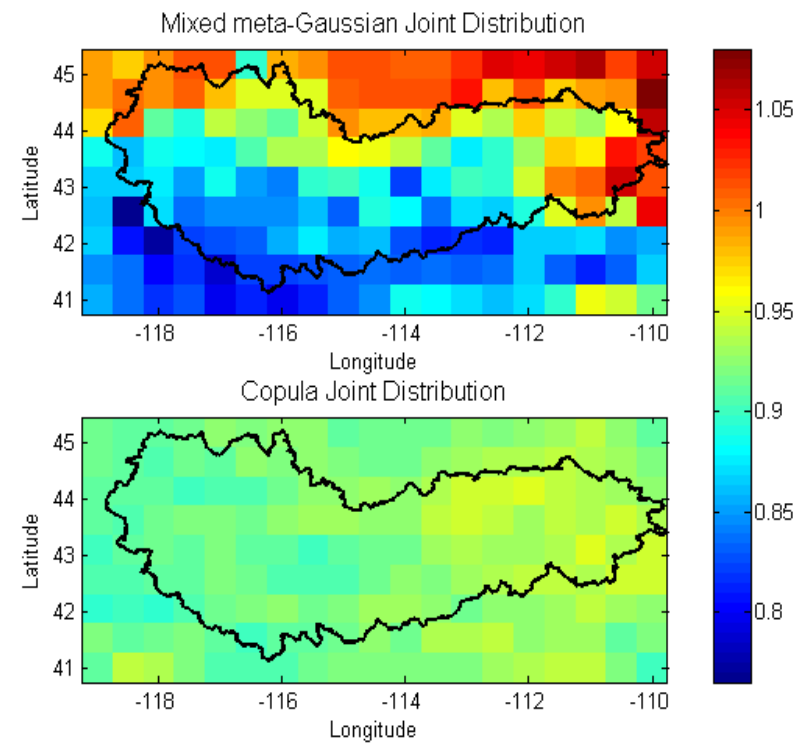

Figure 52.Calculated NRR over Oxbow River Basin for two diverse generated precipitation ensemble forecast during calibration period. Meta-Gaussian approach (top) and Copula approach (bottom) 

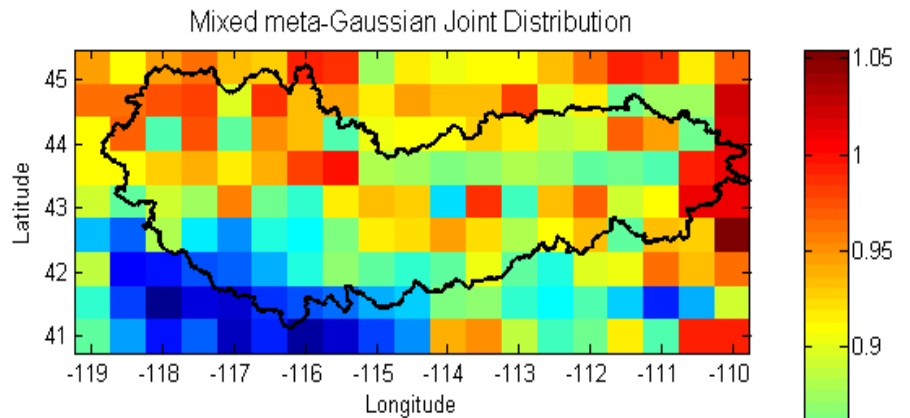

Copula Joint Distribution

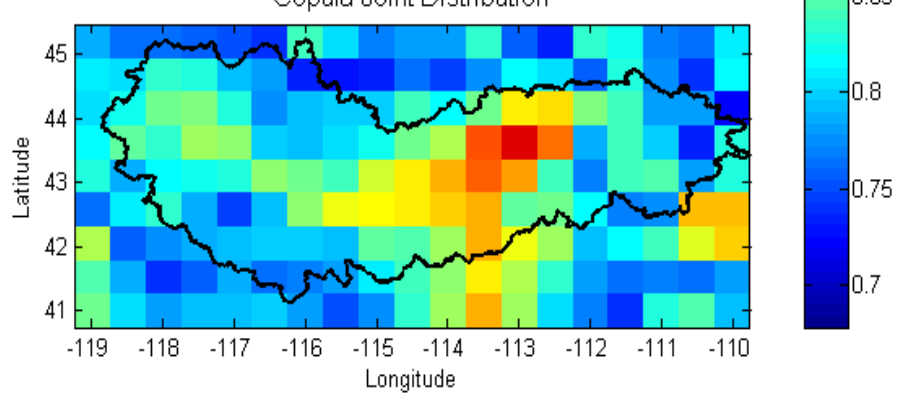

Figure 53 .Calculated NRR over Oxbow River Basin for two diverse generated precipitation ensemble forecast during validation period. Meta-Gaussian approach (top) and Copula approach (bottom)
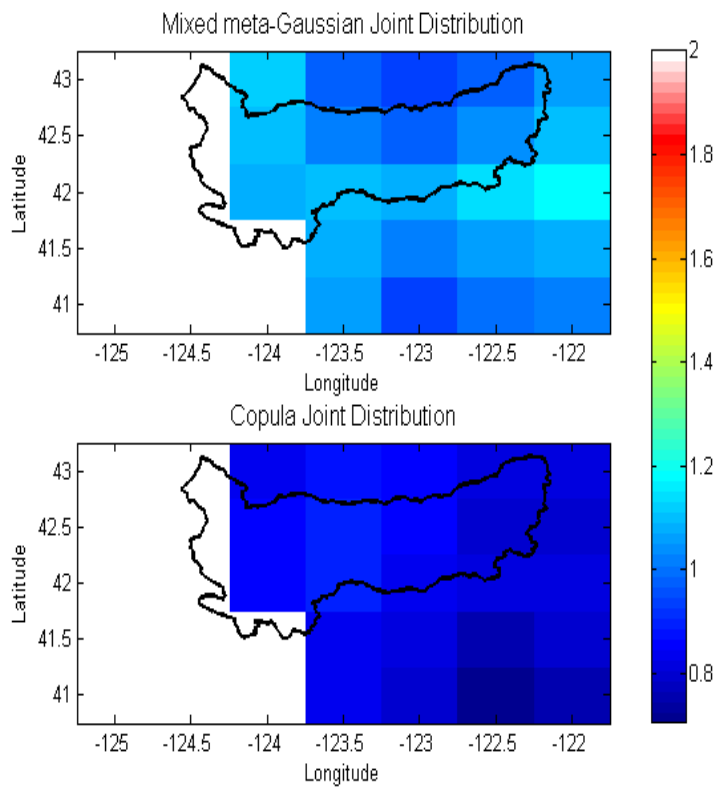

Figure 54 . Calculated NRR over Rouge River Basin for two diverse generated precipitation ensemble forecast during calibration period. Meta-Gaussian approach (top) and Copula approach (bottom) 


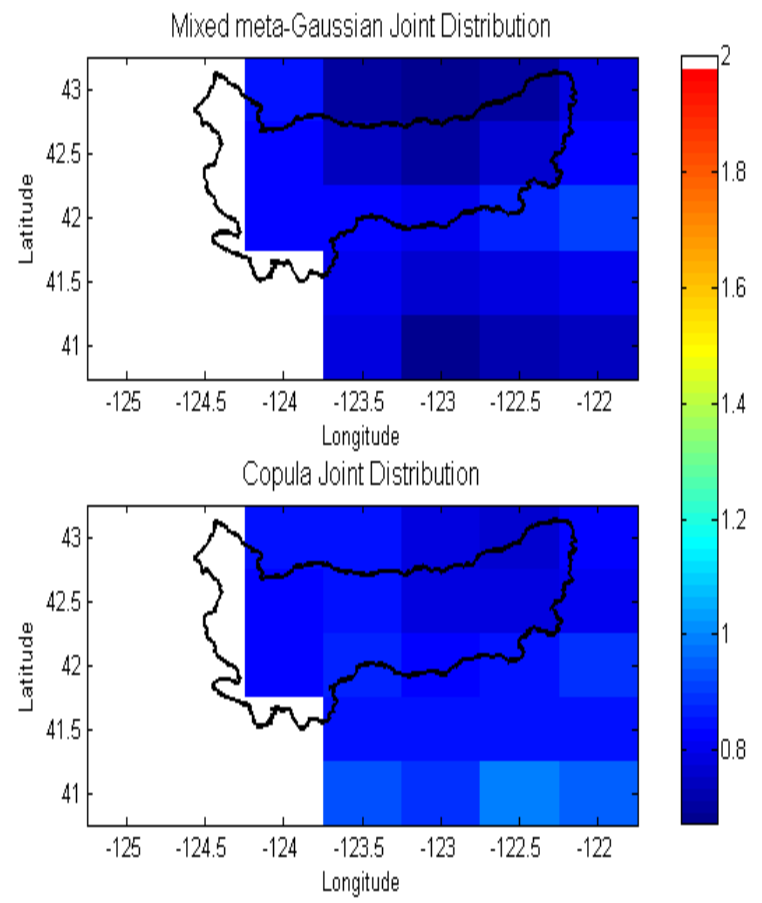

Figure 55.Calculated NRR over Rouge River Basin for two diverse generated precipitation ensemble forecast during validation period. Meta-Gaussian approach (top) and Copula approach (bottom)

In Rouge basin, meta-Gaussian is depicting different transition between the calibration and validation. During calibration it is presenting NRR $>1$ which during validation it is transferring to $\mathrm{NRR}<1$. The copula based forecast is following the same behavior as the other two basins.

Figure (56) to (58) represents the results of exceedance ratio at $95 \%$ quantile of the data. Due to short length of the observation and forecast during the validation period, ER is examined for the whole period of study (1979-2009) and not individually for the calibration and validation. 


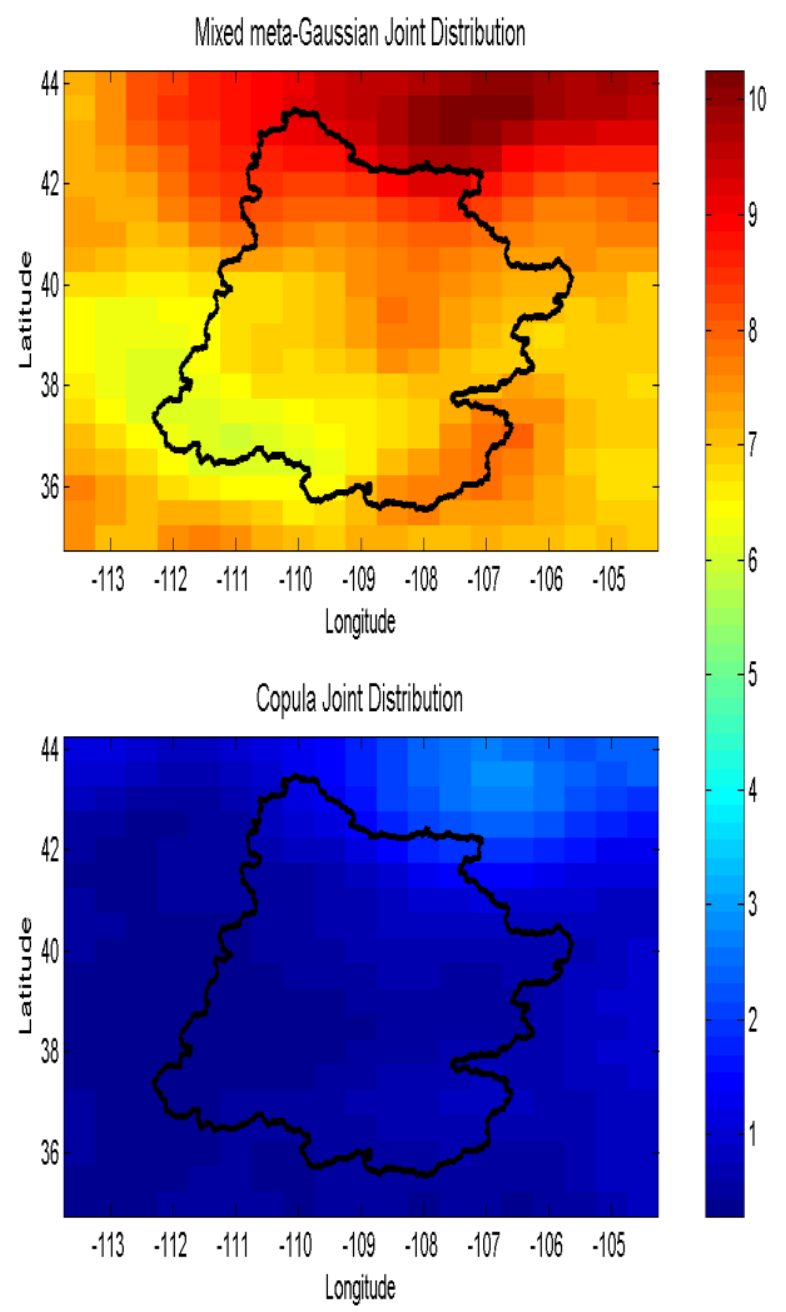

Figure 56.Calculated Exceedance Ratio for 95 percentile of the forecast data that the observation falls into over Upper Colorado River Basin for two diverse generated precipitation ensemble forecast during study period. Meta-Gaussian approach (top) and Copula approach (bottom)

Copula based EPP result for ER is almost less than $4 \%$ for the study area in three basin. This means that approximately all the observation are located between the $95 \%$ quantile of generated ensemble forecast. Despite the significant differences between two methods, MBG also shows promising results for exceedance ratio for all the basins. 


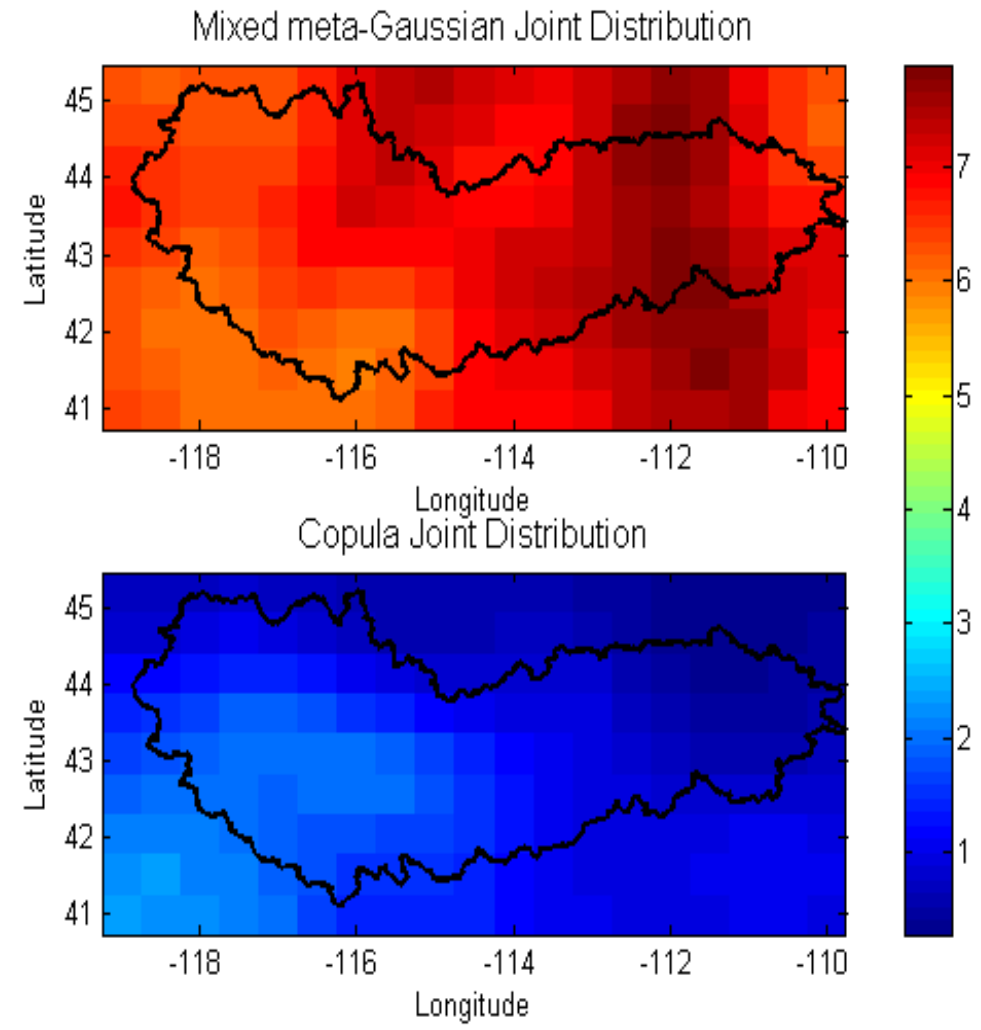

Figure 57.Calculated Exceedance Ratio for 95 percentile of the forecast data that the observation falls into, over Oxbow River Basin for two diverse generated precipitation ensemble forecast during study period. Meta-Gaussian approach (top) and Copula approach (bottom) 

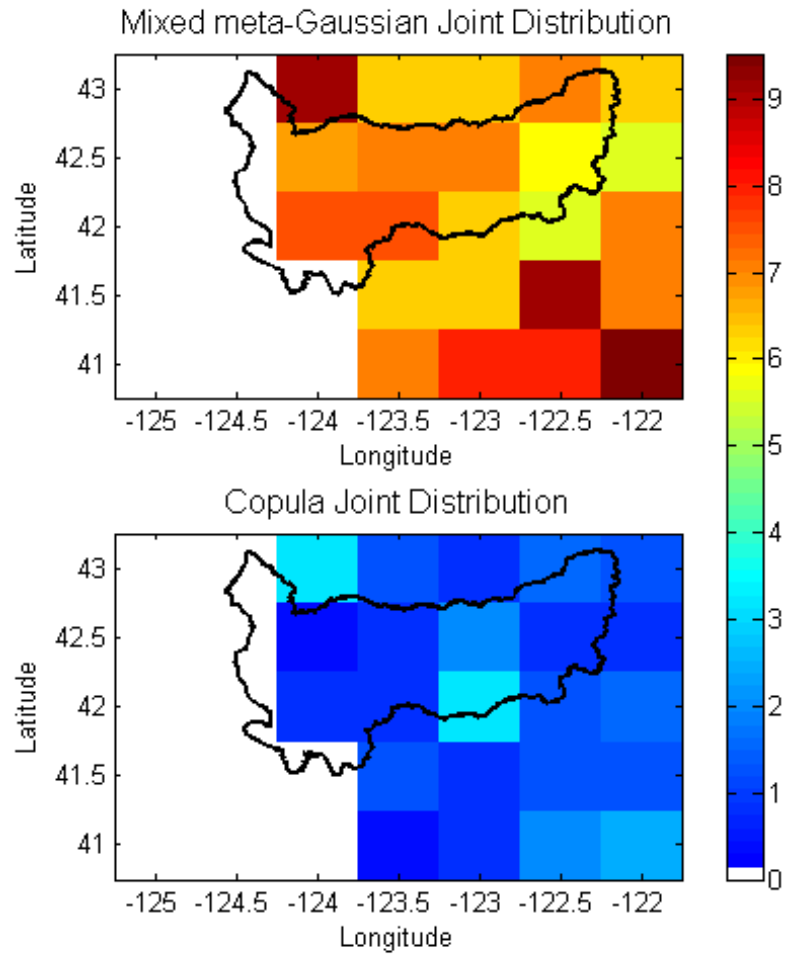

Figure 58. Calculated Exceedance Ratio for 95 percentile of the forecast data that the observation falls into, over Rouge River Basin for two diverse generated precipitation ensemble forecast during study period. Meta-Gaussian approach (top) and Copula approach (bottom) 


\section{Discussion and Conclusion}

The focus of this study was to assess/examine the reliability of the proposed methodology, i.e. utilization of copula functions to generate ensemble precipitation forecast (EPP), based on the relation between the single-value precipitation forecast and historical observation. Further the goal included comparison of copula based EPP with MBG, a well-known procedure for the task, in 2 study basins.

The examination was performed using deterministic and probabilistic forecast verification methods due to various aspects highlighted by them. Overall, both techniques were successful in building reliable ensemble precipitation forecasts both in calibration period and verification segment as compared to raw forecasts. This further supports the idea of emphasizing the need for techniques (bias correction) in generating reliable and certain forecast products from climatological observations before using them in hydrological studies.

In the assessment of the proposed approach, the deterministic framework, pointed towards significantly smaller errors in the mean ensemble forecasts using copula based generated ensemble than using meta-Gaussian. In accuracy examination of the study, NSE depicted more accurate mean ensemble to match observation. KGE was employed to study the correlation between the generated and observation forecast, which depicted that the correlation between copula based generated forecast is consequentially higher than metaGaussian ensemble forecast. Overall, copula based ensemble forecast demonstrates promising improvement throughout the deterministic framework. 
Based on increasing reliability of the probabilistic forecast verification methods, it was essential to examine the generated forecast through the framework. Same as the deterministic results, both techniques employed in the study showed promising results in pre-processing of precipitation forecast than the raw forecast. CRPSS was used to highlight differences in reliability and uncertainty of ensemble forecast with that of raw forecast. Results of CRPSS indicates that copula based forecasts are generating precipitation predictions which are more reliable, accurate, and with higher resolution compared to the meta-Gaussian one.

NRR was employed to study the ensemble spread, wherein results indicated that the copula based forecasts have almost $\mathrm{NRR}<1$, which represents the uncertainty in the forecast. On the other hand, meta-Gaussian forecast does not follow a consistent trend, with precise ensemble spread in certain spatial cells and some indicating otherwise (i.e. wide spread). It is also important to point out that Copula based forecasts are retaining the spread throughout the verification period, which is not the case for the meta-Gaussian ensemble forecast.

Exceedance Ratio was further used to assess the uncertainty in the generated ensemble. It pointed towards success in description of observation, through the study period, using both methods. However, copula based forecasts were successful predicting forecast that are much more reliable at $95 \%$ than $\mathrm{MBG}$ resulting in indicating helpfulness of same in extreme cases, if they appear in the data.

To conclude, copula was observed to be more precise in building the ensemble precipitation forecast both in the deterministic and probabilistic frameworks. The most 
significant aspect of copula-based approach was consistency in the generated results. Accordingly, it can be demonstrated from the results that the technique is almost independent of spatial and temporal change in the data. Similarly, in the verification period, comparison to the calibration one emphasizes on the robustness of the technique, which meta-Gaussian procedure fail to produce.

Finally, I would like to bring to your attention that by adapting copula functions into Ensemble Pre-Processing, we can successfully overcome the requirement of normal distribution for the observation and forecast. This would result in eliminating the transformation step used in current techniques. One of the most important advantages of employing copula function is its capability of describing the joint distribution between the forecast and observation with any level of dependency. Moreover, copula function has shown to be helpful in ability to handle the extreme values in the observation and generated ensemble forecast. 


\section{Future Work}

1. The same study could be applied on the ensembles by the climate forecasters and compare the same ensemble forecast with the existing one using copula.

2. This technique is applicable for pre-processing temperature as well. Therefore, it would be beneficial to see the effect of the procedure on temperature.

3. This study has been done on the monthly precipitation. Due to the increase in demands for short-range forecast, it is vital to conduct the study on daily or sub-daily forecasts.

4. The main purpose of this evaluation was preparing the forecast precipitation for hydrologic applications. In the future, the generated results should be adapted to the hydrologic model as input precipitation to examine the effect of EPP on hydrologic forecast. 


\section{References}

Aho, K., D. Derryberry, and T. Peterson, 2014, Model selection for ecologists: the worldviews of AIC and BIC: Ecology, v. 95, p. 631-636.

Anderson, J. L., 2001, An ensemble adjustment Kalman filter for data assimilation: Monthly weather review, v. 129, p. 2884-2903.

Anderson, T. W., 1962, On the distribution of the two-sample Cramer-von Mises criterion: The Annals of Mathematical Statistics, p. 1148-1159.

Borga, M., 2002, Accuracy of radar rainfall estimates for streamflow simulation: Journal of Hydrology, v. 267, p. 26-39.

Brown, J. D., and D. J. Seo, 2013, Evaluation of a nonparametric post-processor for bias correction and uncertainty estimation of hydrologic predictions: Hydrological Processes, v. 27, p. 83-105.

Bárdossy, A., 2006, Copula-based geostatistical models for groundwater quality parameters: Water Resources Research, v. 42.

Cherubini, U., E. Luciano, and W. Vecchiato, 2004, Copula methods in finance, John Wiley \& Sons.

Clark, M., S. Gangopadhyay, L. Hay, B. Rajagopalan, and R. Wilby, 2004, The Schaake shuffle: A method for reconstructing space-time variability in forecasted precipitation and temperature fields: Journal of Hydrometeorology, v. 5, p. 243262.

Clark, M. P., and L. E. Hay, 2004, Use of medium-range numerical weather prediction model output to produce forecasts of streamflow: Journal of Hydrometeorology, $\mathrm{v}$. 5, p. 15-32.

DeChant, C. M., and H. Moradkhani, 2011, Improving the characterization of initial condition for ensemble streamflow prediction using data assimilation: Hydrology and Earth System Sciences, v. 15, p. 3399-3410.

DeChant, C. M., and H. Moradkhani, 2014, Toward a reliable prediction of seasonal forecast uncertainty: Addressing model and initial condition uncertainty with ensemble data assimilation and Sequential Bayesian Combination: Journal of Hydrology, v. 519, Part D, p. 2967-2977. 
DeChant, C.M., and H. Moradkhani (2015), On the Assessment of Reliability in Probabilistic Hydro-meteorological Event Forecasting, Water Resources Research, DOI: 10.1002/2014WR016617.

Demargne, J., J. Brown, Y. Liu, D. J. Seo, L. Wu, Z. Toth, and Y. Zhu, 2010, Diagnostic verification of hydrometeorological and hydrologic ensembles: Atmospheric Science Letters, v. 11, p. 114-122.

Favre, A. C., S. El Adlouni, L. Perreault, N. Thiémonge, and B. Bobée, 2004, Multivariate hydrological frequency analysis using copulas: Water resources research, v. 40.

Genest, C., B. Rémillard, and D. Beaudoin, 2009, Goodness-of-fit tests for copulas: A review and a power study: Insurance: Mathematics and economics, v. 44, p. 199213.

Gupta, H. V., H. Kling, K. K. Yilmaz, and G. F. Martinez, 2009, Decomposition of the mean squared error and NSE performance criteria: Implications for improving hydrological modelling: Journal of Hydrology, v. 377, p. 80-91.

Hamill, T. M., and J. S. Whitaker, 2006, Probabilistic quantitative precipitation forecasts based on reforecast analogs: Theory and application: Monthly Weather Review, v. 134 , p. 3209-3229.

Hamill, T. M., J. S. Whitaker, and S. L. Mullen, 2006, Reforecasts: An Important Dataset for Improving Weather Predictions: Bulletin of the American Meteorological Society, v. 87, p. 33-46.

Hersbach, H., 2000, Decomposition of the Continuous Ranked Probability Score for Ensemble Prediction Systems: Weather and Forecasting, v. 15, p. 559-570.

Joe, H., 1997, Multivariate models and multivariate dependence concepts, v. 73, CRC Press.

Kelly, K. S., and R. Krzysztofowicz, 1997, A bivariate meta-Gaussian density for use in hydrology: Stochastic Hydrology and Hydraulics, v. 11, p. 17-31.

Krzysztofowicz, R., and H. D. Herr, 2001, Hydrologic uncertainty processor for probabilistic river stage forecasting: precipitation-dependent model: Journal of Hydrology, v. 249, p. 46-68.

Li, H., J. Sheffield, and E. F. Wood, 2010, Bias correction of monthly precipitation and temperature fields from Intergovernmental Panel on Climate Change AR4 models using equidistant quantile matching: Journal of Geophysical Research: Atmospheres (1984-2012), v. 115. 
Liu, Y., Q. Duan, L. Zhao, A. Ye, Y. Tao, C. Miao, X. Mu, and J. C. Schaake, 2013, Evaluating the predictive skill of post-processed NCEP GFS ensemble precipitation forecasts in China's Huai river basin: Hydrological Processes, v. 27, p. 57-74.

Madadgar, S., and H. Moradkhani, 2014, Improved Bayesian multimodeling: Integration of copulas and Bayesian model averaging: Water Resources Research, 50, 95869603, DOI: 10.1002/2014WR015965.

Madadgar, S., H. Moradkhani, and D. Garen, 2014, Towards improved post-processing of hydrologic forecast ensembles: Hydrological Processes, v. 28, p. 104-122.

Madadgar, S., and H. Moradkhani, (2013), Drought Analysis under Climate Change using Copula, Journal of Hydrologic Engineering, 18 (7), doi:http://dx.doi.org/10.1061/(ASCE)HE.1943-5584.0000532.

Madadgar, S. and H. Moradkhani (2013), A Bayesian Framework for Probabilistic Drought Forecasting, Journal of Hydrometeorology, special issue of Advances in Drought Monitoring, 14, 1685-1705, DOI: 10.1175/JHM-D-13-010.1

Madadgar, S., and H. Moradkhani (2014), Spatio-temporal Drought Forecasting within Bayesian Networks, Journal of Hydrology, 512, 134-146, DOI: 10.1016/j.jhydrol.2014.02.039.

Moradkhani, H., C. M. DeChant, and S. Sorooshian, 2012, Evolution of ensemble data assimilation for uncertainty quantification using the particle filter-Markov chain Monte Carlo method: Water Resources Research, v. 48, W12520, doi:10.1029/2012WR012144.

Moradkhani, H., K. Hsu, Y. Hong, and S. Sorooshian, 2006, Investigating the impact of remotely sensed precipitation and hydrologic model uncertainties on the ensemble streamflow forecasting: Geophysical research letters, v. 33.

Moradkhani, H., K. L. Hsu, H. Gupta, and S. Sorooshian, 2005a, Uncertainty assessment of hydrologic model states and parameters: Sequential data assimilation using the particle filter: Water Resources Research, v. 41.

Moradkhani, H., and S. Sorooshian, 2008, General review of rainfall-runoff modeling: model calibration, data assimilation, and uncertainty analysis, Hydrological Modelling and the Water Cycle, Springer, p. 1-24.

Moradkhani, H., S. Sorooshian, H. V. Gupta, and P. R. Houser, 2005b, Dual stateparameter estimation of hydrological models using ensemble Kalman filter: Advances in Water Resources, v. 28, p. 135-147. 
Najafi, M. R., and H. Moradkhani, 2014, A hierarchical Bayesian approach for the analysis of climate change impact on runoff extremes: Hydrological Processes, v. 28, p. 6292-6308.

Nash, J. E., and J. V. Sutcliffe, 1970, River flow forecasting through conceptual models part I - A discussion of principles: Journal of Hydrology, v. 10, p. 282-290.

Nelsen, R. B., 1999, An introduction to copulas, Springer.

Park, Y. Y., R. Buizza, and M. Leutbecher, 2008, TIGGE: Preliminary results on comparing and combining ensembles: Quarterly Journal of the Royal Meteorological Society, v. 134, p. 2029-2050.

Parrish, M. A., H. Moradkhani, and C. M. DeChant, 2012, Toward reduction of model uncertainty: Integration of Bayesian model averaging and data assimilation: Water Resources Research, v. 48, W03519, doi:10.1029/2011WR011116.

Raftery, A. E., T. Gneiting, F. Balabdaoui, and M. Polakowski, 2005, Using Bayesian Model Averaging to Calibrate Forecast Ensembles: Monthly Weather Review, v. 133, p. 1155-1174.

Rayner, S., D. Lach, and H. Ingram, 2005, Weather Forecasts are for Wimps: Why Water Resource Managers Do Not Use Climate Forecasts: Climatic Change, v. 69, p. 197227.

Robertson, D. E., D. L. Shrestha, and Q. J. Wang, 2013, Post processing rainfall forecasts from numerical weather prediction models for short term streamflow forecasting: Hydrology and Earth System Sciences Discussions, v. 10, p. 6765-6806.

Saha, S., S. Moorthi, H.-L. Pan, X. Wu, J. Wang, S. Nadiga, P. Tripp, R. Kistler, J. Woollen, and D. Behringer, 2010, The NCEP climate forecast system reanalysis: Bulletin of the American Meteorological Society, v. 91, p. 1015-1057.

Salvadori, G., and C. De Michele, 2010, Multivariate multiparameter extreme value models and return periods: A copula approach: Water resources research, v. 46.

Schaake, J., J. Demargne, R. Hartman, M. Mullusky, E. Welles, L. Wu, H. Herr, X. Fan, and D. J. Seo, 2007, Precipitation and temperature ensemble forecasts from singlevalue forecasts: Hydrology and Earth System Sciences Discussions Discussions, v. 4, p. 655-717.

Schefzik, R., T. L. Thorarinsdottir, and T. Gneiting, 2013, Uncertainty quantification in complex simulation models using ensemble copula coupling: Statistical Science, v. 28 , p. 616-640. 
Seo, D. J., S. Perica, E. Welles, and J. C. Schaake, 2000, Simulation of precipitation fields from probabilistic quantitative precipitation forecast: Journal of Hydrology, v. 239, p. 203-229.

Sharma, D., A. D. Gupta, and M. S. Babel, 2007, Spatial disaggregation of bias-corrected GCM precipitation for improved hydrologic simulation: Ping River Basin, Thailand: Hydrology and Earth System Sciences Discussions, v. 11, p. 1373-1390.

Sklar, M., 1959, Fonctions de répartition à n dimensions et leurs marges, Université Paris 8.

Stephens, M. A., 1974, EDF statistics for goodness of fit and some comparisons: Journal of the American statistical Association, v. 69, p. 730-737.

Tao, Y., Q. Duan, A. Ye, W. Gong, Z. Di, M. Xiao, and K. Hsu, 2014, An evaluation of post-processed TIGGE multimodel ensemble precipitation forecast in the Huai river basin: Journal of Hydrology, v. 519, Part D, p. 2890-2905.

Wang, Q. J., and D. E. Robertson, 2011, Multisite probabilistic forecasting of seasonal flows for streams with zero value occurrences: Water Resources Research, v. 47, p. W02546.

Wang, Q. J., D. E. Robertson, and F. H. S. Chiew, 2009, A Bayesian joint probability modeling approach for seasonal forecasting of streamflows at multiple sites: Water Resources Research, v. 45, p. W05407.

Wood, A. W., L. R. Leung, V. Sridhar, and D. P. Lettenmaier, 2004, Hydrologic implications of dynamical and statistical approaches to downscaling climate model outputs: Climatic change, v. 62, p. 189-216.

Wood, A. W., and J. C. Schaake, 2008, Correcting errors in streamflow forecast ensemble mean and spread: Journal of Hydrometeorology, v. 9, p. 132-148.

Wu, L., D.-J. Seo, J. Demargne, J. D. Brown, S. Cong, and J. Schaake, 2011, Generation of ensemble precipitation forecast from single-valued quantitative precipitation forecast for hydrologic ensemble prediction: Journal of hydrology, v. 399, p. 281298.

Zhao, L., Q. Duan, J. Schaake, A. Ye, and J. Xia, 2011, A hydrologic post-processor for ensemble streamflow predictions: Advances in Geosciences, v. 29, p. 51-59. 